\title{
REALIZABILITY ALGEBRAS: A PROGRAM TO WELL ORDER $\mathbb{R}$
}

\author{
JEAN-LOUIS KRIVINE
}

Université Paris VII, C.N.R.S.

e-mail address: krivine@pps.jussieu.fr

\begin{abstract}
The theory of classical realizability is a framework in which we can develop the proof-program correspondence. Using this framework, we show how to transform into programs the proofs in classical analysis with dependent choice and the existence of a well ordering of the real line. The principal tools are:

- The notion of realizability algebra, which is a three-sorted variant of the well known combinatory algebra of Curry.

- An adaptation of the method of forcing used in set theory to prove consistency results. Here, it is used in another way, to obtain programs associated with a well ordering of $\mathbb{R}$ and the existence of a non trivial ultrafilter on $\mathbb{N}$.
\end{abstract}

\section{INTRODUCTION}

When we want to obtain programs from mathematical proofs, the main problem is, naturally, raised by the axioms: indeed, it has been a long time since we know how to transform a proof in pure (i.e. without axioms) intuitionistic logic, even at second order [2, 7, 4].

The very first of these axioms is the excluded middle, and it seemed completely hopeless for decades. The solution, given by T. Griffin [5] in 1990, was absolutely surprising. It was an essential discovery in logic because, at this moment, it became clear that all other axioms will follow, as soon as we will work in a suitable framework.

The theory of classical realizability is such a framework: it was developed in [12, 13], where we treat the axioms of Analysis (second order arithmetic with dependent choice).

In [15], we attack a more difficult case of the general axiom of choice, which is the existence of a non trivial ultrafilter on $\mathbb{N}$; the main tool is the notion of realizability structure, in which the programs are written in $\lambda$-calculus.

In the present paper, we replace it with the notion of realizability algebra, which has many advantages: it is simpler, first order and much more practical for implementation. It is a three-sorted variant of the usual notion of combinatory algebra. Thus, the programming language is no longer the $\lambda$-calculus, but a suitable set of combinators ; remarkably enough, this is almost exactly the original set given by Curry. The $\lambda$-terms are now considered only as notations or abbreviations, very useful in fact: a $\lambda$-term is infinitely more readable than

1998 ACM Subject Classification: F.4.1.

Key words and phrases: Curry-Howard correspondence, combinatory logic, lambda-calculus, axiom of choice. 
its translation into a sequence of combinators. The translation used here is new, as far as I know ; its fundamental property is given in theorem 1.2.

The aim of this paper is to show how to transform into programs, the classical proofs which use dependent choice and:

i) the existence of a non trivial ultrafilter on $\mathbb{N}$;

ii) the existence of a well ordering on $\mathbb{R}$.

Of course, (ii) implies (i) but the method used for (i) is interesting, because it can give simpler programs. This is an important point, because a new problem is appearing now, an important and very difficult problem: to understand the programs we obtain in this way, that is to explain their behavior. A fascinating, but probably long work.

The logical frame is given by classical second order logic, in other words the (first order) theory of the comprehension scheme. However, since we use a binary membership relation on individuals, we work, in reality, in at least third order logic. Moreover, this is indispensable since, although the axiom of dependent choice on $\mathbb{R}$ can be expressed as a second order scheme, axioms (i) and (ii) cannot be expressed in this way.

By using the method expounded in [11, we can obtain the same results in ZF.

It seems clear to me that, by developing the technology of classical realizability, we shall be able to treat all "natural" axioms introduced in set theory. It is already done for the continuum hypothesis, which will be the topic of a forthcoming paper. In my opinion, the axiom of choice and the generalized continuum hypothesis in ZF do not pose serious issues, except this: it will be necessary to use the proper class forcing of Easton [3] inside the realizability model, and it will probably be very painful.

A very interesting open problem is posed by axioms such as the existence of measurable cardinals or the determination axiom.

But the most important open problem is to understand what all these programs do and, in this way, to be able to execute them. I believe that big surprises are waiting for us here.

Indeed, when we realize usual axioms of mathematics, we need to introduce, one after the other, the very standard tools in system programming: for the law of Peirce, these are continuations (particularly useful for exceptions) ; for the axiom of dependent choice, these are the clock and the process numbering; for the ultrafilter axiom and the well ordering of $\mathbb{R}$, these are no less than read and write instructions on a global memory, in other words assignment.

It seems reasonable to conjecture that such tools are introduced for some worthwhile purpose, and therefore that the very complex programs we obtain by means of this formalization work, perform interesting and useful tasks. The question is: which ones ?

\section{Remark.}

The problem of obtaining a program from a proof which uses a given axiom, must be set correctly from the point of view of computer science. As an example, consider a proof of a theorem of arithmetic, which uses a well ordering of $\mathcal{P}(\mathbb{N})$ : if you restrict this proof to the class of constructible sets, you easily get a new proof of the same theorem, which does not use this well ordering any more. Thus, it looks like you simply have to transform this new proof into a program.

But this program would be extracted from a proof which is deeply different from (and dramatically more complicated than) the original one. Moreover, with this method, it is impossible to associate a program with the well ordering axiom itself. From the point of view of computer science, this is an unacceptable lack of modularity: since we cannot put the well ordering axiom in a program library, we need to undertake again the programming work with each new proof. 
With the method which is explained below, we only use the $\lambda$-term extracted from the original proof. Therefore, this term contains an unknown instruction for the well ordering axiom on $\mathcal{P}(\mathbb{N})$, which is not yet implemented. Then, by means of a suitable compilation, we transform this term into a true program which realizes the initial theorem.

As a corollary of this technology, we obtain a program which is associated with the well ordering axiom, which we can put in a library for later use.

\section{Realizability AlgeBras}

A realizability algebra is composed of three sets: $\boldsymbol{\Lambda}$ (the set of terms), $\boldsymbol{\Pi}$ (the set of stacks), $\boldsymbol{\Lambda} \star \boldsymbol{\Pi}$ (the set of processes) with the following operations:

$(\xi, \eta) \mapsto(\xi) \eta$ from $\boldsymbol{\Lambda}^{2}$ into $\boldsymbol{\Lambda}$ (application) ;

$(\xi, \pi) \mapsto \xi \cdot \pi$ from $\boldsymbol{\Lambda} \times \boldsymbol{\Pi}$ into $\boldsymbol{\Pi}($ push $) ;$

$(\xi, \pi) \mapsto \xi \star \pi$ from $\boldsymbol{\Lambda} \times \boldsymbol{\Pi}$ into $\boldsymbol{\Lambda} \star \boldsymbol{\Pi}$ (process) ;

$\pi \mapsto \mathrm{k}_{\pi}$ from $\boldsymbol{\Pi}$ into $\boldsymbol{\Lambda}$ (continuation).

We have, in $\boldsymbol{\Lambda}$, the distinguished elements $B, C, E, I, K, W, \mathrm{cc}$, called elementary combinators or instructions.

Notation. The term $\left(\ldots\left(\left((\xi) \eta_{1}\right) \eta_{2}\right) \ldots\right) \eta_{n}$ will be also denoted by $(\xi) \eta_{1} \eta_{2} \ldots \eta_{n}$ or even $\xi \eta_{1} \eta_{2} \ldots \eta_{n}$. For example: $\xi \eta \zeta=(\xi) \eta \zeta=(\xi \eta) \zeta=((\xi) \eta) \zeta$.

We define on $\boldsymbol{\Lambda} \star \boldsymbol{\Pi}$ a preorder relation, denoted by $\succ$. It is the least reflexive and transitive relation such that we have, for any $\xi, \eta, \zeta \in \boldsymbol{\Lambda}$ and $\pi, \varpi \in \mathbf{\Pi}$ :

$(\xi) \eta \star \pi \succ \xi \star \eta \cdot \pi$.

$I \star \xi \cdot \pi \succ \xi \star \pi$.

$K \star \xi \cdot \eta \cdot \pi \succ \xi \star \pi$.

$E \star \xi \cdot \eta \cdot \pi \succ(\xi) \eta \star \pi$.

$W \star \xi \cdot \eta \cdot \pi \succ \xi \star \eta \cdot \eta \cdot \pi$.

$C \star \xi \cdot \eta \cdot \zeta \cdot \pi \succ \xi \star \zeta \cdot \eta \cdot \pi$.

$B \star \xi \cdot \eta \cdot \zeta \cdot \pi \succ(\xi)(\eta) \zeta \star \pi$.

$\mathrm{cC} \star \xi \cdot \pi \succ \xi \star \mathrm{k}_{\pi} \cdot \pi$.

$\mathrm{k}_{\pi} \star \xi \cdot \varpi \succ \xi \star \pi$.

Finally, we are given a subset $\Perp$ of $\boldsymbol{\Lambda} \star \boldsymbol{\Pi}$ which is a terminal segment for this preorder, which means that: $p \in \Perp, p^{\prime} \succ p \Rightarrow p^{\prime} \in \Perp \Perp \Perp$.

In other words, we ask that $\Perp$ be such that:

$(\xi) \eta \star \pi \notin \Perp \Perp \xi \star \eta \cdot \pi \notin \Perp$.

$I \star \xi \cdot \pi \notin \Perp \Rightarrow \xi \star \pi \notin \Perp \Perp$.

$K \star \xi \cdot \eta \cdot \pi \notin \Perp \Rightarrow \xi \star \pi \notin \Perp$.

$E \star \xi \cdot \eta \cdot \pi \notin \Perp \Perp(\xi) \eta \star \pi \notin \Perp \Perp$.

$W \star \xi \cdot \eta \cdot \pi \notin \Perp \Rightarrow \xi \star \eta \cdot \eta \cdot \pi \notin \Perp \Perp$.

$C \star \xi \cdot \eta \cdot \zeta \cdot \pi \notin \Perp \# \xi \star \zeta \cdot \eta \cdot \pi \notin \Perp \Perp$.

$B \star \xi \cdot \eta \cdot \zeta \cdot \pi \notin \Perp \Rightarrow(\xi)(\eta) \zeta \star \pi \notin \Perp \Perp$.

$\mathrm{CC} \star \xi \cdot \pi \notin \Perp \Perp \xi \star \mathrm{k}_{\pi} \cdot \pi \notin \Perp \Perp$.

$\mathrm{k}_{\pi} \star \xi \cdot \varpi \notin \Perp \Rightarrow \xi \star \pi \notin \Perp$. 
c-terms and $\lambda$-terms. We call c-term a term which is built with variables, the elementary combinators $B, C, E, I, K, W, \mathrm{cc}$ and the application (binary function). A c-term is called closed if it contains no variable ; it will then also be called proof-like ; a proof-like term has a value in $\boldsymbol{\Lambda}$.

Given a C-term $t$ and a variable $x$, we define inductively on $t$, a new C-term denoted by $\lambda x t$. To this aim, we apply the first possible case in the following list:

1. $\lambda x t=(K) t$ if $t$ does not contain $x$.

2. $\lambda x x=I$.

3. $\lambda x t u=(C \lambda x(E) t) u$ if $u$ does not contain $x$.

4. $\lambda x t x=(E) t$ if $t$ does not contain $x$.

5. $\lambda x t x=(W) \lambda x(E) t$ (if $t$ contains $x)$.

6. $\lambda x(t)(u) v=\lambda x(B) t u v$ (if $u v$ contains $x$ ).

We easily see that this rewriting is finite, for any given c-term $t$ : indeed, during the rewriting, no combinator is introduced inside $t$, but only in front of it. Moreover, the only changes in $t$ are: moving parentheses and erasing occurrences of $x$. Now, rules 1 to 5 strictly decrease the part of $t$ which remains under $\lambda x$, and rule 6 can be applied consecutively only finitely many times.

The $\lambda$-terms are defined as usual. But, in this paper, we consider $\lambda$-terms only as a notation for particular c-terms, by means of the above translation. This notation is essential, because almost every c-term we shall use, will be given as a $\lambda$-term. Theorem 1.2 gives the fundamental property of this translation.

Remark. We cannot use the well known $K S$-translation of $\lambda$-calculus, because it does not satisfy Theorem 1.2 ,

Lemma 1.1. If $t$ is a $\mathbf{c}$-term with the only variables $x, y_{1}, \ldots, y_{n}$, and if $\xi, \eta_{1}, \ldots, \eta_{n} \in \mathbf{\Lambda}$, then: $(\lambda x t)\left[\eta_{1} / y_{1}, \ldots, \eta_{n} / y_{n}\right] \star \xi \bullet \pi \succ t\left[\xi / x, \eta_{1} / y_{1}, \ldots, \eta_{n} / y_{n}\right] \star \pi$.

Proof. To lighten the notation, let us put $u^{*}=u\left[\eta_{1} / y_{1}, \ldots, \eta_{n} / y_{n}\right]$ for each c-term $u$; thus, we have:

$u^{*}[\xi / x]=u\left[\xi / x, \eta_{1} / y_{1}, \ldots, \eta_{n} / y_{n}\right]$.

The proof is done by induction on the number of rules 1 to 6 used to translate the term $\lambda x t$. Consider the rule used first.

If it is rule 1 , then we have $(\lambda x t)^{*} \star \xi \cdot \pi \equiv(K) t^{*} \star \xi \cdot \pi \succ t^{*} \star \pi$ $\equiv t\left[\xi / x, \eta_{1} / y_{1}, \ldots, \eta_{n} / y_{n}\right] \star \pi$ since $x$ is not in $t$.

If it is rule 2 , we have $t=x$ and $(\lambda x t)^{*} \star \xi \bullet \pi \equiv I \star \xi \bullet \pi \succ \xi \star \pi \equiv t\left[\xi / x, \eta_{1} / y_{1}, \ldots, \eta_{n} / y_{n}\right] \star \pi$. If it is rule 3, we have $t=u v$ and $(\lambda x t)^{*} \star \xi \cdot \pi \equiv(C \lambda x(E) u)^{*} v^{*} \star \xi \cdot \pi$ $\succ C \star(\lambda x(E) u)^{*} \cdot v^{*} \cdot \xi \cdot \pi \succ(\lambda x(E) u)^{*} \star \xi \cdot v^{*} \cdot \pi \succ(E) u^{*}[\xi / x] \star v^{*} \cdot \pi$ (by induction hypothesis $) \succ E \star u^{*}[\xi / x] \cdot v^{*} \cdot \pi \succ\left(u^{*}[\xi / x]\right) v^{*} \star \pi \equiv t\left[\xi / x, \eta_{1} / y_{1}, \ldots, \eta_{n} / y_{n}\right] \star \pi$ since $x$ is not in $v$.

If it is rule 4, we have $t=u x$ and $(\lambda x t)^{*} \star \xi \cdot \pi \equiv(E) u^{*} \star \xi \cdot \pi \succ E \star u^{*} \cdot \xi \bullet \pi \succ u^{*} \xi \star \pi$ $\equiv t\left[\xi / x, \eta_{1} / y_{1}, \ldots, \eta_{n} / y_{n}\right] \star \pi$ since $u$ does not contain $x$.

If it is rule 5, we have $t=u x$ and $(\lambda x t)^{*} \star \xi \bullet \pi \equiv(W \lambda x(E) u)^{*} \star \xi \cdot \pi \succ W \star(\lambda x(E) u)^{*} \cdot \xi \cdot \pi$ $\succ(\lambda x(E) u)^{*} \star \xi \cdot \xi \cdot \pi \succ(E) u^{*}[\xi / x] \star \xi \cdot \pi$ (by induction hypothesis)

$\succ E \star u^{*}[\xi / x] \cdot \xi \cdot \pi \succ\left(u^{*}[\xi / x]\right) \xi \star \pi \equiv t\left[\xi / x, \eta_{1} / y_{1}, \ldots, \eta_{n} / y_{n}\right] \star \pi$.

If it is rule 6 , we have $t=(u)(v) w$ and $(\lambda x t)^{*} \star \xi \cdot \pi \equiv(\lambda x(B) u v w)^{*} \star \xi \cdot \pi$ $\succ(B) u^{*}[\xi / x] v^{*}[\xi / x] w^{*}[\xi / x] \star \pi$ (by induction hypothesis) 
$\succ B \star u^{*}[\xi / x] \cdot v^{*}[\xi / x] \cdot w^{*}[\xi / x] \cdot \pi \succ\left(u^{*}[\xi / x]\right)\left(v^{*}[\xi / x]\right) w^{*}[\xi / x] \star \pi$

$\equiv t\left[\xi / x, \eta_{1} / y_{1}, \ldots, \eta_{n} / y_{n}\right] \star \pi$.

Theorem 1.2. If $t$ is a c-term with the only variables $x_{1}, \ldots, x_{n}$, and if $\xi_{1}, \ldots, \xi_{n} \in \mathbf{\Lambda}$, then $\lambda x_{1} \ldots \lambda x_{n} t \star \xi_{1} \cdot \ldots \cdot \xi_{n} \cdot \pi \succ t\left[\xi_{1} / x_{1}, \ldots, \xi_{n} / x_{n}\right] \star \pi$.

Proof. By induction on $n$; the case $n=0$ is trivial.

We have $\lambda x_{1} \ldots \lambda x_{n-1} \lambda x_{n} t \star \xi_{1} \cdot \ldots \cdot \xi_{n-1} \cdot \xi_{n} \bullet \pi \succ\left(\lambda x_{n} t\right)\left[\xi_{1} / x_{1}, \ldots, \xi_{n-1} / x_{n-1}\right] \star \xi_{n} \bullet \pi$

(by induction hypothesis) $\succ t\left[\xi_{1} / x_{1}, \ldots, \xi_{n-1} / x_{n-1}, \xi_{n} / x_{n}\right] \star \pi$ by lemma 1.1.

Natural deduction. Before giving the formal language that we shall use, it is perhaps useful to describe informally the structures (models) we have in mind. They are second order structures, with two types of objects: individuals also called conditions and predicates (of various arity). Since we remain at an intuitive level, we start with a full model which we call the ground model. Such a model consists of:

- an infinite set $P$ (the set of individuals or conditions).

- the set of $k$-ary predicates is $\mathcal{P}\left(P^{k}\right)$ (full model).

- some functions from $P^{k}$ into $P$.

In particular, there is an individual 0 and a bijective function $s: P \rightarrow(P \backslash\{0\})$. This enables us to define the set of integers $\mathbb{N}$ as the least set which contains 0 and which is closed for $s$.

There is also a particular condition denoted by $\mathbf{1}$ and an application denoted by $\wedge$ from $P^{2}$ into $P$.

- some relations (fixed predicates) on $P$. In particular, we have the equality relation on individuals and the subset $\mathrm{C}$ of non trivial conditions.

$\mathrm{C}[p \wedge q]$ reads as: " $p$ and $q$ are two compatible conditions".

We now come to the formal language, in order to write formulas and proofs about such structures. It consists of:

- individual variables or variables of conditions called $x, y, \ldots$ or $p, q, \ldots$

- predicate variables or second order variables $X, Y, \ldots$; each predicate variable has an arity which is in $\mathbb{N}$.

- function symbols on individuals $f, g, \ldots$; each one has an arity which is in $\mathbb{N}$.

In particular, there is a function symbol of arity $k$ for each recursive function $f: \mathbb{N}^{k} \rightarrow \mathbb{N}$. This symbol will also be written as $f$.

There is also a constant symbol $\mathbf{1}$ (which represents the greatest condition) and a binary function symbol $\wedge$ (which represents the inf of two conditions).

The terms are built in the usual way with variables and function symbols.

The atomic formulas are the expressions $X\left(t_{1}, \ldots, t_{n}\right)$, where $X$ is an $n$-ary predicate variable, and $t_{1}, \ldots, t_{n}$ are terms.

Formulas are built as usual, from atomic formulas, with the only logical symbols $\rightarrow, \forall$ :

- each atomic formula is a formula;

- if $A, B$ are formulas, then $A \rightarrow B$ is a formula ;

- if $A$ is a formula, then $\forall x A$ and $\forall X A$ are formulas.

\section{Notations.}

The formula $A_{1} \rightarrow\left(A_{2} \rightarrow\left(\ldots\left(A_{n} \rightarrow B\right) \ldots\right)\right.$ will be written $A_{1}, A_{2}, \ldots, A_{n} \rightarrow B$.

The usual logical symbols are defined as follows: 
( $X$ is a predicate variable of arity 0 , also called propositional variable) $\perp \equiv \forall X X ; \neg A \equiv A \rightarrow \perp ; A \vee B \equiv(A \rightarrow \perp),(B \rightarrow \perp) \rightarrow \perp ; A \wedge B \equiv(A, B \rightarrow \perp) \rightarrow \perp$; $\exists \mathbf{y} F \equiv \forall \mathbf{y}(F \rightarrow \perp) \rightarrow \perp$ (where $\mathbf{y}$ is an individual or predicate variable). More generally, we shall write $\exists \mathbf{y}\left\{F_{1}, \ldots, F_{k}\right\}$ for $\forall \mathbf{y}\left(F_{1}, \ldots, F_{k} \rightarrow \perp\right) \rightarrow \perp$. We shall sometimes write $\vec{F}$ for a finite sequence of formulas $F_{1}, \ldots, F_{k}$. Then, we shall also write $\exists \mathbf{y}\{\vec{F}\}$ and $\forall \mathbf{y}(\vec{F} \rightarrow \perp) \rightarrow \perp$. $x=y$ is the formula $\forall Z(Z x \rightarrow Z y)$, where $Z$ is a unary predicate variable.

The rules of natural deduction are the following (the $A_{i}$ 's are formulas, the $x_{i}$ 's are variables of c-terms, $t, u$ are c-terms):

1. $x_{1}: A_{1}, \ldots, x_{n}: A_{n} \vdash x_{i}: A_{i}$.

2. $x_{1}: A_{1}, \ldots, x_{n}: A_{n} \vdash t: A \rightarrow B, \quad x_{1}: A_{1}, \ldots, x_{n}: A_{n} \vdash u: A \Rightarrow x_{1}: A_{1}, \ldots, x_{n}:$ $A_{n} \vdash t u: B$.

3. $x_{1}: A_{1}, \ldots, x_{n}: A_{n}, x: A \vdash t: B \Rightarrow x_{1}: A_{1}, \ldots, x_{n}: A_{n} \vdash \lambda x t: A \rightarrow B$.

4. $x_{1}: A_{1}, \ldots, x_{n}: A_{n} \vdash t: A \quad \Rightarrow \quad x_{1}: A_{1}, \ldots, x_{n}: A_{n} \vdash t: \forall \mathbf{x} A$ for every variable $\mathbf{x}$ (individual or predicate) which does not appear in $A_{1}, \ldots, A_{n}$.

5. $x_{1}: A_{1}, \ldots, x_{n}: A_{n} \vdash t: \forall x A \Rightarrow x_{1}: A_{1}, \ldots, x_{n}: A_{n} \vdash t: A[\tau / x]$ where $x$ is an individual variable and $\tau$ is a term.

6. $x_{1}: A_{1}, \ldots, x_{n}: A_{n} \vdash t: \forall X A \Rightarrow x_{1}: A_{1}, \ldots, x_{n}: A_{n} \vdash t: A\left[F / X y_{1} \ldots y_{k}\right]$ where $X$ is a predicate variable of arity $k$ and $F$ an arbitrary formula.

Remark.

In the notation $A\left[F / X y_{1} \ldots y_{k}\right]$, the variables $y_{1}, \ldots, y_{k}$ are bound. A more usual notation is: $A\left[\lambda y_{1} \ldots \lambda y_{k} F / X\right]$. I prefer this one, to avoid confusion with the $\lambda$ defined for c-terms.

Realizability. Given a realizability algebra $\mathcal{A}=(\boldsymbol{\Lambda}, \boldsymbol{\Pi}, \boldsymbol{\Lambda} \star \boldsymbol{\Pi}, \Perp)$, a $\mathcal{A}$-model $\mathcal{M}$ consists of the following data:

- An infinite set $P$ which is the domain of variation of individual variables.

- The domain of variation of $k$-ary predicate variables is $\mathcal{P}(\boldsymbol{\Pi})^{P^{k}}$.

- We associate with each $k$-ary function symbol $f$, a function from $P^{k}$ into $P$, denoted by $\bar{f}$ or even $f$ if there is no ambiguity.

In particular, there is a distinguished element 0 in $P$ and a function $s: P \rightarrow P$ (which is the interpretation of the symbol $s$ ). We suppose that $s$ is a bijection from $P$ onto $P \backslash\{0\}$. Then, we can identify $s^{n} 0 \in P$ with the integer $n$, and therefore, we have $\mathbb{N} \subset P$.

Each recursive function $f: \mathbb{N}^{k} \rightarrow \mathbb{N}$ is, by hypothesis, a function symbol. Of course, we assume that its interpretation $\bar{f}: P^{k} \rightarrow P$ takes the same values as $f$ on $\mathbb{N}^{k}$.

Finally, we have also a condition $1 \in P$ and a binary function $\wedge$ from $P^{2}$ into $P$.

A closed term (resp. a closed formula) with parameters in the model $\mathcal{M}$ is, by definition, a term (resp. a formula) in which all free occurrences of each variable have been replaced with a parameter, i.e. an object of the same type in the model $\mathcal{M}$ : a condition for an individual variable, an application from $P^{k}$ into $\mathcal{P}(\boldsymbol{\Pi})$ for a $k$-ary predicate variable.

Each closed term $t$, with parameters in $\mathcal{M}$ has a value $\bar{t} \in P$.

An interpretation $\mathcal{I}$ is an application which associates an individual (condition) with each individual variable and a parameter of arity $k$ with each second order $k$-ary variable.

$\mathcal{I}[x \leftarrow p]$ (resp. $\mathcal{I}[X \leftarrow \mathcal{X}]$ ) is, by definition, the interpretation obtained by changing, in $\mathcal{I}$, the value of the variable $x$ (resp. $X$ ) and giving to it the value $p \in P\left(\right.$ resp. $\left.\mathcal{X} \in \mathcal{P}(\boldsymbol{\Pi})^{P^{k}}\right)$. 
For each formula $F$ (resp. term $t$ ), we denote by $F^{\mathcal{I}}$ (resp. $t^{\mathcal{I}}$ ) the closed formula (resp. term) with parameters obtained by replacing each free variable with the value given by $\mathcal{I}$. For each closed formula $F^{\mathcal{I}}$ with parameters in $\mathcal{M}$, we define two truth values: $\left\|F^{\mathcal{I}}\right\| \subset \boldsymbol{\Pi}$ and $\left|F^{\mathcal{I}}\right| \subset \boldsymbol{\Lambda}$.

$\left|F^{\mathcal{I}}\right|$ is defined as follows: $\xi \in\left|F^{\mathcal{I}}\right| \Leftrightarrow\left(\forall \pi \in\left\|F^{\mathcal{I}}\right\|\right) \xi \star \pi \in \Perp$.

$\left\|F^{\mathcal{I}}\right\|$ is defined by recurrence on $F$ :

- $F$ is atomic: then $F^{\mathcal{I}}$ has the form $\mathcal{X}\left(t_{1}, \ldots, t_{k}\right)$ where $\mathcal{X}: P^{k} \rightarrow \mathcal{P}(\boldsymbol{\Pi})$ and the $t_{i}$ 's are closed terms with parameters in $\mathcal{M}$. We set $\left\|\mathcal{X}\left(t_{1}, \ldots, t_{k}\right)\right\|=\mathcal{X}\left(\bar{t}_{1}, \ldots, \bar{t}_{k}\right)$.

- $F \equiv A \rightarrow B$ : we set $\left\|F^{\mathcal{I}}\right\|=\left\{\xi \cdot \pi ; \xi \in\left|A^{\mathcal{I}}\right|, \pi \in\left\|B^{\mathcal{I}}\right\|\right\}$.

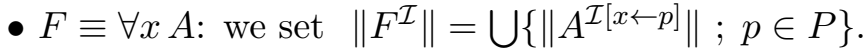

- $F \equiv \forall X A$ : we set $\left\|F^{\mathcal{I}}\right\|=\bigcup\left\{\left\|A^{\mathcal{I}[X \leftarrow \mathcal{X}]}\right\| ; \mathcal{X} \in \mathcal{P}(\boldsymbol{\Pi})^{P^{k}}\right\}$ if $X$ is a $k$-ary predicate variable.

Notation. We shall write $\xi \Vdash F$ for $\xi \in|F|$.

Theorem 1.3 (Adequacy lemma).

If $x_{1}: A_{1}, \ldots, x_{k}: A_{k} \vdash t: A$ and if $\xi_{1} \Vdash A_{1}^{\mathcal{I}}, \ldots, \xi_{k} \Vdash A_{k}^{\mathcal{I}}$, where $\mathcal{I}$ is an interpretation, then $t\left[\xi_{1} / x_{1}, \ldots, \xi_{k} / x_{k}\right] \Vdash A^{\mathcal{I}}$.

In particular, if $A$ is closed and if $\vdash t: A$, then $t \Vdash A$.

Proof. By recurrence on the length of the derivation of $x_{1}: A_{1}, \ldots, x_{n}: A_{n} \vdash t: A$.

We consider the last used rule.

1. We have $t=x_{i}, A \equiv A_{i}$. Now, we have assumed that $\xi_{i} \Vdash A_{i}^{\mathcal{I}}$; and it is the desired result.

2. We have $t=u v$ and we already obtained:

$x_{1}: A_{1}, \ldots, x_{k}: A_{k} \vdash u: B \rightarrow A$ and $x_{1}: A_{1}, \ldots, x_{k}: A_{k} \vdash v: B$.

Given $\pi \in\left\|A^{\mathcal{I}}\right\|$, we must show $(u v)\left[\xi_{1} / x_{1}, \ldots, \xi_{k} / x_{k}\right] \star \pi \in \Perp \Perp$.

By hypothesis on $\Perp$, it suffices to show $u\left[\xi_{1} / x_{1}, \ldots, \xi_{k} / x_{k}\right] \star v\left[\xi_{1} / x_{1}, \ldots, \xi_{k} / x_{k}\right] \bullet \pi \in \Perp$.

By the induction hypothesis, we have $v\left[\xi_{1} / x_{1}, \ldots, \xi_{k} / x_{k}\right] \Vdash B^{\mathcal{I}}$ and therefore:

$v\left[\xi_{1} / x_{1}, \ldots, \xi_{k} / x_{k}\right] \cdot \pi \in\left\|B^{\mathcal{I}} \rightarrow A^{\mathcal{I}}\right\|$.

But, by the induction hypothesis, we have also $u\left[\xi_{1} / x_{1}, \ldots, \xi_{k} / x_{k}\right] \| B^{\mathcal{I}} \rightarrow A^{\mathcal{I}}$, hence the result.

3. We have $A=B \rightarrow C, t=\lambda x u$. We must show $\lambda x u\left[\xi_{1} / x_{1}, \ldots, \xi_{k} / x_{k}\right] \Vdash B^{\mathcal{I}} \rightarrow C^{\mathcal{I}}$;

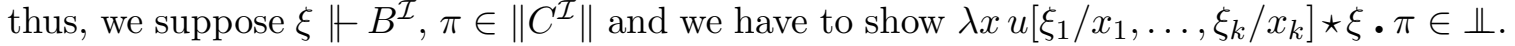
By hypothesis on $\Perp$ and lemma 1.1, it suffices to show $u\left[\xi / x, \xi_{1} / x_{1}, \ldots, \xi_{k} / x_{k}\right] \star \pi \in \Perp$.

This follows from the induction hypothesis applied to $x_{1}: A_{1}, \ldots, x_{n}: A_{n}, x: B \vdash u: C$.

4. We have $A \equiv \forall X B$, and $X$ is not free in $A_{1}, \ldots, A_{n}$. We must show:

$t\left[\xi_{1} / x_{1}, \ldots, \xi_{k} / x_{k}\right] \Vdash(\forall X B)^{\mathcal{I}}$, i.e. $t\left[\xi_{1} / x_{1}, \ldots, \xi_{k} / x_{k}\right] \Vdash B^{\mathcal{J}}$ with $\mathcal{J}=\mathcal{I}[X \leftarrow \mathcal{X}]$. But, by hypothesis, $\xi_{i} \Vdash A_{i}^{\mathcal{I}}$ therefore $\xi_{i} \Vdash A_{i}^{\mathcal{J}}$ : indeed, since $X$ is not free in $A_{i}$, we have: $\left\|A_{i}^{\mathcal{I}}\right\|=\left\|A_{i}^{\mathcal{J}}\right\|$. Then, the induction hypothesis gives the result.

6. We have $A=B\left[F / X y_{1} \ldots y_{n}\right]$ and we must show:

$t\left[\xi_{1} / x_{1}, \ldots, \xi_{k} / x_{k}\right] \Vdash B\left[F / X y_{1} \ldots y_{n}\right]^{\mathcal{I}}$ assuming that $t\left[\xi_{1} / x_{1}, \ldots, \xi_{k} / x_{k}\right] \Vdash(\forall X B)^{\mathcal{I}}$. This follows from lemma 1.4 below.

Lemma 1.4. $\left\|B\left[F / X y_{1} \ldots y_{n}\right]^{\mathcal{I}}\right\|=\left\|B^{\mathcal{I}[X \leftarrow \mathcal{X}]}\right\|$ where $\mathcal{X}: P^{n} \rightarrow \mathcal{P}(\boldsymbol{\Pi})$ is defined by: $\mathcal{X}\left(p_{1}, \ldots, p_{n}\right)=\left\|F^{\mathcal{I}\left[y_{1} \leftarrow p_{1}, \ldots, y_{n} \leftarrow p_{n}\right]}\right\|$.

Proof. The proof is by induction on $B$. That is trivial if $X$ is not free in $B$. Indeed, the only non trivial case of the induction is $B=\forall Y C$; and then, we have $Y \neq X$ and: 
$\left\|B\left[F / X y_{1} \ldots y_{n}\right]^{\mathcal{I}}\right\|=\left\|\left(\forall Y C\left[F / X y_{1} \ldots y_{n}\right]\right)^{\mathcal{I}}\right\|=\bigcup_{\mathcal{Y}}\left\|C\left[F / X y_{1} \ldots y_{n}\right]^{\mathcal{I}[Y \leftarrow \mathcal{Y}]}\right\|$.

By induction hypothesis, this gives $\bigcup_{\mathcal{Y}}\left\|C^{\mathcal{I}[Y \leftarrow \mathcal{Y}][X \leftarrow \mathcal{X}]}\right\|$, that is $\bigcup_{\mathcal{Y}}\left\|C^{\mathcal{I}[X \leftarrow \mathcal{X}][Y \leftarrow \mathcal{Y}]}\right\|$ i.e. $\left\|(\forall Y C)^{\mathcal{I}[X \leftarrow \mathcal{X}]}\right\|$.

Lemma 1.5. Let $\mathcal{X}, Y \subset \Pi$ be truth values. If $\pi \in \mathcal{X}$, then $k_{\pi} \Vdash \mathcal{X} \rightarrow \mathcal{Y}$.

Proof. Suppose $\xi \Vdash \mathcal{X}$ and $\rho \in \mathcal{Y}$; we must show $\mathrm{k}_{\pi} \star \xi \bullet \rho \in \Perp$, that is $\xi \star \pi \in \Perp$, which is clear.

Proposition 1.6 (Law of Peirce). cc $\Vdash \forall X \forall Y(((X \rightarrow Y) \rightarrow X) \rightarrow X)$.

Proof. We want to show that $\mathrm{cc} \Vdash((\mathcal{X} \rightarrow \mathcal{Y}) \rightarrow \mathcal{X}) \rightarrow \mathcal{X}$. Thus, we take $\xi \Vdash(\mathcal{X} \rightarrow \mathcal{Y}) \rightarrow$ $\mathcal{X}$ and $\pi \in \mathcal{X}$; we must show that $\mathrm{cc} \star \xi \cdot \pi \in \Perp$, that is $\xi \star \mathrm{k}_{\pi} \cdot \pi \in \Perp$. By hypothesis on $\xi$ and $\pi$, it is sufficient to show that $\mathrm{k}_{\pi} \Vdash \mathcal{X} \rightarrow \mathcal{Y}$, which results from lemma 1.5.

\section{Proposition 1.7.}

i) If $\xi \Vdash A \rightarrow B$, then $\forall \eta(\eta \Vdash A \Rightarrow \xi \eta \Vdash B)$.

ii) If $\forall \eta(\eta \Vdash A \Rightarrow \xi \eta \Vdash B)$, then $(E) \xi \Vdash A \rightarrow B$.

Proof.

i) From $\xi \eta \star \pi \succ \xi \star \eta \cdot \pi$.

ii) From $(E) \xi \star \eta \cdot \pi \succ \xi \eta \star \pi$.

Remark. Proposition 1.7 shows that $\xi \Vdash A \rightarrow B$ is "almost" equivalent (i.e. up to an $\eta$-expansion of $\xi)$ to $\forall \eta(\eta \Vdash A \Rightarrow \xi \eta \Vdash B)$.

Predicate symbols. In the following, we shall use extended formulas which contain predicate symbols (or predicate constants) R,S, .. on individuals. Each one has an arity, which is an integer.

In particular, we have a unary predicate symbol C (which represents the set of non trivial conditions).

We have to add some rules of construction of formulas:

- If $F$ is a formula, $\mathrm{R}$ is a $n$-ary predicate constant and $t_{1}, \ldots, t_{n}$ are terms, then $\mathrm{R}\left(t_{1}, \ldots, t_{n}\right) \rightarrow F$ and $\mathrm{R}\left(t_{1}, \ldots, t_{n}\right) \mapsto F$ are formulas.

- $T$ is an atomic formula.

In the definition of a $\mathcal{A}$-model $\mathcal{M}$, we add the following clause:

- With each relation symbol $\mathrm{R}$ of arity $n$, we associate an application, denoted by $\overline{\mathrm{R}}_{\mathcal{M}}$ or $\overline{\mathrm{R}}$, from $P^{n}$ into $\mathcal{P}(\boldsymbol{\Lambda})$. We shall also write $\left|\mathrm{R}\left(p_{1}, \ldots, p_{n}\right)\right|$, instead of $\overline{\mathrm{R}}\left(p_{1}, \ldots, p_{n}\right)$, for $p_{1}, \ldots, p_{n} \in P$.

In particular, we have an application $\overline{\mathrm{C}}: P \rightarrow \mathcal{P}(\boldsymbol{\Lambda})$, which we denote as $|\mathrm{C}[p]|$.

We define as follows the truth value in $\mathcal{M}$ of an extended formula:

$\|\top\|=\emptyset$.

$\left\|\left(\mathrm{R}\left(t_{1}, \ldots, t_{n}\right) \rightarrow F\right)^{\mathcal{I}}\right\|=\left\{t \cdot \pi ; t \in\left|\mathrm{R}\left(t_{1}^{\mathcal{I}}, \ldots, t_{n}^{\mathcal{I}}\right)\right|, \pi \in\left\|F^{\mathcal{I}}\right\|\right\}$.

$\left\|\left(\mathrm{R}\left(t_{1}, \ldots, t_{n}\right) \mapsto F\right)^{\mathcal{I}}\right\|=\left\|F^{\mathcal{I}}\right\|$ if $I \in\left|\mathrm{R}\left(t_{1}^{\mathcal{I}}, \ldots, t_{n}^{\mathcal{I}}\right)\right| ;$

$\left\|\left(\mathrm{R}\left(t_{1}, \ldots, t_{n}\right) \mapsto F\right)^{\mathcal{I}}\right\|=\emptyset$ otherwise.

Proposition 1.8.

i) $\lambda x(x) I \Vdash \forall X \forall x_{1} \ldots \forall x_{n}\left[\left(\mathrm{R}\left(x_{1}, \ldots, x_{n}\right) \rightarrow X\right) \rightarrow\left(\mathrm{R}\left(x_{1}, \ldots, x_{n}\right) \mapsto X\right)\right]$.

ii) If we have $\left|\mathrm{R}\left(p_{1}, \ldots, p_{n}\right)\right| \neq \emptyset \Rightarrow I \in\left|\mathrm{R}\left(p_{1}, \ldots, p_{n}\right)\right|$ for every $p_{1}, \ldots, p_{n} \in P$, then:

$K \Vdash \forall X \forall x_{1} \ldots \forall x_{n}\left[\left(\mathrm{R}\left(x_{1}, \ldots, x_{n}\right) \mapsto X\right) \rightarrow\left(\mathrm{R}\left(x_{1}, \ldots, x_{n}\right) \rightarrow X\right)\right]$. 
Proof. Trivial.

Remark. By means of proposition 1.8, we see that, if the application $\bar{R}: P^{n} \rightarrow \mathcal{P}(\boldsymbol{\Lambda})$ takes only the values $\{I\}$ and $\emptyset$, we can replace $\mathrm{R}\left(t_{1}, \ldots, t_{n}\right) \rightarrow F$ with $\mathrm{R}\left(t_{1}, \ldots, t_{n}\right) \mapsto F$.

We define the binary predicate $\simeq$ by putting $|p \simeq q|=\{I\}$ if $p=q$ and $|p \simeq q|=\emptyset$ if $p \neq q$.

By the above remark, we can replace $p \simeq q \rightarrow F$ with $p \simeq q \mapsto F$. Proposition 1.9 shows that we can also replace $p=q \rightarrow F$ with $p \simeq q \mapsto F$.

Notations. We shall write $p=q \mapsto F$ instead of $p \simeq q \mapsto F$. Thus, we have: $\|p=q \mapsto F\|=\|F\|$ if $p=q ;\|p=q \mapsto F\|=\emptyset$ if $p \neq q$.

We shall write $p \neq q$ for $p=q \mapsto \perp$. Thus, we have:

$\|p \neq q\|=\Pi$ if $p=q$ and $\|p \neq q\|=\emptyset$ if $p \neq q$.

Using $p=q \mapsto F$ instead of $p=q \rightarrow F$, and $p \neq q$ instead of $p=q \rightarrow \perp$, greatly simplifies the computation of the truth value of a formula which contains the symbol $=$.

\section{Proposition 1.9.}

i) $\lambda x x I \Vdash \forall X \forall x \forall y((x=y \rightarrow X) \rightarrow(x=y \mapsto X))$;

ii) $\lambda x \lambda y y x \Vdash \forall X \forall x \forall y((x=y \mapsto X), x=y \rightarrow X)$.

\section{Proof.}

i) Let $a, b \in P, \mathcal{X} \subset \boldsymbol{\Pi}, \xi \Vdash a=b \rightarrow \mathcal{X}$ and $\pi \in\|a=b \mapsto \mathcal{X}\|$.

Then, we have $a=b$, thus $I \Vdash a=b$, therefore $\xi \star I \bullet \pi \in \Perp$, thus $\lambda x x I \star \xi \bullet \pi \in \Perp$.

ii) Now let $\eta \Vdash(a=b \mapsto \mathcal{X}), \zeta \nVdash a=b$ and $\rho \in\|\mathcal{X}\|$.

We show that $\lambda x \lambda y y x \star \eta \cdot \zeta \cdot \rho \in \Perp$ in other words $\zeta \star \eta \bullet \rho \in \Perp$.

If $a=b$, then $\eta \Vdash \mathcal{X}, \zeta \Vdash \forall Y(Y \rightarrow Y)$. We have $\eta \bullet \rho \in\|\mathcal{X} \rightarrow \mathcal{X}\|$, thus $\zeta \star \eta \bullet \rho \in \mathbb{\Perp}$.

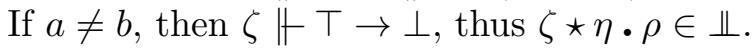

In both cases, we get the desired result.

\section{Remark.}

Let $R$ be a subset of $P^{k}$ and $1_{R}: P^{k} \rightarrow\{0,1\}$ its characteristic function, defined as follows:

$1_{R}\left(p_{1}, \ldots, p_{n}\right)=1($ resp. $=0)$ if $\left(p_{1}, \ldots, p_{n}\right) \in R\left(\right.$ resp. $\left.\left(p_{1}, \ldots, p_{n}\right) \notin R\right)$.

Let us define the predicate $R$ in the model $\mathcal{M}$ by putting:

$\left|R\left(p_{1}, \ldots, p_{n}\right)\right|=\{I\}($ resp. $=\emptyset)$ if $\left(p_{1}, \ldots, p_{n}\right) \in R\left(\operatorname{resp} .\left(p_{1}, \ldots, p_{n}\right) \notin R\right)$.

By propositions 1.8 and 1.9, we see that $R\left(x_{1}, \ldots, x_{n}\right)$ and $1_{R}\left(x_{1}, \ldots, x_{n}\right)=1$ are interchangeable. More precisely, we have: $I \Vdash \forall X \forall x_{1} \ldots \forall x_{n}\left(\left(R\left(x_{1}, \ldots, x_{n}\right) \mapsto X\right) \leftrightarrow\left(1_{R}\left(x_{1}, \ldots, x_{n}\right)=1 \mapsto X\right)\right)$.

For each formula $A\left[x_{1}, \ldots, x_{k}\right]$, we can define the $k$-ary predicate symbol $N_{A}$, by putting $\left|N_{A}\left(p_{1}, \ldots, p_{k}\right)\right|=\left\{\mathrm{k}_{\pi} ; \pi \in\left\|A\left[p_{1}, \ldots, p_{k}\right]\right\|\right\}$. Proposition 1.10 below shows that $N_{A}$ and $\neg A$ are interchangeable ; this may simplify truth value computations.

\section{Proposition 1.10.}

i) $I \Vdash \forall x_{1} \ldots \forall x_{k}\left(N_{A}\left(x_{1}, \ldots, x_{k}\right) \rightarrow \neg A\left(x_{1}, \ldots, x_{k}\right)\right)$;

ii) $\mathrm{cc} \Vdash \forall x_{1} \ldots \forall x_{k}\left(\left(N_{A}\left(x_{1}, \ldots, x_{k}\right) \rightarrow \perp\right) \rightarrow A\left(x_{1}, \ldots, x_{k}\right)\right)$.

\section{Proof.}

i) Let $p_{1}, \ldots, p_{k} \in P, \pi \in\left\|A\left(p_{1}, \ldots, p_{k}\right)\right\|, \xi \Vdash A\left(p_{1}, \ldots, p_{k}\right)$ and $\rho \in \Pi$. We must show:

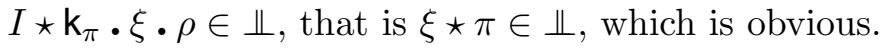

ii) Let $\eta \Vdash N_{A}\left(p_{1}, \ldots, p_{k}\right) \rightarrow \perp$ and $\pi \in\left\|A\left(p_{1}, \ldots, p_{k}\right)\right\|$. We must show:

cC $\star \eta \cdot \pi \in \Perp \Perp \Perp$, i.e. $\eta \star \mathrm{k}_{\pi} \cdot \pi \in \Perp \Perp \Perp$, which is clear, since $\mathrm{k}_{\pi} \in\left|N_{A}\left(p_{1}, \ldots, p_{k}\right)\right|$. 
Fixed point combinator.

Theorem 1.11. Let $\mathrm{Y}=A A$ with $A=\lambda a \lambda f(f)(a)$ af. Then, we have $\mathrm{Y} \star \xi \cdot \pi \succ \xi \star \mathrm{Y} \xi \bullet \pi$. Let $f: P^{2} \rightarrow P$ such that $f(x, y)=1$ is a well founded relation on $P$. Then:

i) $\mathrm{Y} \Vdash \forall X\{\forall x[\forall y(f(y, x)=1 \mapsto X y) \rightarrow X x] \rightarrow \forall x X x\}$.

ii) $\mathrm{Y} \Vdash \forall X_{1} \ldots \forall X_{k}$ $\left\{\forall x\left[\forall y\left(X_{1} y, \ldots, X_{k} y \rightarrow f(y, x) \neq 1\right), X_{1} x, \ldots, X_{k} x \rightarrow \perp\right] \rightarrow \forall x\left(X_{1} x, \ldots, X_{k} x \rightarrow \perp\right)\right\}$.

Proof. The property $\mathrm{Y} \star \xi \cdot \pi \succ \xi \star \mathrm{Y} \xi \bullet \pi$ is immediate, from theorem 1.2.

i) We take $\mathcal{X}: P \rightarrow \mathcal{P}(\boldsymbol{\Pi}), p \in P$ and $\xi \Vdash \forall x[\forall y(f(y, x)=1 \mapsto \mathcal{X} y) \rightarrow \mathcal{X} x]$. We show, by induction on the well founded relation $f(x, y)=1$, that $\mathrm{Y} \star \xi \bullet \pi \in \Perp$ for every $\pi \in \mathcal{X} p$.

Let $\pi \in \mathcal{X} p$; from (i), we get $\mathrm{Y} \star \xi \cdot \pi \succ \xi \star \mathrm{Y} \xi \bullet \pi$ and thus, it is sufficient to prove that $\xi \star Y \xi \bullet \pi \in \Perp$. By hypothesis, we have $\xi \Vdash \forall y(f(y, p)=1 \mapsto \mathcal{X} y) \rightarrow \mathcal{X} p$; thus, it suffices to show that $Y \xi \Vdash f(q, p)=1 \mapsto \mathcal{X} q$ for every $q \in P$. This is clear if $f(q, p) \neq 1$, by definition of $\mapsto$.

If $f(q, p)=1$, we must show $\mathrm{Y} \xi \Vdash \mathcal{X} q$, i.e. $\mathrm{Y} \star \xi \bullet \rho \in \Perp$ for every $\rho \in \mathcal{X} q$. But this follows from the induction hypothesis.

ii) The proof is almost the same: take $\mathcal{X}_{1}, \ldots, \mathcal{X}_{k}: P \rightarrow \mathcal{P}(\boldsymbol{\Pi}), p \in P$ and $\xi \Vdash \forall x\left[\forall y\left(\mathcal{X}_{1} y, \ldots, \mathcal{X}_{k} y \rightarrow f(y, x) \neq 1\right), \mathcal{X}_{1} x, \ldots, \mathcal{X}_{k} x \rightarrow \perp\right]$. We show, by induction on the well founded relation $f(x, y)=1$, that $\mathrm{Y} \star \xi \bullet \pi \in \Perp \Perp$ for every $\pi \in\left\|\mathcal{X}_{1} p, \ldots, \mathcal{X}_{k} p \rightarrow \perp\right\|$.

As before, we have to show that: $\mathrm{Y} \xi \Vdash \mathcal{X}_{1} q, \ldots, \mathcal{X}_{k} q \rightarrow f(q, p) \neq 1$ for all $q \in P$;

this is obvious if $f(q, p) \neq 1$. If $f(q, p)=1$, we must show $\mathrm{Y} \xi \Vdash \mathcal{X}_{1} q, \ldots, \mathcal{X}_{k} q \rightarrow \perp$, or else:

$\mathrm{Y} \star \xi \cdot \rho \in \Perp$ for every $\rho \in\left\|\mathcal{X}_{1} q, \ldots, \mathcal{X}_{k} q \rightarrow \perp\right\|$. But this follows from the induction hypothesis.

Integers, storage and recursive functions. Recall that we have a constant symbol 0 and a unary function symbol $s$ which is interpreted, in the model $\mathcal{M}$ by a bijective function $s: P \rightarrow(P \backslash\{0\})$.

And also, that we have identified $s^{n} 0$ with the integer $n$; thus, we suppose $\mathbb{N} \subset P$.

We denote by $\operatorname{int}(x)$ the formula $\forall X(\forall y(X y \rightarrow X s y), X 0 \rightarrow X x)$.

Let $u=\left(u_{n}\right)_{n \in \mathbb{N}}$ be a sequence of elements of $\boldsymbol{\Lambda}$. We define the unary predicate symbol $e_{u}$ by putting: $\left|e_{u}\left(s^{n} 0\right)\right|=\left\{u_{n}\right\} ;\left|e_{u}(p)\right|=\emptyset$ if $p \notin \mathbb{N}$.

Theorem 1.12. Let $T_{u}, S_{u} \in \boldsymbol{\Lambda}$ be such that $S_{u} \Vdash(\top \rightarrow \perp), \top \rightarrow \perp$ and:

$T_{u} \star \phi \cdot \nu \cdot \pi \succ \nu \star S_{u} \cdot \phi \bullet u_{0} \cdot \pi ; S_{u} \star \psi \cdot u_{n} \cdot \pi \succ \psi \star u_{n+1} \cdot \pi$

for every $\nu, \phi, \psi \in \boldsymbol{\Lambda}$ and $\pi \in \boldsymbol{\Pi}$. Then:

$T_{u} \Vdash \forall X \forall x\left[\left(e_{u}(x) \rightarrow X\right)\right.$, int $\left.(x) \rightarrow X\right]$.

$T_{u}$ is called a storage operator.

Proof. Let $p \in P, \phi \Vdash e_{u}(p) \rightarrow X, \nu \Vdash \operatorname{int}(p)$ and $\pi \in\|X\|$. We must show $T_{u} \star \phi \cdot \nu \cdot \pi \in$

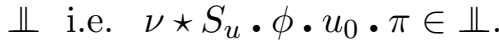

- If $p \notin \mathbb{N}$, we define the unary predicate $Y$ by putting:

$Y(q) \equiv \top$ if $q \in \mathbb{N} ; Y(q) \equiv \top \rightarrow \perp$ if $q \notin \mathbb{N}$.

Thus, we have obviously $\phi \Vdash Y(0)$ and $u_{0} \cdot \pi \in\|Y(p)\|$.

But, by hypothesis on $\nu$, we have $\nu \Vdash \forall y(Y y \rightarrow Y$ sy $), Y 0 \rightarrow Y p$.

Thus, it is sufficient to show that:

$S_{u} \Vdash \forall y(Y y \rightarrow Y s y)$, i.e. $S_{u} \Vdash Y(q) \rightarrow Y(s q)$ for every $q \in P$. 
This is clear if $q \in \mathbb{N}$, since we have $\|Y(s q)\|=\emptyset$.

If $q \notin \mathbb{N}$, we must show $S_{u} \Vdash(\top \rightarrow \perp), \top \rightarrow \perp$, which follows from the hypothesis.

- If $p \in \mathbb{N}$, we have $p=s^{p} 0$; we define the unary predicate $Y$ by putting:

$\left\|Y s^{i} 0\right\|=\left\{u_{p-i} \cdot \pi\right\}$ for $0 \leq i \leq p$ and $\|Y q\|=\emptyset$ if $q \notin\left\{s^{i} 0 ; 0 \leq i \leq p\right\}$.

By hypothesis on $\nu, \phi, \pi$, we have:

$\nu \Vdash \forall y(Y y \rightarrow Y s y), Y 0 \rightarrow Y s^{p} 0 ; \phi \Vdash Y 0 ; u_{0} \bullet \pi \in\left\|Y s^{p} 0\right\|$.

Thus, it suffices to show that $S_{u} \Vdash \forall y(Y y \rightarrow Y s y)$, i.e. $S_{u} \Vdash Y q \rightarrow Y s q$ for every $q \in P$.

This is clear if $q \notin\left\{s^{i} 0 ; 0 \leq i<p\right\}$, since then $\|Y s q\|=\emptyset$.

If $q=s^{i} 0$ with $i<p$, let $\xi \Vdash Y q$; we must show $S_{u} \star \xi \cdot u_{p-i-1} \bullet \pi \in \Perp$.

But we have $S_{u} \star \xi \cdot u_{p-i-1} \bullet \pi \succ \xi \star u_{p-i} \bullet \pi$ which is in $\Perp$, by hypothesis on $\xi$.

Notation. We define the closed c-terms $\underline{0}=\lambda x \lambda y y ; \quad \sigma=\lambda n \lambda f \lambda x(f)(n) f x$; and, for each $n \in \mathbb{N}$, we put $\underline{n}=(\sigma)^{n} \underline{0}$. We define the unary predicate symbol ent(x) by putting: $|\operatorname{ent}(n)|=\{\underline{n}\}$ if $n \in \mathbb{N}$; $|\operatorname{ent}(p)|=\emptyset$ if $p \notin \mathbb{N}$.

In other words, ent $(\mathrm{x})$ is the predicate $e_{u}(x)$ when the sequence $u$ is $(\underline{n})_{n \in \mathbb{N}}$.

\section{Theorem 1.13.}

We put $T=\lambda f \lambda n(n) S f \underline{0}$, with $S=\lambda g \lambda x(g)(\sigma) x$. Then, we have:

i) $T \Vdash \forall X \forall x((\operatorname{ent}(x) \rightarrow X)$, int $(x) \rightarrow X)$.

ii) $I \Vdash \forall x((\operatorname{ent}(x) \rightarrow \operatorname{int}(x))$.

Therefore, $T$ is a storage operator (theorem 1.12).

Proof.

i) We immediately have, by theorem 1.2 ,

$T \star \phi \cdot \nu \cdot \pi \succ \nu \star S \cdot \phi \cdot \underline{0} \cdot \pi ; S \star \psi \cdot(\sigma)^{n} \underline{0} \cdot \pi \succ \psi \star(\sigma)^{n+1} \underline{0} \cdot \pi$

for every $\nu, \phi, \psi \in \boldsymbol{\Lambda}$ and $\pi \in \boldsymbol{\Pi}$.

Now, we check that $S \Vdash(\top \rightarrow \perp), \top \rightarrow \perp$ : indeed, if $\xi \Vdash \top \rightarrow \perp$, then $S \star \xi \cdot \eta \cdot \pi \succ$ $\xi \star \sigma \eta \cdot \pi \in \Perp$ for every $\eta \in \boldsymbol{\Lambda}$ and $\pi \in \boldsymbol{\Pi}$ (by theorem 1.2).

Then, the result follows immediately, from theorem 1.12 ,

ii) We must show $I \Vdash \operatorname{ent}(p) \rightarrow \operatorname{int}(p)$ for every $p \in P$. We may suppose $p \in \mathbb{N}$ (otherwise $\operatorname{ent}(p)=\emptyset$ and the result is trivial). Then, we must show:

$I \star \sigma^{p} \underline{0} \bullet \rho \in \Perp$ knowing that $\rho \in\left\|\operatorname{int}\left(s^{p} 0\right)\right\|$.

Therefore, we can find a unary predicate $X: P \rightarrow \mathcal{P}(\boldsymbol{\Pi}), \phi \Vdash \forall y(X y \rightarrow X s y), \omega \Vdash X 0$ and $\pi \in\left\|X s^{p} 0\right\|$ such that $\rho=\phi \cdot \omega \cdot \pi$. We must show $(\sigma)^{p} \underline{0} \star \phi \cdot \omega \cdot \pi \in \Perp \Perp$. In fact, we show by recurrence on $p$, that $(\sigma)^{p} \underline{0} \star \phi \cdot \omega \cdot \pi \in \Perp$ for all $\pi \in\left\|X s^{p} 0\right\|$.

If $p=0$, let $\pi \in\|X 0\|$; we must show $\underline{0} \star \phi \cdot \omega \cdot \pi \in \Perp$, i.e. $\omega \star \pi \in \Perp$, which is clear, since $\omega \Vdash X 0$.

To move up from $p$ to $p+1$, let $\pi \in\left\|X s^{p+1} 0\right\|$. We have: $\sigma^{p+1} \underline{0} \star \phi \cdot \omega \cdot \pi \equiv(\sigma)(\sigma)^{p} \underline{0} \star \phi \cdot \omega \cdot \pi \succ \sigma \star \sigma^{p} \underline{0} \cdot \phi \cdot \omega \cdot \pi \succ \phi \star\left(\sigma^{p} \underline{0}\right) \phi \omega \cdot \pi$.

But, by induction hypothesis, we have $\sigma^{p} \underline{0} \star \phi \cdot \omega \cdot \rho \in \Perp$ for every $\rho \in\left\|X s^{p} 0\right\|$. It follows that $\left(\sigma^{p} \underline{0}\right) \phi \omega \Vdash X s^{p} 0$. Since $\phi \Vdash X s^{p} 0 \rightarrow X s^{p+1} 0$, we obtain $\phi \star\left(\sigma^{p} \underline{0}\right) \phi \omega \cdot \pi \in \Perp$.

Theorem 1.13 shows that we can use the predicate ent $(x)$ instead of int $(x)$, which greatly simplifies many computations. In particular, we define the universal quantifier restricted to integers $\forall x^{\text {int }}$ by putting $\forall x^{\text {int }} F \equiv \forall x(\operatorname{int}(x) \rightarrow F)$.

Thus, we can replace it with the universal quantifier restricted to ent $(x)$ defined as follows: $\forall x^{\text {ent }} F \equiv \forall x(\operatorname{ent}(x) \rightarrow F)$. Then, we have $\left\|\forall x^{\text {ent }} F\right\|=\left\{\underline{n} \cdot \pi ; n \in \mathbb{N}, \pi \in\left\|F\left[s^{n} 0 / x\right]\right\|\right\}$. 
Therefore, the truth value of the formula $\forall x^{\text {ent }} F$ is much simpler than the one of the formula $\forall x^{\text {int }} F$.

Theorem 1.14. Let $\phi: \mathbb{N} \rightarrow \mathbb{N}$ be a recursive function. There exists a closed $\lambda$-term $\theta$ such that, if $m \in \mathbb{N}, n=\phi(m)$ and $f$ is a $\lambda$-variable, then $\theta \underline{m} f$ reduces into $f \underline{n}$ by weak head reduction.

This is a variant of the theorem of representation of recursive functions by $\lambda$-terms. It is proved in [13].

Theorem 1.15. Let $\phi: \mathbb{N}^{k} \rightarrow \mathbb{N}$ be a recursive function. We define, in $\mathcal{M}$, a function symbol $f$, by putting $f\left(s^{m_{1}} 0, \ldots, s^{m_{k}} 0\right)=s^{n} 0$ with $n=\phi\left(m_{1}, \ldots, m_{k}\right)$; we extend $f$ on $P^{k} \backslash \mathbb{N}^{k}$ in an arbitrary way. Then, there exists a proof-like term $\theta$ such that:

$\theta \Vdash \forall x_{1} \ldots \forall x_{k}\left[\operatorname{int}\left(x_{1}\right), \ldots, \operatorname{int}\left(x_{k}\right) \rightarrow \operatorname{int}\left(f\left(x_{1}, \ldots, x_{k}\right)\right)\right]$.

Proof. For simplicity, we assume $k=1$. By theorem 1.13, it suffices to find a proof-like term $\theta$ such that $\theta \Vdash \forall x[\operatorname{ent}(x),(\operatorname{ent}(f(x)) \rightarrow \perp) \rightarrow \perp]$. In other words:

$\theta \Vdash \operatorname{ent}(p),(\operatorname{ent}(f(p)) \rightarrow \perp) \rightarrow \perp$ for every $p \in P$.

We can suppose that $p=s^{m} 0$ (otherwise, $-\operatorname{ent}(p) \mid=\emptyset$ and the result is trivial).

Thus, we have $\operatorname{ent}(p)=\{\underline{m}\}$; we must show:

$\theta \star \underline{m} \cdot \xi \cdot \pi \in \Perp$ for all $\pi \in \boldsymbol{\Pi}$ and $\xi \Vdash \operatorname{ent}\left(s^{n} 0\right) \rightarrow \perp$, with $n=\phi(m)$.

Take the $\lambda$-term $\theta$ given by theorem [1.14. From this theorem, we get:

$\theta \star \underline{m} \cdot \xi \cdot \pi \succ \xi \star \underline{n} \cdot \pi$, which is in $\Perp$, by hypothesis on $\xi$.

Remark. We have now found proof-like terms which realize all the axioms of second order arithmetic, with a function symbol for each recursive function.

\section{Standard REALizability ALGEBRAS}

A realizability algebra $\mathcal{A}$ is called standard if its set of terms $\Lambda$ and its set of stacks $\Pi$ are defined as follows:

We have a countable set $\Pi_{0}$ which is the set of stack constants.

The terms and the stacks of $\mathcal{A}$ are finite sequences of elements of the set:

$$
\Pi_{0} \cup\left\{B, C, E, I, K, W, \mathrm{cc}, \varsigma, \chi, \chi^{\prime}, \mathrm{k},(,),[,], .\right\}
$$

which are obtained by the following rules:

- $B, C, E, I, K, W, \mathrm{cc}, \varsigma, \chi, \chi^{\prime}$ are terms ;

- each element of $\Pi_{0}$ is a stack ;

- if $\xi, \eta$ are terms, then $(\xi) \eta$ is a term ;

- if $\xi$ is a term and $\pi$ a stack, then $\xi \bullet \pi$ is a stack ;

- if $\pi$ is a stack, then $\mathrm{k}[\pi]$ is a term.

A term of the form $\mathrm{k}[\pi]$ is called continuation. It will also be denoted as $\mathrm{k}_{\pi}$.

The set of processes of the algebra $\mathcal{A}$ is $\Lambda \times \Pi$.

If $\xi \in \Lambda$ and $\pi \in \Pi$, the ordered pair $(\xi, \pi)$ is denoted as $\xi \star \pi$.

Therefore, every stack has the form $\pi=\xi_{1} \ldots \xi_{n} \bullet \pi_{0}$, where $\xi_{1}, \ldots, \xi_{n} \in \Lambda$ and $\pi_{0} \in \Pi_{0}$ ( $\pi_{0}$ is a stack constant). Given a term $\tau$, we put:

$$
\pi^{\tau}=\xi_{1} \cdot \ldots \cdot \xi_{n} \cdot \tau \cdot \pi_{0} .
$$

We choose a recursive bijection from $\Pi$ onto $\mathbb{N}$, which is written $\pi \mapsto \mathrm{n}_{\pi}$. 
We define a preorder relation $\succ$, on $\Lambda \star \Pi$. It is the least reflexive and transitive relation such that, for all $\xi, \eta, \zeta \in \Lambda$ and $\pi, \varpi \in \Pi$, we have:

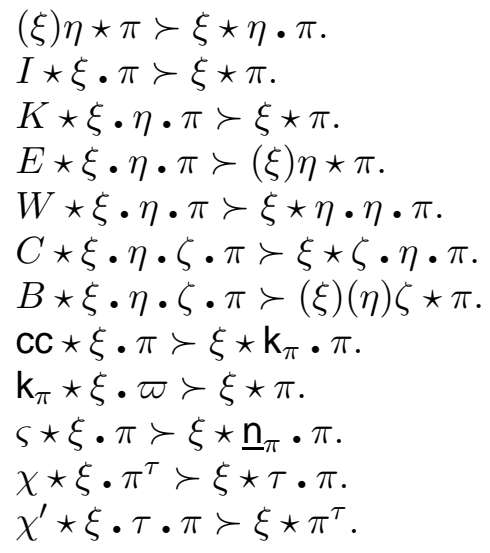

Finally, we have a subset $\Perp$ of $\Lambda \star \Pi$ which is a final segment for this preorder, which means that: $p \in \Perp, p^{\prime} \succ p \Rightarrow p^{\prime} \in \Perp \Perp$.

In other words, we ask that $\Perp$ has the following properties:

$(\xi) \eta \star \pi \notin \Perp \Rightarrow \xi \star \eta \bullet \pi \notin \Perp$.

$I \star \xi \cdot \pi \notin \Perp \Rightarrow \xi \star \pi \notin \Perp \Perp$.

$K \star \xi \cdot \eta \cdot \pi \notin \Perp \Perp H \notin \pi \notin \Perp \Perp$.

$E \star \xi \cdot \eta \cdot \pi \notin \Perp \Perp(\xi) \eta \star \pi \notin \Perp \Perp$.

$W \star \xi \cdot \eta \cdot \pi \notin \Perp \Rightarrow \xi \star \eta \cdot \eta \cdot \pi \notin \Perp \Perp$.

$C \star \xi \cdot \eta \cdot \zeta \cdot \pi \notin \Perp \# \xi \star \zeta \cdot \eta \cdot \pi \notin \Perp \Perp$.

$B \star \xi \cdot \eta \cdot \zeta \cdot \pi \notin \Perp \Rightarrow(\xi)(\eta) \zeta \star \pi \notin \Perp \Perp$.

$\mathrm{cc} \star \xi \cdot \pi \notin \Perp \Perp \xi \star \mathrm{k}_{\pi} \cdot \pi \notin \Perp \Perp$.

$\mathrm{k}_{\pi} \star \xi \cdot \varpi \notin \Perp \Rightarrow \xi \star \pi \notin \Perp$.

$\varsigma \star \xi \cdot \pi \notin \Perp \Perp \xi \star \underline{\mathrm{n}}_{\pi} \cdot \pi \notin \Perp \Perp$

$\chi \star \xi \cdot \pi^{\tau} \notin \Perp \Rightarrow \xi \star \tau \cdot \pi \notin \Perp$.

$\chi^{\prime} \star \xi \cdot \tau \cdot \pi \notin \Perp \Perp \xi \star \pi^{\tau} \notin \Perp \Perp$

Remark. Thus, the only arbitrary elements in a standard realizability algebra are the set $\Pi_{0}$ of stack constants and the set $\Perp$ of processes.

The axiom of choice for individuals (ACI). Let $\mathcal{A}$ be a standard realizability algebra and $\mathcal{M}$ a $\mathcal{A}$-model, the set of individuals of which is denoted as $P$. Then, we have:

Theorem 2.1 (ACI). For each closed formula $\forall x_{1} \ldots \forall x_{m} \forall y F$ with parameters, there exists a function $f: P^{m+1} \rightarrow P$ such that:

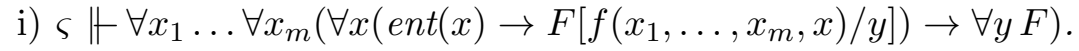

ii) $\varsigma \Vdash \forall x_{1} \ldots \forall x_{m}\left(\forall x\left(\operatorname{int}(x) \rightarrow F\left[f\left(x_{1}, \ldots, x_{m}, x\right) / y\right]\right) \rightarrow \forall y F\right)$.

Proof. For $p_{1}, \ldots, p_{m}, k \in P$, we define $f\left(p_{1}, \ldots, p_{m}, k\right)$ in an arbitrary way if $k \notin \mathbb{N}$.

If $k \in \mathbb{N}$, we have $k=\mathrm{n}_{\pi_{k}}$ for one and only one stack $\pi_{k} \in \Pi$.

We define the function $f\left(p_{1}, \ldots, p_{m}, k\right)$ by means of the axiom of choice, in such a way that, if there exists $q \in P$ such that:

$\pi_{k} \in\left\|F\left[p_{1}, \ldots, p_{m}, q\right]\right\|$, then we have $\pi_{k} \in\left\|F\left[p_{1}, \ldots, p_{m}, f\left(p_{1}, \ldots, p_{m}, k\right)\right]\right\|$.

i) We must show $\varsigma \Vdash \forall x\left(\operatorname{ent}(x) \rightarrow F\left[p_{1}, \ldots, p_{m}, f\left(p_{1}, \ldots, p_{m}, x\right)\right]\right) \rightarrow F\left[p_{1}, \ldots, p_{m}, q\right]$, for every $p_{1}, \ldots, p_{m}, q \in P$. 
Thus, let $\xi \Vdash \forall x\left(\operatorname{ent}(x) \rightarrow F\left[p_{1}, \ldots, p_{n}, f\left(p_{1}, \ldots, p_{n}, x\right)\right]\right)$ and $\pi \in\left\|F\left[p_{1}, \ldots, p_{m}, q\right]\right\|$; we must show $\varsigma \star \xi \cdot \pi \in \Perp \Perp \Perp$, that is $\xi \star \underline{\mathbf{n}}_{\pi} \cdot \pi \in \Perp \Perp \Perp$. But we have:

$\xi \Vdash \operatorname{ent}\left(\mathrm{n}_{\pi}\right) \rightarrow F\left[p_{1}, \ldots, p_{m}, f\left(p_{1}, \ldots, p_{m}, \mathrm{n}_{\pi}\right)\right]$ by hypothesis on $\xi$;

$\underline{\mathrm{n}}_{\pi} \in\left|\operatorname{ent}\left(\mathrm{n}_{\pi}\right)\right|$ by definition of ent ;

$\pi \in\left\|F\left[p_{1}, \ldots, p_{m}, f\left(p_{1}, \ldots, p_{m}, \mathrm{n}_{\pi}\right)\right]\right\|$ by hypothesis on $\pi$ and by definition of $f$.

ii) The proof is the same; in fact, (ii) is weaker than (i) since $|\operatorname{ent}(x)| \subset|\operatorname{int}(x)|$.

\section{Remarks.}

1. A seemingly simpler formulation of this axiom of choice is the existence of a function $\phi: P^{m} \rightarrow P$ such that $\forall x_{1} \ldots \forall x_{m}\left(F\left[\phi\left(x_{1}, \ldots, x_{m}\right) / y\right] \rightarrow \forall y F\right)$.

This clearly follows from theorem 2.1] simply define $\phi\left(x_{1}, \ldots, x_{m}\right)$ as $f\left(x_{1}, \ldots, x_{m}, x\right)$ for the first integer $x$ such that $\neg F\left[f\left(x_{1}, \ldots, x_{m}, x\right) / y\right]$ if there is such an integer ; otherwise, $\phi\left(x_{1}, \ldots, x_{m}\right)$ is arbitrary.

But this function $\phi$ is not a function symbol, i.e. it cannot be defined in the ground model. For this reason, we prefer to use this axiom in the form stated in theorem 2.1, which is, after all, much simpler.

2 .The axiom of dependent choice DC is a trivial consequence of ACI ; therefore theorem 2.1 shows that DC is realized by a proof-like term. Theorem 2.1 is also crucial to prove theorem 4.4 (see lemma 4.6).

3. In the following, there will be individuals which represent sets of integers (proposition 5.1), but extensionality is not realized. That is why ACI is much weaker than the usual axiom of choice. For instance, it does not imply well-ordering.

Generic models. Given a standard realizability algebra $\mathcal{A}$ and a $\mathcal{A}$-model $\mathcal{M}$, we now build a new realizability algebra $\mathcal{B}$ and a $\mathcal{B}$-model $\mathcal{N}$, which is called generic over $\mathcal{M}$. Then, we shall define the notion of forcing, which is a syntactic transformation on formulas ; it is the essential tool in order to compute truth values in the generic model $\mathcal{N}$.

Thus, we consider a standard realizability algebra $\mathcal{A}$ and a $\mathcal{A}$-model $\mathcal{M}$, the set of individuals of which is $P$.

We have a unary predicate $\mathrm{C}: P \rightarrow \mathcal{P}(\boldsymbol{\Lambda})$, a binary function $\wedge: P^{2} \rightarrow P$ and a distinguished individual $\mathbf{1} \in P$. We suppose that the data $\{\mathbf{C}, \wedge, \mathbf{1}\}$ constitute what we call a forcing structure in $\mathcal{M}$, which means that we have the following property:

There exist six proof-like terms $\alpha_{0}, \alpha_{1}, \alpha_{2}, \beta_{0}, \beta_{1}, \beta_{2}$ such that:

$\tau \in|\mathbf{C}[(p \wedge q) \wedge r]| \Rightarrow \alpha_{0} \tau \in|\mathbf{C}[p \wedge(q \wedge r)]| ;$

$\tau \in|\mathbf{C}[p]| \Rightarrow \alpha_{1} \tau \in|\mathbf{C}[p \wedge \mathbf{1}]| ;$

$\tau \in|\mathbf{C}[p \wedge q]| \Rightarrow \alpha_{2} \tau \in|\mathbf{C}[q]|$

$\tau \in|\mathbf{C}[p]| \Rightarrow \beta_{0} \tau \in|\mathbf{C}[p \wedge p]|$;

$\tau \in|\mathbf{C}[p \wedge q]| \Rightarrow \beta_{1} \tau \in|\mathbf{C}[q \wedge p]| ;$

$\tau \in|\mathrm{C}[((p \wedge q) \wedge r) \wedge s]| \Rightarrow \beta_{2} \tau \in|\mathbf{C}[(p \wedge(q \wedge r)) \wedge s]|$.

We shall call $\mathbf{C}$-expression any finite sequence of symbols of the form $\gamma=\left(\delta_{0}\right)\left(\delta_{1}\right) \ldots\left(\delta_{k}\right)$ where each $\delta_{i}$ is one of the proof-like terms $\alpha_{0}, \alpha_{1}, \alpha_{2}, \beta_{0}, \beta_{1}, \beta_{2}$.

Such an expression is not a C-term, but $\gamma \tau$ is, for every C-term $\tau$;

the term $\gamma \tau=\left(\delta_{0}\right)\left(\delta_{1}\right) \ldots\left(\delta_{k}\right) \tau$ will also be written $(\gamma) \tau$.

Notation. A $\wedge$-term is, by definition, a term which is written with the variables $p_{1}, \ldots, p_{k}$, the constant 1 and the binary function symbol $\wedge$. Let $t\left(p_{1}, \ldots, p_{k}\right), u\left(p_{1}, \ldots, p_{k}\right)$ be two $\wedge$ terms. The notation: 


$$
\gamma:: t\left(p_{1}, \ldots, p_{k}\right) \Rightarrow u\left(p_{1}, \ldots, p_{k}\right)
$$

means that $\gamma$ is a $\mathbf{C}$-expression such that $\tau \in\left|\mathbf{C}\left[t\left(p_{1}, \ldots, p_{k}\right)\right]\right| \Rightarrow(\gamma) \tau \in\left|\mathbf{C}\left[u\left(p_{1}, \ldots, p_{k}\right)\right]\right|$. Thus, with this notation, the above hypothesis can be written as follows:

$\alpha_{0}::(p \wedge q) \wedge r \Rightarrow p \wedge(q \wedge r) ; \quad \alpha_{1}:: p \Rightarrow p \wedge \mathbf{1} ; \alpha_{2}:: p \wedge q \Rightarrow q ;$

$\beta_{0}:: p \Rightarrow p \wedge p ; \beta_{1}:: p \wedge q \Rightarrow q \wedge p ; \beta_{2}::((p \wedge q) \wedge r) \wedge s \Rightarrow(p \wedge(q \wedge r)) \wedge s$.

Lemma 2.2. There exist C-expressions $\beta_{0}^{\prime}, \beta_{1}^{\prime}, \beta_{2}^{\prime}, \beta_{3}, \beta_{3}^{\prime}$ such that:

$\beta_{0}^{\prime}:: p \wedge q \Rightarrow(p \wedge q) \wedge q ; \beta_{1}^{\prime}::(p \wedge q) \wedge r \Rightarrow(q \wedge p) \wedge r ; \quad \beta_{2}^{\prime}:: p \wedge(q \wedge r) \Rightarrow(p \wedge q) \wedge r ;$

$\beta_{3}:: p \wedge(q \wedge r) \Rightarrow p \wedge(r \wedge q) ; \beta_{3}^{\prime}::(p \wedge(q \wedge r)) \wedge s \Rightarrow(p \wedge(r \wedge q)) \wedge s$.

Proof. We write the sequence of transformations, with the C-expressions which perform them:

- $\beta_{0}^{\prime}=\left(\beta_{1}\right)\left(\alpha_{2}\right)\left(\alpha_{0}\right)\left(\beta_{0}\right)$.

$p \wedge q ; \beta_{0} ;(p \wedge q) \wedge(p \wedge q) ; \alpha_{0} ; p \wedge(q \wedge(p \wedge q)) ; \alpha_{2} ; q \wedge(p \wedge q) ; \beta_{1} ;(p \wedge q) \wedge q$.

- $\beta_{2}^{\prime}=\left(\beta_{1}\right)\left(\alpha_{0}\right)\left(\beta_{1}\right)\left(\alpha_{0}\right)\left(\beta_{1}\right)$.

$p \wedge(q \wedge r) ; \beta_{1} ;(q \wedge r) \wedge p ; \alpha_{0} ; q \wedge(r \wedge p) ; \beta_{1} ;(r \wedge p) \wedge q ; \alpha_{0} ; r \wedge(p \wedge q) ; \beta_{1} ;(p \wedge q) \wedge r$.

- $\beta_{1}^{\prime}=\left(\alpha_{2}\right)\left(\alpha_{0}\right)\left(\beta_{2}\right)\left(\beta_{1}\right)\left(\alpha_{0}\right)\left(\alpha_{2}\right)\left(\beta_{1}\right)\left(\beta_{2}^{\prime}\right)\left(\beta_{0}^{\prime}\right)\left(\beta_{1}\right)$.

$(p \wedge q) \wedge r ; \beta_{1} ; r \wedge(p \wedge q) ; \beta_{0}^{\prime}(r \wedge(p \wedge q)) \wedge(p \wedge q) ; \beta_{2}^{\prime} ;((r \wedge(p \wedge q)) \wedge p) \wedge q ; \beta_{1} ; q \wedge((r \wedge(p \wedge q)) \wedge p)$;

$\alpha_{2} ;(r \wedge(p \wedge q)) \wedge p ; \alpha_{0} ; r \wedge((p \wedge q) \wedge p) ; \beta_{1} ;((p \wedge q) \wedge p) \wedge r ; \beta_{2} ;(p \wedge(q \wedge p)) \wedge r ; \alpha_{0} ;$

$p \wedge((q \wedge p) \wedge r) ; \alpha_{2} ;(q \wedge p) \wedge r$.

- $\beta_{3}=\left(\beta_{1}\right)\left(\beta_{1}^{\prime}\right)\left(\beta_{1}\right)$.

$p \wedge(q \wedge r) ; \beta_{1} ;(q \wedge r) \wedge p ; \beta_{1}^{\prime} ;(r \wedge q) \wedge p ; \beta_{1} ; p \wedge(r \wedge q)$.

- $\beta_{3}^{\prime}=\left(\beta_{1}^{\prime}\right)\left(\beta_{2}^{\prime}\right)\left(\beta_{1}^{\prime}\right)\left(\alpha_{0}\right)\left(\beta_{1}^{\prime}\right)$.

$(p \wedge(q \wedge r)) \wedge s ; \beta_{1}^{\prime} ;((q \wedge r) \wedge p) \wedge s ; \alpha_{0} ;(q \wedge r) \wedge(p \wedge s) ; \beta_{1}^{\prime} ;(r \wedge q) \wedge(p \wedge s) ; \beta_{2}^{\prime} ;((r \wedge q) \wedge p) \wedge s ;$

$\beta_{1}^{\prime} ;(p \wedge(r \wedge q)) \wedge s$.

Lemma 2.3. Let $t$ be $a \wedge$-term and $p$ a variable of $t$. Then, there exists a C-expression $\gamma$ such that $\gamma:: t \Rightarrow t \wedge p$.

Proof. By induction on the number of symbols of $t$ which stand after the last occurrence of $p$. If this number is 0 , then $t=p$ or $t=u \wedge p$. Then, we have $\gamma=\beta_{0}$ or $\beta_{0}^{\prime}$ (lemma 2.2).

Otherwise, we have $t=u \wedge v$; if the last occurrence of $p$ is in $u$, the recurrence hypothesis gives $\gamma^{\prime}:: v \wedge u \Rightarrow(v \wedge u) \wedge p$. Then, we have $\gamma=\left(\beta_{1}^{\prime}\right)\left(\gamma^{\prime}\right)\left(\beta_{1}\right)$.

If the last occurrence of $p$ is in $v$, we have $v=v_{0} \wedge v_{1}$. If this occurrence is in $v_{0}$, the recurrence hypothesis gives $\gamma^{\prime}:: u \wedge\left(v_{1} \wedge v_{0}\right) \Rightarrow\left(u \wedge\left(v_{1} \wedge v_{0}\right)\right) \wedge p$. We put $\gamma=\left(\beta_{3}^{\prime}\right)\left(\gamma^{\prime}\right)\left(\beta_{3}\right)$ (lemma 2.2).

If this occurrence is in $v_{1}$, the recurrence hypothesis gives $\gamma^{\prime}::\left(u \wedge v_{0}\right) \wedge v_{1} \Rightarrow\left(\left(u \wedge v_{0}\right) \wedge v_{1}\right) \wedge p$. Then, we put $\gamma=\left(\beta_{2}\right)\left(\gamma^{\prime}\right)\left(\beta_{2}^{\prime}\right)$.

Lemma 2.4. Let $t, u$ be two $\wedge$-terms such that each variable of $u$ appears in $t$. Then, there exists a $\mathrm{C}$-expression $\gamma$ such that $\gamma:: t \Rightarrow t \wedge u$.

Proof by recurrence on the length of $u$.

If $u=\mathbf{1}$, then $\gamma=\alpha_{1}$; if $u$ is a variable, we apply lemma 2.3.

If $u=v \wedge w$, the recurrence hypothesis gives $\gamma^{\prime}:: t \Rightarrow t \wedge v$ and also $\gamma^{\prime \prime}:: t \wedge v \Rightarrow(t \wedge v) \wedge w$. Then, we put $\gamma=\left(\alpha_{0}\right)\left(\gamma^{\prime \prime}\right)\left(\gamma^{\prime}\right)$.

Theorem 2.5. Let $t, u$ be two $\wedge$-terms such that each variable of $u$ appears in $t$. Then, there exists a C-expression $\gamma$ such that $\gamma:: t \Rightarrow u$.

Proof. By lemma 2.4, we have $\gamma^{\prime}:: t \Rightarrow t \wedge u$. Thus, we can put $\gamma=\left(\alpha_{2}\right)\left(\gamma^{\prime}\right)$. 
Corollary 2.6. There exist C-expressions $\gamma_{I}, \gamma_{K}, \gamma_{E}, \gamma_{W}, \gamma_{C}, \gamma_{B}, \gamma_{\mathrm{cc}}, \gamma_{\mathrm{k}}$ such that:

$\gamma_{I}:: p \wedge q \Rightarrow q ; \gamma_{K}:: \mathbf{1} \wedge(p \wedge(q \wedge r)) \Rightarrow p \wedge r ; \gamma_{E}:: \mathbf{1} \wedge(p \wedge(q \wedge r)) \Rightarrow(p \wedge q) \wedge r ;$

$\gamma_{W}:: \mathbf{1} \wedge(p \wedge(q \wedge r)) \Rightarrow p \wedge(q \wedge(q \wedge r)) ; \gamma_{C}:: \mathbf{1} \wedge(p \wedge(q \wedge(r \wedge s))) \Rightarrow p \wedge(r \wedge(q \wedge s)) ;$

$\gamma_{B}:: \mathbf{1} \wedge(p \wedge(q \wedge(r \wedge s))) \Rightarrow(p \wedge(q \wedge r)) \wedge s ; \quad \gamma_{\mathrm{cc}}:: \mathbf{1} \wedge(p \wedge q) \Rightarrow p \wedge(q \wedge q) ;$

$\gamma_{\mathrm{k}}:: p \wedge(q \wedge r) \Rightarrow q \wedge p$.

The algebra $\mathcal{B}$. We define now a new realizability algebra $\mathcal{B}=(\boldsymbol{\Lambda}, \boldsymbol{\Pi}, \boldsymbol{\Lambda} \star \boldsymbol{\Pi}, \mathbb{\Perp})$ : its set of terms is $\boldsymbol{\Lambda}=\Lambda \times P$, its set of stacks is $\Pi=\Pi \times P$ and its set of processes is $\Lambda \star \Pi=(\Lambda \star \Pi) \times P$.

The distinguished subset $\Perp_{\mathcal{B}}$ of $\boldsymbol{\Lambda} \star \Pi$ is denoted by $\Perp$. It is defined as follows:

$(\xi \star \pi, p) \in \mathbb{\Perp} \Leftrightarrow(\forall \tau \in \mathrm{C}[p]) \xi \star \pi^{\tau} \in \Perp \Perp$

For $(\xi, p) \in \boldsymbol{\Lambda}$ and $(\pi, q) \in \boldsymbol{\Pi}$, we put:

$(\xi, p) \star(\pi, q)=(\xi \star \pi, p \wedge q) ;$

$(\xi, p) \cdot(\pi, q)=(\xi \cdot \pi, p \wedge q)$

For $(\xi, p),(\eta, q) \in \boldsymbol{\Lambda}$, we put:

$(\xi, p)(\eta, q)=\left(\bar{\alpha}_{0} \xi \eta, p \wedge q\right)$ with $\bar{\alpha}_{0}=\lambda x(\chi) \lambda y\left(\chi^{\prime} x\right)\left(\alpha_{0}\right) y$.

Lemma 2.7. For each C-expression $\gamma$, we put $\bar{\gamma}=\lambda x(\chi) \lambda y\left(\chi^{\prime} x\right)(\gamma) y$.

Then, we have $\bar{\gamma} \star \xi \cdot \pi^{\tau} \succ \xi \star \pi^{\gamma \tau}$.

Proof. This is immediate, by means of theorem 1.2 .

We could take also $\bar{\gamma}=(\chi) \lambda x \lambda y\left(\chi^{\prime} y\right)(\gamma) x$.

Proposition 2.8. If we have $\gamma:: t\left(p_{1}, \ldots, p_{k}\right) \Rightarrow u\left(p_{1}, \ldots, p_{k}\right)$, then:

$\left(\bar{\gamma} \star \xi \cdot \pi, t\left(p_{1}, \ldots, p_{k}\right)\right) \succ\left(\xi \star \pi, u\left(p_{1}, \ldots, p_{k}\right)\right)$.

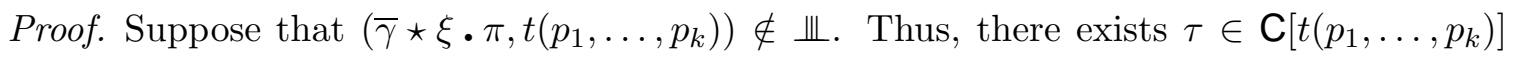
such that:

$\bar{\gamma} \star \xi \cdot \pi^{\tau} \notin \Perp$. Therefore, we have $\xi \star \pi^{\gamma \tau} \notin \Perp$ et $\gamma \tau \in \mathrm{C}\left[u\left(p_{1}, \ldots, p_{k}\right)\right]$. It follows that: $\left(\xi \star \pi, u\left(p_{1}, \ldots, p_{k}\right)\right) \notin \mathbb{\Perp}$.

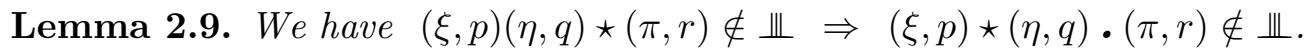

Proof. By hypothesis, we have $\left(\bar{\alpha}_{0} \xi \eta \star \pi,(p \wedge q) \wedge r\right) \notin \mathbb{\Perp}$; thus, there exists $\tau \in \mathrm{C}[(p \wedge q) \wedge r]$ such that $\bar{\alpha}_{0} \xi \eta \star \pi^{\tau} \notin \Perp$. By lemma 2.7, we have $\xi \star \eta \bullet \pi^{\alpha_{0} \tau} \notin \Perp ;$ since $\alpha_{0} \tau \in \mathrm{C}[p \wedge(q \wedge r)]$, we have $(\xi \star \eta \cdot \pi, p \wedge(q \wedge r)) \notin \Perp$ and thus $(\xi, p) \star(\eta, q) \cdot(\pi, r) \notin \mathbb{\Perp}$.

We define the elementary combinators $\mathbf{B}, \mathbf{C}, \mathbf{E}, \mathbf{I}, \mathbf{K}, \mathbf{W}, \mathbf{c c}$ of the algebra $\mathcal{B}$ by putting: $\mathbf{B}=\left(B^{*}, \mathbf{1}\right) ; \mathbf{C}=\left(C^{*}, \mathbf{1}\right) ; \mathbf{E}=\left(E^{*}, \mathbf{1}\right) ; \mathbf{I}=\left(I^{*}, \mathbf{1}\right) ; \mathbf{K}=\left(K^{*}, \mathbf{1}\right) ; \mathbf{W}=\left(W^{*}, \mathbf{1}\right) ;$ $\mathbf{c c}=\left(\mathrm{CC}^{*}, \mathbf{1}\right)$ with $B^{*}=\lambda x \lambda y \lambda z\left(\bar{\gamma}_{B}\right)\left(\bar{\alpha}_{0} x\right)\left(\bar{\alpha}_{0}\right) y z ; C^{*}=\bar{\gamma}_{C} C ; E^{*}=\lambda x \lambda y\left(\bar{\gamma}_{E}\right)\left(\bar{\alpha}_{0}\right) x y ; I^{*}=\bar{\gamma}_{I} I$; $K^{*}=\bar{\gamma}_{K} K ; W^{*}=\bar{\gamma}_{W} W ; \mathbf{c c}^{*}=(\chi) \lambda x \lambda y(\mathbf{c c}) \lambda k\left(\left(\chi^{\prime} y\right)\left(\gamma_{\mathrm{cc}}\right) x\right)(\chi) \lambda x \lambda y(k)\left(\chi^{\prime} y\right)\left(\gamma_{\mathrm{k}}\right) x$.

We put $\mathbf{k}_{(\pi, p)}=\left(\mathbf{k}_{\pi}^{*}, p\right)$ with $\mathbf{k}_{\pi}^{*}=(\chi) \lambda x \lambda y\left(\mathbf{k}_{\pi}\right)\left(\chi^{\prime} y\right)\left(\gamma_{\mathbf{k}}\right) x$.

Theorem 2.10. For every $\tilde{\xi}, \tilde{\eta}, \tilde{\zeta} \in \boldsymbol{\Lambda}$ and $\tilde{\pi}, \tilde{\varpi} \in \boldsymbol{\Pi}$, we have:

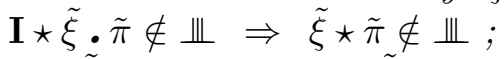

$\mathbf{K} \star \tilde{\xi} \cdot \tilde{\eta} \cdot \tilde{\pi} \notin \mathbb{\Perp} \Rightarrow \tilde{\xi} \star \tilde{\pi} \notin \mathbb{\Perp} ;$

$\mathbf{E} \star \tilde{\xi} \cdot \tilde{\eta} \cdot \tilde{\pi} \notin \mathbb{\Perp} \Rightarrow(\tilde{\xi}) \tilde{\eta} \star \tilde{\pi} \notin \mathbb{\Perp} ;$

$\mathbf{W} \star \tilde{\xi} \cdot \tilde{\eta} \cdot \tilde{\pi} \notin \mathbb{\Perp} \Rightarrow \tilde{\xi} \star \tilde{\eta} \cdot \tilde{\eta} \cdot \tilde{\pi} \notin \mathbb{\Perp}$. 


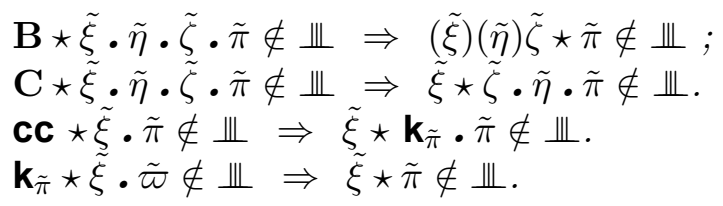

Proof. We shall prove only the cases $\mathbf{W}, \mathbf{B}, \mathbf{k}_{\tilde{\pi}}, \mathbf{c c}$.

We put $\tilde{\xi}=(\xi, p), \tilde{\eta}=(\eta, q), \tilde{\zeta}=(\zeta, r), \tilde{\pi}=(\pi, s), \tilde{\varpi}=(\varpi, q)$.

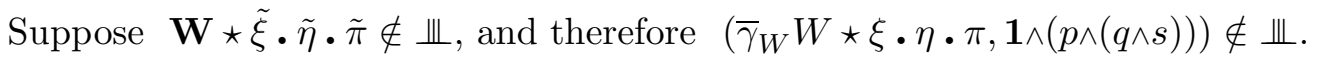

Thus, there exists $\tau \in \mathrm{C}[\mathbf{1} \wedge(p \wedge(q \wedge s))]$ such that $\bar{\gamma}_{W} W \star \xi \cdot \eta \cdot \pi^{\tau} \notin \Perp \Perp$.

Since $\bar{\gamma}_{W} W \star \xi \cdot \eta \cdot \pi^{\tau} \succ \xi \star \eta \cdot \eta \cdot \pi^{\gamma_{W} \tau}$, we have $\xi \star \eta \cdot \eta \cdot \pi^{\gamma_{W} \tau} \notin \Perp$.

But $\gamma_{W} \tau \in \mathrm{C}[p \wedge(q \wedge(q \wedge s))]$ and it follows that $\tilde{\xi} \star \tilde{\eta} \cdot \tilde{\eta} \cdot \tilde{\pi} \notin \mathbb{\Perp}$.

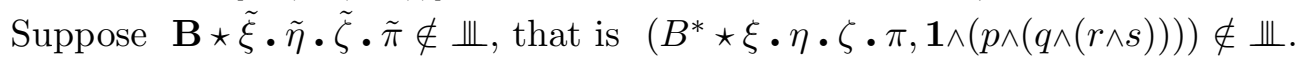

Thus, there exists $\tau \in \mathrm{C}[\mathbf{1} \wedge(p \wedge(q \wedge(r \wedge s)))]$ such that $B^{*} \star \xi \cdot \eta \cdot \zeta \cdot \pi^{\tau} \notin \Perp$.

But, we have $B^{*} \star \xi \cdot \eta \cdot \zeta \cdot \pi^{\tau} \succ\left(\bar{\gamma}_{B}\right)\left(\bar{\alpha}_{0} \xi\right)\left(\bar{\alpha}_{0}\right) \eta \zeta \star \pi^{\tau}$ (by theorem 1.2)

$\succ\left(\bar{\alpha}_{0} \xi\right)\left(\bar{\alpha}_{0}\right) \eta \zeta \star \pi^{\gamma_{B} \tau}$ (by lemma 2.7). Therefore, we have $\left(\bar{\alpha}_{0} \xi\right)\left(\bar{\alpha}_{0}\right) \eta \zeta \star \pi^{\gamma_{B} \tau} \notin \Perp$.

But $\gamma_{B} \tau \in \mathrm{C}[(p \wedge(q \wedge r)) \wedge s]$ and thus, we have:

$\left(\left(\bar{\alpha}_{0} \xi\right)\left(\bar{\alpha}_{0}\right) \eta \zeta \star \pi,(p \wedge(q \wedge r)) \wedge s\right) \notin \Perp \mathbb{H}$, in other words $(\tilde{\xi})(\tilde{\eta}) \tilde{\zeta} \star \tilde{\pi} \notin \Perp \Perp$.

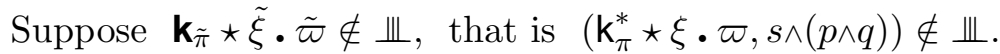

Thus, there exists $\tau \in \mathrm{C}[s \wedge(p \wedge q)]$ such that $\mathrm{k}_{\pi}^{*} \star \xi \cdot \varpi^{\tau} \notin \Perp$. But we have:

$\mathrm{k}_{\pi}^{*} \star \xi \cdot \varpi^{\tau} \succ \lambda x \lambda y\left(\mathrm{k}_{\pi}\right)\left(\chi^{\prime} y\right)\left(\gamma_{\mathrm{k}}\right) x \star \tau \cdot \xi \cdot \varpi \succ\left(\mathbf{k}_{\pi}\right)\left(\chi^{\prime} \xi\right)\left(\gamma_{\mathrm{k}}\right) \tau \star \varpi$ (by theorem 1.2) $\succ\left(\chi^{\prime} \xi\right)\left(\gamma_{\mathrm{k}}\right) \tau \star \pi \succ \chi^{\prime} \star \xi \cdot \gamma_{\mathrm{k}} \tau \cdot \pi \succ \xi \star \pi^{\gamma_{\mathrm{k}} \tau}$.

Thus, we have $\xi \star \pi^{\gamma_{\mathrm{k}} \tau} \notin \Perp$; but, since $\gamma_{\mathrm{k}} \tau \in \mathrm{C}[p \wedge s]$, we get $\tilde{\xi} \star \tilde{\pi} \notin \mathbb{\Perp}$.

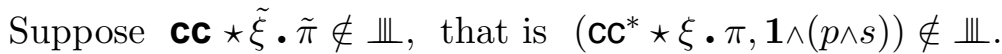

Thus, there exists $\tau \in \mathrm{C}[\mathbf{1} \wedge(p \wedge s)]$ such that $\mathrm{Cc}^{*} \star \xi \cdot \pi^{\tau} \notin \Perp \Perp$. But we have:

$\mathrm{cc}^{*} \star \xi \cdot \pi^{\tau} \succ \lambda x \lambda y(\mathrm{cc}) \lambda k\left(\left(\chi^{\prime} y\right)\left(\gamma_{\mathrm{cc}}\right) x\right)(\chi) \lambda x \lambda y(k)\left(\chi^{\prime} y\right)\left(\gamma_{\mathrm{k}}\right) x \star \tau \cdot \xi \cdot \pi$

$\succ(\mathrm{cc}) \lambda k\left(\left(\chi^{\prime} \xi\right)\left(\gamma_{\mathrm{cc}}\right) \tau\right)(\chi) \lambda x \lambda y(k)\left(\chi^{\prime} y\right)\left(\gamma_{\mathrm{k}}\right) x \star \pi$

$\succ\left(\left(\chi^{\prime} \xi\right)\left(\gamma_{\mathrm{cc}}\right) \tau\right)(\chi) \lambda x \lambda y\left(\mathrm{k}_{\pi}\right)\left(\chi^{\prime} y\right)\left(\gamma_{\mathrm{k}}\right) x \star \pi \succ \chi^{\prime} \star \xi \cdot \gamma_{\mathrm{cc}} \tau \cdot(\chi) \lambda x \lambda y\left(\mathrm{k}_{\pi}\right)\left(\chi^{\prime} y\right)\left(\gamma_{\mathrm{k}}\right) x \cdot \pi$

$\succ \xi \star(\chi) \lambda x \lambda y\left(\mathrm{k}_{\pi}\right)\left(\chi^{\prime} y\right)\left(\gamma_{\mathrm{k}}\right) x \cdot \pi^{\gamma_{\mathrm{cc}} \tau} \equiv \xi \star \mathrm{k}_{\pi}^{*} \cdot \pi^{\gamma_{\mathrm{cc}} \tau}$.

It follows that $\xi \star \mathrm{k}_{\pi}^{*} \cdot \pi^{\gamma_{\mathrm{cc}} \tau} \notin \Perp \Perp$. But we have $\gamma_{\mathrm{cc}} \tau \in \mathrm{C}[p \wedge(s \wedge s)]$ and it follows that we

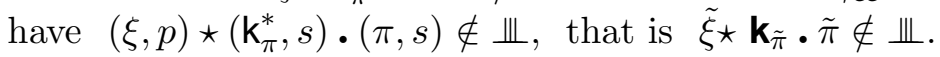

We have now completely defined the realizability algebra $\mathcal{B}$.

For each closed $\mathrm{c}$-term $t$ (proof-like term), let us denote by $t_{\mathcal{B}}$ its value in the algebra $\mathcal{B}$ (its value in the standard algebra $\mathcal{A}$ is $t$ itself). Thus, we have $t_{\mathcal{B}}=\left(t^{*}, \mathbf{1}_{t}\right)$, where $t^{*}$ is a proof-like term and $\mathbf{1}_{t}$ a condition written with $\mathbf{1}, \wedge$ and parentheses, which are obtained as follows, by recurrence on $t$ :

- If $t$ is an elementary combinator $B, C, E, I, K, W, \mathrm{cc}$, then $t^{*}$ is already defined $; \mathbf{1}_{t}=\mathbf{1}$.

- $(t u)^{*}=\bar{\alpha}_{0} t^{*} u^{*} ; \mathbf{1}_{t u}=\mathbf{1}_{t} \wedge \mathbf{1}_{u}$.

The model $\mathcal{N}$. The $\mathcal{B}$-model $\mathcal{N}$ has the same set $P$ of individuals and the same functions as $\mathcal{M}$.

By definition, the $k$-ary predicates of $\mathcal{N}$ are the applications from $P^{k}$ into $\mathcal{P}(\boldsymbol{\Pi})$. But, since $\Pi=\Pi \times P$, they are the same as the applications from $P^{k+1}$ into $\mathcal{P}(\Pi)$, i.e. the $k+1$-ary predicates of the model $\mathcal{M}$.

Each predicate constant $\mathrm{R}$, of arity $k$, is interpreted, in the model $\mathcal{M}$, by an application 
$\mathrm{R}_{\mathcal{M}}$ from $P^{k}$ into $\mathcal{P}(\Lambda)$. In the model $\mathcal{N}$, this predicate constant is interpreted by the application $\mathrm{R}_{\mathcal{N}}: P^{k} \rightarrow \mathcal{P}(\boldsymbol{\Lambda})$, where $\mathrm{R}_{\mathcal{N}}\left(p_{1}, \ldots, p_{k}\right)=\mathrm{R}_{\mathcal{M}}\left(p_{1}, \ldots, p_{k}\right) \times\{\mathbf{1}\}$.

For each closed formula $F$, with parameters in $\mathcal{N}$, its truth value, which is a subset of $\Pi$, will be denoted by $\||F|\|$. We shall write $(\xi, p) \| \models F$ to mean that $(\xi, p) \in \boldsymbol{\Lambda}$ realizes $F$, in other words $(\forall \pi \in \Pi)(\forall q \in P)(((\pi, q) \in\||\|F \mid\|) \Rightarrow(\xi, p) \star(\pi, q) \in \mathbb{H})$.

\section{Theorem 2.11.}

If we have $\vdash t$ : $A$ in classical second order logic, where $A$ is a closed formula, then $t_{\mathcal{B}}=\left(t^{*}, \mathbf{1}_{t}\right) \| \leftarrow A$.

Proof. Immediate application of theorem 1.3 (adequacy lemma) in the $\mathcal{B}$-model $\mathcal{N}$.

\section{Proposition 2.12.}

i) If $(\xi, \mathbf{1}) \|-F$, then $(\bar{\gamma} \xi, p) \| \mapsto F$ for each $p \in P$, with $\gamma:: p \wedge q \Rightarrow \mathbf{1} \wedge q$.

ii) Let $\xi, \eta \in \Lambda$ be such that $\xi \star \pi \succ \eta \star \pi$ for each $\pi \in \Pi$. Then, we have:

$(\xi \star \pi, p) \notin \mathbb{\Perp} \Rightarrow(\eta \star \pi, p) \notin \mathbb{\Perp}$ for every $\pi \in \Pi$ and $p \in P$;

$(\eta, p)\|\models F \Rightarrow(\xi, p)\|-F$ for every closed formula $F$.

Proof.

i) We must show that, for each $(\pi, q) \in\||\|F \mid\|$, we have $(\bar{\gamma} \xi, p) \star(\pi, q) \in \Perp \Perp \Perp$, that is:

$(\bar{\gamma} \xi \star \pi, p \wedge q) \in \mathbb{\Perp}$. Thus, let $\tau \in \mathrm{C}[p \wedge q]$, so that $\gamma \tau \in \mathrm{C}[\mathbf{1} \wedge q]$.

Since we have, by hypothesis, $(\xi \star \pi, \mathbf{1} \wedge q) \in \mathbb{\Perp}$, it follows that $\xi \star \pi^{\gamma \tau} \in \Perp \Perp$ and therefore $\bar{\gamma} \xi \star \pi^{\tau} \in \Perp$.

ii) By hypothesis, there exists $\tau \in \mathrm{C}[p]$ such that $\xi \star \pi^{\tau} \notin \Perp$. Thus, we have $\eta \star \pi^{\tau} \notin \Perp$, so that $(\eta \star \pi, p) \notin \mathbb{\Perp}$.

Let $(\pi, q) \in\|\mid\| F \|$; we have $(\eta, p) \star(\pi, q) \in \mathbb{\Perp}$, that is $(\eta \star \pi, p \wedge q) \in \Perp$. From what we have

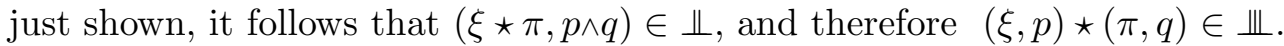

The integers of the model $\mathcal{N}$. Recall that we have put:

$\sigma=\lambda n \lambda f \lambda x(f)(n) f x, \underline{0}=\lambda x \lambda y y$ and $\underline{n}=(\sigma)^{n} \underline{0}$ for every integer $n$.

Thus, we have $\sigma_{\mathcal{B}}=\left(\sigma^{*}, \mathbf{1}_{\sigma}\right)$ and $\underline{n}_{\mathcal{B}}=\left((\sigma)^{n} \underline{0}\right)_{\mathcal{B}}=\left(\underline{n}^{*}, \mathbf{1}_{\underline{n}}\right)$.

Therefore $\underline{0}_{\mathcal{B}}=(K I)_{\mathcal{B}}=\left(K^{*}, \mathbf{1}\right)\left(I^{*}, \mathbf{1}\right)$ and $\underline{n+1_{\mathcal{B}}}=\sigma_{\mathcal{B}} \underline{\underline{n}}_{\mathcal{B}}=\left(\sigma^{*}, \mathbf{1}_{\sigma}\right)\left(\underline{n}^{*}, \mathbf{1}_{\underline{n}}\right)$.

Thus, the recursive definitions of $\underline{n}^{*}, \mathbf{1}_{\underline{n}}$ are the following:

$\left.\underline{0}^{*}=\bar{\alpha}_{0} K^{*} I^{*} ; \underline{(n+1}\right)^{*}=\bar{\alpha}_{0} \sigma^{*} \underline{n}^{*} ;$

$\mathbf{1}_{\underline{0}}=\mathbf{1} \wedge \mathbf{1} ; \mathbf{1}_{\underline{n+1}}=\mathbf{1}_{\sigma} \wedge \mathbf{1}_{\underline{n}}$.

We can define the unary predicate $\operatorname{ent}(x)$ in the model $\mathcal{N}$ in two distinct ways:

i) From the predicate $\operatorname{ent}(x)$ of the model $\mathcal{M}$, by putting:

$\left|\operatorname{ent}\left(s^{n} 0\right)\right|=\{(\underline{n}, \mathbf{1})\} ;|\operatorname{ent}(p)|=\emptyset$ if $p \notin \mathbb{N}$.

ii) By using directly the definition of $\operatorname{ent}(\mathrm{x})$ in the model $\mathcal{N}$; we denote this predicate by $\operatorname{ent}_{\mathcal{N}}(x)$. Therefore, we have:

$\left|\operatorname{ent}_{\mathcal{N}}\left(s^{n} 0\right)\right|=\left\{\underline{n}_{\mathcal{B}}\right\} ;\left|\operatorname{ent}_{\mathcal{N}}(p)\right|=\emptyset$ if $p \notin \mathbb{N}$.

From theorem 1.13, applied in the model $\mathcal{N}$, we know that the predicates $\operatorname{int}(x)$ and $\operatorname{ent}_{\mathcal{N}}(x)$ are interchangeable. Theorem 2.13 shows that the predicates int $(x)$ and ent $(x)$ are also interchangeable. Thus, we have three predicates which define the integers in the model $\mathcal{N}$; it is the predicate ent $(x)$ that we shall mostly use in the sequel. In particular, we shall often replace the quantifier $\forall x^{\text {int }}$ with $\forall x^{\text {ent }}$. 


\section{Theorem 2.13.}

There exist two proof-like terms $T, J$ such that:

i) $(T, \mathbf{1}) \| \forall \forall X \forall x((\operatorname{ent}(x) \rightarrow X)$, int $(x) \rightarrow X)$.

ii) $(J, \mathbf{1}) \| \forall \forall x(\operatorname{ent}(x) \rightarrow \operatorname{int}(x))$.

Proof.

i) We apply theorem 1.12 to the sequence $u: \mathbb{N} \rightarrow \boldsymbol{\Lambda}$ defined by $u_{n}=(\underline{n}, \mathbf{1})$.

We are looking for two proof-like terms $T, S$ such that:

$(S, \mathbf{1}) \star(\psi, p) \cdot(\underline{n}, \mathbf{1}) \cdot(\pi, r) \succ(\psi, p) \star(\underline{n+1}, \mathbf{1}) \cdot(\pi, r) ;(S, \mathbf{1}) \| \leftarrow \rightarrow \perp, \top \rightarrow \perp$.

$(T, \mathbf{1}) \star(\phi, p) \cdot(\nu, q) \cdot(\pi, r) \succ(\nu, q) \star(S, \mathbf{1}) \cdot(\phi, p) \cdot(\underline{0}, \mathbf{1}) \cdot(\pi, r)$.

Then theorem 1.12 will give the desired result:

$(T, \mathbf{1}) \| \forall \forall X \forall x((\operatorname{ent}(x) \rightarrow X), \operatorname{int}(x) \rightarrow X)$.

We put $S=\lambda f \lambda x(\bar{\gamma} f)(\sigma) x$, with $\gamma:: \mathbf{1} \wedge(p \wedge(q \wedge r)) \Rightarrow p \wedge(q \wedge r)$.

Then, we have $(S, \mathbf{1}) \star(\psi, p) \cdot(\nu, q) \cdot(\pi, r) \equiv(S \star \psi \cdot \nu \cdot \pi, \mathbf{1} \wedge(p \wedge(q \wedge r))) \succ$

$(\bar{\gamma} \psi \star \sigma \nu \cdot \pi, \mathbf{1} \wedge(p \wedge(q \wedge r)))$ (theorem 1.2 and proposition 2.12(ii))

$\succ(\psi \star \sigma \nu \cdot \pi, p \wedge(q \wedge r))$ (proposition [2.8) $\equiv(\psi, p) \star(\sigma \nu, q) \cdot(\pi, r)$.

Suppose first that $(\psi, p) \|-\top \rightarrow \perp$; then, we have $(\psi, p) \star(\sigma \nu, q) \cdot(\pi, r) \in \mathbb{\Perp}$ and thus:

$(S, \mathbf{1}) \star(\psi, p) \cdot(\nu, q) \cdot(\pi, r) \in \mathbb{\Perp}$. This shows that $(S, \mathbf{1}) \| \Vdash \top \rightarrow \perp, \top \rightarrow \perp$.

Moreover, if we put $\nu=\underline{n}$, so that $\sigma \nu=\underline{n+1}$, and $q=\mathbf{1}$, we have shown that:

$(S, \mathbf{1}) \star(\psi, p) \cdot(\underline{n}, \mathbf{1}) \cdot(\pi, r) \succ(\psi, p) \star(\underline{n+1}, \mathbf{1}) \cdot(\pi, r)$.

Now, we put $T=\lambda f \lambda x\left(\bar{\gamma}^{\prime} x\right) S f \underline{0}$, with $\left.\gamma^{\prime}:: \mathbf{1} \wedge(p \wedge(q \wedge r))\right] \Rightarrow q \wedge(\mathbf{1} \wedge(p \wedge(\mathbf{1} \wedge r)))$.

Then, we have $(T, \mathbf{1}) \star(\phi, p) \cdot(\nu, q) \cdot(\pi, r) \equiv(T \star \phi \cdot \nu \cdot \pi, \mathbf{1} \wedge(p \wedge(q \wedge r))) \succ$

$\left(\bar{\gamma}^{\prime} \nu \star S \cdot \phi \cdot \underline{0} \cdot \pi, \mathbf{1} \wedge(p \wedge(q \wedge r))\right)$ (theorem 1.2 and proposition 2.12(ii))

$\succ(\nu \star S \cdot \phi \cdot \underline{0} \cdot \pi, q \wedge(\mathbf{1} \wedge(p \wedge(\mathbf{1} \wedge r))))$ (proposition 2.8)

$\equiv(\nu, q) \star(S, \mathbf{1}) \cdot(\phi, p) \cdot(\underline{0}, \mathbf{1}) \cdot(\pi, r)$ which is the desired result.

ii) We are looking for a proof-like term $J$ such that $(J, \mathbf{1}) \| \forall \forall x(\operatorname{ent}(x) \rightarrow \operatorname{int}(x))$. It is sufficient to have $(J, \mathbf{1}) \|-\operatorname{ent}\left(s^{n} 0\right) \rightarrow \operatorname{int}\left(s^{n} 0\right)$ for each $n \in \mathbb{N}$, since $|\operatorname{ent}(p)|=\emptyset$ if $p \notin \mathbb{N}$. Let $(\pi, q) \in|\|\operatorname{int}(n) \mid\|$; we must have $(J, \mathbf{1}) \star(\underline{n}, \mathbf{1}) \cdot(\pi, q) \in \mathbb{\Perp}$, that is:

$(J \star \underline{n} \cdot \pi, \mathbf{1} \wedge(\mathbf{1} \wedge q)) \in \mathbb{\Perp}$.

But, we have $\left(\underline{n}^{*}, \mathbf{1}_{\underline{n}}\right)=\left((\sigma)^{n} \underline{0}\right)_{\mathcal{B}} \| \Vdash \operatorname{int}\left(s^{n} 0\right)$ (theorem 1.3, applied in $\mathcal{B}$ ) and therefore: $\left(\underline{n}^{*}, \mathbf{1}_{\underline{n}}\right) \star(\pi, q) \in \mathbb{\Perp}$ or else $\left(\underline{n}^{*} \star \pi, \mathbf{1}_{\underline{n}} \wedge q\right) \in \mathbb{\Perp}$.

Thus, let $\tau \in \mathbf{C}[\mathbf{1} \wedge(\mathbf{1} \wedge q)]$; we have then $(\gamma)^{n}\left(\gamma_{0}\right) \tau \in \mathbf{C}\left[\mathbf{1}_{n} \wedge q\right]$ where $\gamma_{0}$ and $\gamma$ are two $\mathbf{C}$-expressions such that:

$\gamma_{0}:: \mathbf{1} \wedge(\mathbf{1} \wedge q) \Rightarrow(\mathbf{1} \wedge \mathbf{1}) \wedge q ; \gamma:: p \wedge q \Rightarrow\left(\mathbf{1}_{\sigma} \wedge p\right) \wedge q$.

Indeed, we have seen that $\mathbf{1}_{\underline{0}}=\mathbf{1} \wedge \mathbf{1}$ and $\mathbf{1}_{n+1}=\mathbf{1}_{\sigma} \wedge \mathbf{1}_{\underline{n}}$. It follows that, if $\tau \in \mathrm{C}[\mathbf{1} \wedge(\mathbf{1} \wedge q)]$, then $\left(\gamma_{0}\right) \tau \in \mathbf{C}\left[\mathbf{1}_{\underline{0}} \wedge q\right]$, and therefore $(\gamma)^{n} \overline{\left(\gamma_{0}\right)} \tau \in \mathbf{C}\left[\mathbf{1}_{n} \wedge q\right]$.

Thus, we have $\underline{n}^{*} \star \pi^{(\gamma)^{n}\left(\gamma_{0}\right) \tau} \in \mathbb{\Perp}$.

Now, we build below two proof-like terms $g, j$ such that, for each $n \in \mathbb{N}$, we have:

a) $g \star \underline{n} \cdot \xi \cdot \pi^{\tau} \succ \xi \star \pi^{(\gamma)^{n}\left(\gamma_{0}\right) \tau}$

b) $j \star \underline{n} \cdot \xi \cdot \pi \succ \xi \star \underline{n}^{*} \cdot \pi$.

Then, by putting $\bar{J}=\lambda x(g x)(j) x$, we have $J \star \underline{n} \cdot \pi^{\tau} \succ \underline{n}^{*} \star \pi^{(\gamma)^{n}\left(\gamma_{0}\right) \tau} \in \Perp$, which is the desired result.

a) We put $g=\lambda k \lambda x\left(\bar{\gamma}_{0}\right)(k) \bar{\gamma} x$; from theorem 1.2, we have:

$g \star \underline{n} \cdot \xi \cdot \pi^{\tau} \succ \bar{\gamma}_{0} \star(\underline{n}) \bar{\gamma} \xi \cdot \pi^{\tau} \succ(\underline{n}) \bar{\gamma} \xi \star \pi^{\left(\gamma_{0}\right) \tau}$.

Thus, it suffices to show that $(\underline{n}) \bar{\gamma} \xi \star \pi^{\tau} \succ \xi \star \pi^{(\gamma)^{n} \tau}$ which we do by recurrence on $n$.

If $n=0$, we have immediately $\underline{0} \star \bar{\gamma} \cdot \xi \cdot \pi^{\tau} \succ \xi \star \pi^{\tau}$ since $\underline{0}=\lambda x \lambda y y$. 
Going from $n$ to $n+1$ : we have $(\underline{n+1}) \bar{\gamma} \xi \star \pi^{\tau} \equiv(\sigma \underline{n}) \bar{\gamma} \xi \star \pi^{\tau} \succ \sigma \star \underline{n} \cdot \bar{\gamma} \cdot \xi \cdot \pi^{\tau}$ $\succ \bar{\gamma} \star(\underline{n}) \bar{\gamma} \xi \cdot \pi^{\tau} \succ(\underline{n}) \bar{\gamma} \xi \star \pi^{(\gamma) \tau} \succ \overline{\xi \star \pi^{(\gamma)^{n+1} \tau}}$ by induction hypothesis.

b) We put $\beta=\bar{\alpha}_{0} \sigma^{*}, U=\lambda g \lambda y(g)(\beta) y$ and $j=\lambda k \lambda f(k) U f \underline{0}^{*}$.

Therefore, we have $j \star \underline{n} \cdot \xi \cdot \pi \succ \underline{n} U \xi \star \underline{0}^{*} \cdot \pi$. We show, by recurrence on $n$, that:

$\underline{n} U \xi \star \underline{k}^{*} \cdot \pi \succ \xi \star(\underline{n+k})^{*} \cdot \pi$ for each integer $k$, which gives the desired result with $k=0$.

For $n=0$, we have $\underline{0 U \xi} \star \underline{k}^{*} \cdot \pi \succ \xi \star \underline{k}^{*} \cdot \pi$ since $\underline{0}=\lambda x \lambda y y$.

Going from $n$ to $n+1$ : we have $(\underline{n+1}) \star U \cdot \xi \cdot \underline{k}^{*} \cdot \pi \equiv \sigma \underline{n} \star U \cdot \xi \cdot \underline{k}^{*} \cdot \pi \succ U \star \underline{n} U \xi \cdot \underline{k}^{*} \cdot \pi$

$($ since $\sigma=\lambda n \lambda f \lambda x(f)(n) f x) \succ \underline{n} U \xi \star \beta \underline{k}^{*} \cdot \pi \equiv \underline{n} U \xi \star(\underline{k+1})^{*} \cdot \pi \succ \xi \star(\underline{n+k+1})^{*} \cdot \pi$

by induction hypothesis.

\section{ForCING}

Forcing is a method to compute truth values of formulas in the generic $\mathcal{B}$-model $\mathcal{N}$.

For each $k$-ary predicate variable $X$, we add to the language a new predicate variable, denoted by $X^{+}$, which has arity $k+1$. In the $\mathcal{A}$-model $\mathcal{M}$, we use the variables $X$ and $X^{+}$; in the $\mathcal{B}$-model $\mathcal{N}$, only the variables $X$.

With each $k$-ary second order parameter $\mathcal{X}: P^{k} \rightarrow \mathcal{P}(\boldsymbol{\Pi})$ of the model $\mathcal{N}$, we associate a $(k+1)$-ary second order parameter $\mathcal{X}^{+}: P^{k+1} \rightarrow \mathcal{P}(\Pi)$ of the model $\mathcal{M}$. It is defined in an obvious way, since $\Pi=\Pi \times P$; we put:

$\mathcal{X}^{+}\left(p, p_{1}, \ldots, p_{k}\right)=\left\{\pi \in \Pi ;(\pi, p) \in \mathcal{X}\left(p_{1}, \ldots, p_{k}\right)\right\}$.

For each formula $F$ written without the variables $X^{+}$, with parameters in the model $\mathcal{N}$, we define, by recurrence on $F$, a formula denoted by $p \Vdash F$ (read " $p$ forces $F$ "), with parameters in the model $\mathcal{A}$, written with the variables $X^{+}$and a free condition variable $p$ :

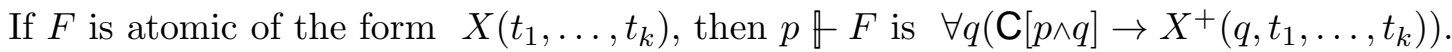

If $F$ is atomic of the form $\mathcal{X}\left(t_{1}, \ldots, t_{k}\right)$, then $p \mathbb{F} F$ is $\forall q\left(\mathrm{C}[p \wedge q] \rightarrow \mathcal{X}^{+}\left(q, t_{1}, \ldots, t_{k}\right)\right)$.

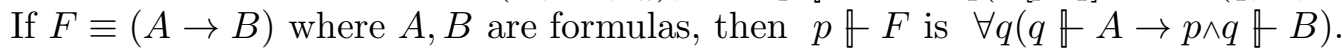

If $F \equiv\left(\mathrm{R}\left(t_{1}, \ldots, t_{k}\right) \rightarrow B\right)$, where $\mathrm{R}$ is a predicate constant, then:

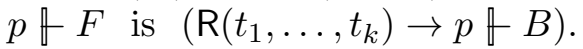

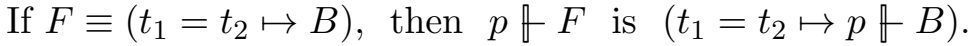

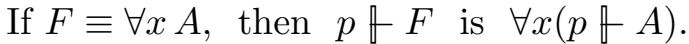

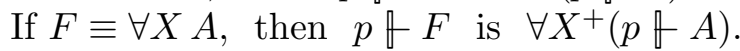

Thus we have, in particular:

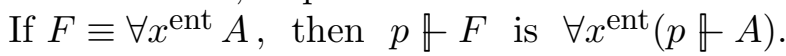

Lemma 3.1. Let $F$ be a formula the free variables of which are amongst $X_{1}, \ldots, X_{k}$ and let $\mathcal{X}_{1}, \ldots, \mathcal{X}_{k}$ be second order parameters in the model $\mathcal{N}$, with corresponding arities. Then,

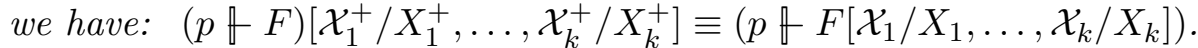

Proof. Immediate, by recurrence on $F$.

\section{Theorem 3.2.}

For each closed formula $F$ with parameters in the model $\mathcal{N}$, there exist two proof-like terms $\chi_{F}, \chi_{F}^{\prime}$, which only depend on the propositional structure of $F$, such that we have:

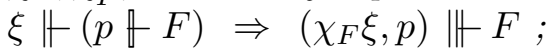

$(\xi, p) \| F \Rightarrow \chi_{F}^{\prime} \xi \Vdash(p \mathbb{R} F)$

for every $\xi \in \Lambda$ and $p \in P$. 
The propositional structure of $F$ is the simple type built with only one atom $O$ and the connective $\rightarrow$, which is obtained from $F$ by deleting all quantifiers, all symbols $\mapsto$ with their hypothesis, and by identifying all atomic formulas with $O$.

For instance, the propositional structure of the formula:

$\forall X(\forall x(\forall y(f(x, y)=0 \mapsto X y) \rightarrow X x) \rightarrow \forall x X x)$ is $(O \rightarrow O) \rightarrow O$.

Proof. By recurrence on the length of $F$.

- If $F$ is atomic, we have $F \equiv \mathcal{X}\left(t_{1}, \ldots, t_{k}\right)$; we show that $\chi_{F}=\chi$ and $\chi_{F}^{\prime}=\chi^{\prime}$.

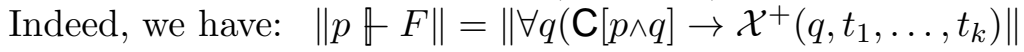

$$
=\bigcup_{q}\left\{\tau \cdot \pi ; \tau \in \mathrm{C}[p \wedge q],(\pi, q) \in\left\|\mathcal{X}\left(t_{1}, \ldots, t_{k}\right) \mid\right\|\right\}
$$

because, by definition of $\mathcal{X}^{+}$, we have $\pi \in\left\|\mathcal{X}^{+}\left(q, t_{1}, \ldots, t_{k}\right)\right\| \Leftrightarrow(\pi, q) \in\left\|\mathcal{X}\left(t_{1}, \ldots, t_{k}\right)\right\|$.

Therefore, we have:

$(*) \quad \xi \Vdash(p \mathbb{F}) \Leftrightarrow$

$$
(\forall q \in P)(\forall \tau \in \mathrm{C}[p \wedge q])(\forall \pi \in \Pi)\left((\pi, q) \in\left\|\mathcal{X}\left(t_{1}, \ldots, t_{k}\right)\right\| \Rightarrow \xi \star \tau \cdot \pi \in \mathbb{\Perp}\right) .
$$

Moreover, we have $(\xi, p) \| \vdash F \Leftrightarrow(\forall q \in P)(\forall \pi \in \Pi)((\pi, q) \in\|F\| \Rightarrow(\xi, p) \star(\pi, q) \in \mathbb{\Perp})$

$\Leftrightarrow(\forall q \in P)(\forall \pi \in \Pi)((\pi, q) \in\|F \mid\| \Rightarrow(\xi \star \pi, p \wedge q) \in \mathbb{L})$ and finally, by definition of $\mathbb{L}$ :

$(* *) \quad(\xi, p) \| \models F \Leftrightarrow(\forall q \in P)(\forall \tau \in \mathrm{C}[p \wedge q])(\forall \pi \in \Pi)\left((\pi, q) \in\|F\| \Rightarrow \xi \star \pi^{\tau} \in \Perp\right)$.

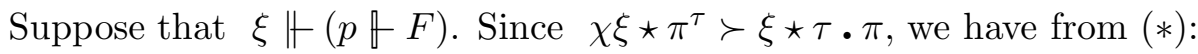

$(\forall q \in P)(\forall \tau \in \mathrm{C}[p \wedge q])(\forall \pi \in \Pi)\left((\pi, q) \in\left\|\mathcal{X}\left(t_{1}, \ldots, t_{k}\right)\right\| \| \chi \xi \star \tau \cdot \pi \in \mathbb{\Perp}\right)$

and therefore $(\chi \xi, p) \| \mapsto F$ from $(* *)$.

Conversely, suppose that $(\xi, p) \| F$. By applying $(* *)$ and $\chi^{\prime} \xi \star \tau \cdot \pi \succ \xi \star \pi^{\tau}$, we obtain

$(\forall q \in P)(\forall \tau \in \mathrm{C}[p \wedge q])(\forall \pi \in \Pi)\left((\pi, q) \in\|\mid\| F\|\| \chi^{\prime} \xi \star \tau \cdot \pi \in \mathbb{\Perp}\right)$

and therefore $\chi^{\prime} \xi \Vdash(p \mathbb{F})$ from $(*)$.

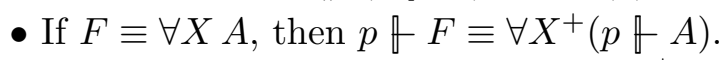

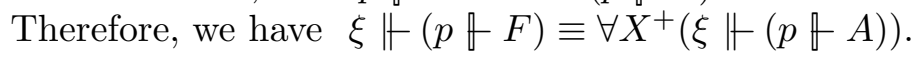

Moreover, we have $(\xi, p) \|-F \equiv \forall X((\xi, p) \|-A)$.

Let $\mathcal{X}: P^{k} \rightarrow \mathcal{P}(\boldsymbol{\Pi})$ be a second order parameter in the model $\mathcal{N}$, with the same arity as $X$, and let $\mathcal{X}^{+}$be the corresponding parameter of the model $\mathcal{M}$.

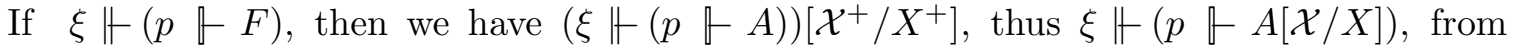
lemma 3.1 .

By the recurrence hypothesis, we have $\left(\chi_{A} \xi, p\right) \| A[\mathcal{X} / X]$. Since $\mathcal{X}$ is arbitrary, it follows that $\left(\chi_{A} \xi, p\right) \| \forall \forall X$.

Conversely, if we have $(\xi, p) \| \Vdash F$, then $(\xi, p) \| \mapsto A[\mathcal{X} / X]$ for every $\mathcal{X}$.

By the recurrence hypothesis, we have $\chi_{A}^{\prime} \xi \Vdash(p \llbracket A[\mathcal{X} / X])$, and therefore:

$\left.\chi_{A}^{\prime} \xi \Vdash(p \nVdash A)\left[\mathcal{X}^{+} / X^{+}\right]\right)$, from lemma 3.1. Since $\mathcal{X}^{+}$is arbitrary, it follows that:

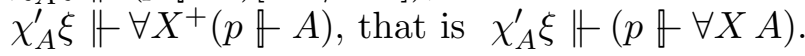

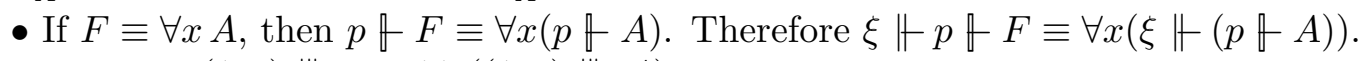

Moreover, $(\xi, p) \|-F \equiv \forall x((\xi, p) \|-A)$.

The result is immediate, from the recurrence hypothesis.

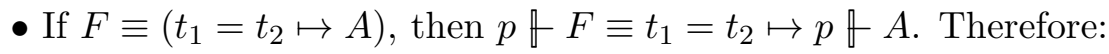

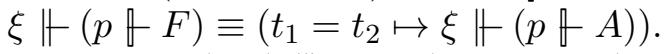

Moreover, $(\xi, p) \|-F \equiv\left(t_{1}=t_{2} \mapsto(\xi, p) \|-A\right)$.

The result is immediate, from the recurrence hypothesis.

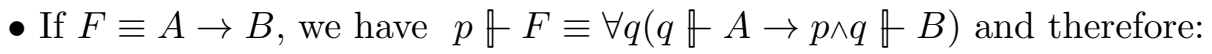

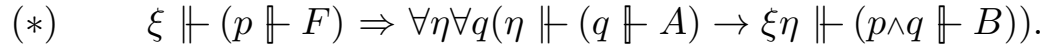

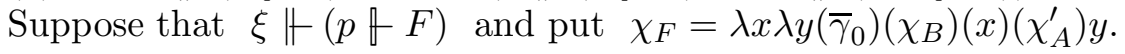


We must show $\left(\chi_{F} \xi, p\right) \| \mapsto A \rightarrow B$; thus, let $(\eta, q) \| \Vdash A$ and $(\pi, r) \in\|B\|$.

We must show $\left(\chi_{F} \xi, p\right) \star(\eta, q) \cdot(\pi, r) \in \mathbb{\Perp}$ that is $\left(\chi_{F} \xi \star \eta \cdot \pi, p \wedge(q \wedge r)\right) \in \mathbb{\Perp}$.

Thus, let $\tau \in \mathrm{C}[p \wedge(q \wedge r)]$; we must show $\chi_{F} \xi \star \eta \cdot \pi^{\tau} \in \Perp$ or else $\chi_{F} \star \xi \cdot \eta \cdot \pi^{\tau} \in \Perp$.

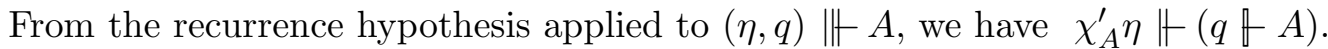

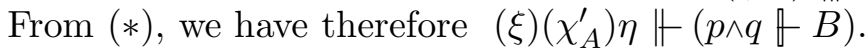

Applying again the recurrence hypothesis, we get:

$\left(\left(\chi_{B}\right)(\xi)\left(\chi_{A}^{\prime}\right) \eta, p \wedge q\right) \| \leftarrow B$. But since $(\pi, r) \in\|B\|$, we have:

$\left(\left(\chi_{B}\right)(\xi)\left(\chi_{A}^{\prime}\right) \eta, p \wedge q\right) \star(\pi, r) \in \mathbb{\Perp}$, that is $\left(\left(\chi_{B}\right)(\xi)\left(\chi_{A}^{\prime}\right) \eta \star \pi,(p \wedge q) \wedge r\right) \in \mathbb{\Perp}$.

Since $\tau \in \mathbf{C}[p \wedge(q \wedge r)]$, we have $\gamma_{0} \tau \in \mathbf{C}[(p \wedge q) \wedge r]$ and therefore $\left(\chi_{B}\right)(\xi)\left(\chi_{A}^{\prime}\right) \eta \star \pi^{\gamma_{0} \tau} \in \Perp$.

But, by definition of $\chi_{F}$, we have, from theorem 1.2.

$\chi_{F} \star \xi \cdot \eta \cdot \pi^{\tau} \succ\left(\chi_{B}\right)(\xi)\left(\chi_{A}^{\prime}\right) \eta \star \pi^{\gamma_{0} \tau}$ which gives the desired result: $\chi_{F} \star \xi \cdot \eta \cdot \pi^{\tau} \in \mathbb{\Perp}$.

Suppose now that $(\xi, p) \| \mapsto A \rightarrow B$; we put $\chi_{F}^{\prime}=\lambda x \lambda y\left(\chi_{B}^{\prime}\right)\left(\bar{\alpha}_{0} x\right)\left(\chi_{A}\right) y$.

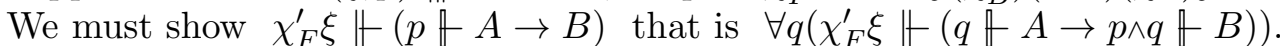

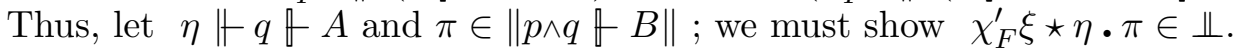

By the recurrence hypothesis, we have $\left(\chi_{A} \eta, q\right) \| A$, therefore $(\xi, p)\left(\chi_{A} \eta, q\right) \| \rightarrow B$ or else, by definition of the algebra $\mathcal{B}:\left(\left(\bar{\alpha}_{0} \xi\right)\left(\chi_{A}\right) \eta, p \wedge q\right) \| \leftarrow B$.

Applying again the recurrence hypothesis, we have $\left(\chi_{B}^{\prime}\right)\left(\bar{\alpha}_{0} \xi\right)\left(\chi_{A}\right) \eta \Vdash(p \wedge q \mathbb{} \mathbb{B})$ and therefore:

$\left(\chi_{B}^{\prime}\right)\left(\bar{\alpha}_{0} \xi\right)\left(\chi_{A}\right) \eta \star \pi \in \Perp$. But we have:

$\chi_{F}^{\prime} \xi \star \eta \cdot \pi \succ \chi_{F}^{\prime} \star \xi \cdot \eta \cdot \pi \succ\left(\chi_{B}^{\prime}\right)\left(\bar{\alpha}_{0} \xi\right)\left(\chi_{A}\right) \eta \star \pi$ from theorem 1.2 ; the desired result follows.

A formula $F$ is said to be first order if it is obtained by the following rules:

- $\perp$ is first order.

- If $A, B$ are first order, then $A \rightarrow B$ is first order.

- If $B$ is first order, $\mathrm{R}$ is a predicate symbol and $t_{1}, \ldots, t_{k}$ are terms with parameters, then $\mathrm{R}\left(t_{1}, \ldots, t_{k}\right) \rightarrow B, t_{1}=t_{2} \mapsto B$ are first order.

- If $A$ is first order, then $\forall x A$ is first order ( $x$ is an individual variable).

Remarks.

i) If $A$ is a first order formula, it is the same for $\forall x^{\text {ent }} A$.

ii) This notion will be extended below (see proposition 4.3) .

Theorem 3.3. Let $F$ be a closed first order formula. There exist two proof-like terms $\delta_{F}, \delta_{F}^{\prime}$, which depend only on the propositional structure of $F$, such that we have:

$\xi \Vdash(\mathrm{C}[p] \rightarrow F) \Rightarrow\left(\delta_{F} \xi, p\right) \| \nVdash F$

$(\xi, p) \| F \Rightarrow \delta_{F}^{\prime} \xi \Vdash(\mathrm{C}[p] \rightarrow F)$

for every $\xi \in \Lambda$ and $p \in P$.

Proof. The proof is by recurrence on the construction of $F$ following the above rules.

- If $F$ is $\perp$, we put:

$\delta_{\perp}=\lambda x(\chi) \lambda y(x)(\alpha) y$ with $\alpha:: p \wedge q \Rightarrow p$.

$\delta_{\perp}^{\prime}=\lambda x \lambda y\left(\chi^{\prime} x\right)\left(\alpha^{\prime}\right) y$ with $\alpha^{\prime}:: p \Rightarrow p \wedge \mathbf{1}$

Indeed, suppose that $\xi \| \mathrm{C}[p] \rightarrow \perp$ and let us show that $\left(\delta_{\perp} \xi, p\right)(\pi, q) \in \mathbb{\Perp}$, that is:

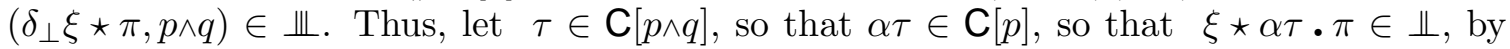
hypothesis on $\xi$, which gives $\delta_{\perp} \xi \star \pi^{\tau} \in \Perp \Perp$.

Conversely, if $(\xi, p) \| \Perp \perp$, we have $(\xi, p) \star(\pi, \mathbf{1}) \equiv(\xi \star \pi, p \wedge \mathbf{1}) \in \mathbb{\Perp}$ for every $\pi \in \Pi$. But, if $\tau \in \mathrm{C}[p]$, we have $\alpha^{\prime} \tau \in \mathrm{C}[p \wedge \mathbf{1}]$, therefore $\xi \star \pi^{\alpha^{\prime} \tau} \in \Perp \Perp \Perp$, thus $\delta_{\perp}^{\prime} \xi \star \tau \cdot \pi \in \Perp \Perp \Perp$. Therefore $\delta_{\perp}^{\prime} \xi \Vdash \mathrm{C}[p] \rightarrow \perp$. 
- If $F$ is $A \rightarrow B$, we put:

$\delta_{A \rightarrow B}=\lambda x \lambda y(\chi) \lambda z\left(\left(\chi^{\prime}\right)\left(\delta_{B}\right) \lambda d((x)(\alpha) z)\left(\delta_{A}^{\prime} y\right)(\beta) z\right)(\gamma) z$ with

$\alpha:: p \wedge(q \wedge r) \Rightarrow p ; \beta:: p \wedge(q \wedge r) \Rightarrow q ; \gamma:: p \wedge(q \wedge r) \Rightarrow \mathbf{1} \wedge r$.

Indeed, suppose that $\xi\|\mathrm{C}[p], A \rightarrow B,(\eta, q)\| \leftarrow A$ and $(\pi, r) \in\|B\|$.

We must show $\left(\delta_{A \rightarrow B} \xi, p\right) \star(\eta, q) \cdot(\pi, r) \in \mathbb{\Perp}$, that is $\left(\delta_{A \rightarrow B} \xi \star \eta \bullet \pi, p \wedge(q \wedge r)\right) \in \mathbb{\Perp}$.

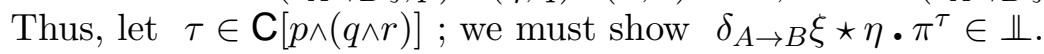

We have $\alpha \tau \in \mathrm{C}[p], \beta \tau \in \mathrm{C}[q]$; but, by the recurrence hypothesis, we have:

$\delta_{A}^{\prime} \eta \Vdash \mathrm{C}[q] \rightarrow A$, therefore $\left(\delta_{A}^{\prime} \eta\right)(\beta) \tau \Vdash A$ and $((\xi)(\alpha) \tau)\left(\delta_{A}^{\prime} \eta\right)(\beta) \tau \Vdash B$;

thus $\lambda d((\xi)(\alpha) \tau)\left(\delta_{A}^{\prime} \eta\right)(\beta) \tau \Vdash \mathbf{C}[\mathbf{1}] \rightarrow B$.

From the recurrence hypothesis, we have $\left(\left(\delta_{B}\right) \lambda d((\xi)(\alpha) \tau)\left(\delta_{A}^{\prime} \eta\right)(\beta) \tau, \mathbf{1}\right) \| \mapsto B$, thus:

$\left(\left(\delta_{B}\right) \lambda d((\xi)(\alpha) \tau)\left(\delta_{A}^{\prime} \eta\right)(\beta) \tau, \mathbf{1}\right) \star(\pi, r) \in \mathbb{H}$, that is:

$\left(\left(\delta_{B}\right) \lambda d((\xi)(\alpha) \tau)\left(\delta_{A}^{\prime} \eta\right)(\beta) \tau \star \pi, \mathbf{1} \wedge r\right) \in \mathbb{\Perp}$.

But, we have $\gamma \tau \in \mathrm{C}[\mathbf{1} \wedge r]$, therefore $\left(\delta_{B}\right) \lambda d((\xi)(\alpha) \tau)\left(\delta_{A}^{\prime} \eta\right)(\beta) \tau \star \pi^{\gamma \tau} \in \Perp \Perp$, and thus:

$\left(\left(\chi^{\prime}\right)\left(\delta_{B}\right) \lambda d((\xi)(\alpha) \tau)\left(\delta_{A}^{\prime} \eta\right)(\beta) \tau\right)(\gamma) \tau \star \pi \in \mathbb{\Perp}$. It follows that:

$(\chi) \lambda z\left(\left(\chi^{\prime}\right)\left(\delta_{B}\right) \lambda d((\xi)(\alpha) z)\left(\delta_{A}^{\prime} \eta\right)(\beta) z\right)(\gamma) z \star \pi^{\tau} \in \Perp \Perp$ so that $\delta_{A \rightarrow B} \xi \star \eta \cdot \pi^{\tau} \in \Perp \Perp$.

We now put:

$\delta_{A \rightarrow B}^{\prime}=\lambda x \lambda y \lambda z\left(\left(\delta_{B}^{\prime}\right)\left(\overline{\alpha_{0}} x\right)\left(\delta_{A}\right) \lambda d z\right)(\alpha) y$ with $\alpha:: p \Rightarrow p \wedge \mathbf{1}$.

Suppose that $(\xi, p) \|+A \rightarrow B$; let $\tau \in \mathrm{C}[p], \eta \nVdash A$ and $\pi \in\|B\|$. We must show:

$\delta_{A \rightarrow B}^{\prime} \xi \star \tau \cdot \eta \cdot \pi \in \mathbb{H}$. We have $\lambda d \eta \Vdash \mathrm{C}[\mathbf{1}] \rightarrow A$; applying the recurrence hypothesis, we have $\left(\left(\delta_{A}\right) \lambda d \eta, \mathbf{1}\right) \|-A$, thus $(\xi, p)\left(\left(\delta_{A}\right) \lambda d \eta, \mathbf{1}\right) \|-B$ that is $\left(\left(\overline{\alpha_{0}} \xi\right)\left(\delta_{A}\right) \lambda d \eta, p \wedge \mathbf{1}\right) \|-B$.

Applying again the recurrence hypothesis, we find:

$\left(\delta_{B}^{\prime}\right)\left(\overline{\alpha_{0}} \xi\right)\left(\delta_{A}\right) \lambda d \eta \Vdash \mathrm{C}[p \wedge \mathbf{1}] \rightarrow B$. Since we have $\alpha \tau \in \mathrm{C}[p \wedge \mathbf{1}]$, we get:

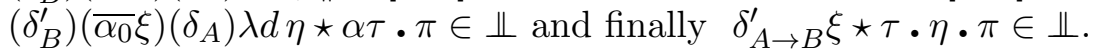

- If $F \equiv \mathrm{R}(\vec{q}) \rightarrow B$, where $\mathrm{R}$ is a $k$-ary predicate symbol and $\vec{p} \in P^{k}$, we put:

$\delta_{R \rightarrow B}=\lambda x \lambda y(\bar{\alpha})\left(\delta_{B}\right) \lambda z(x) z y$ with $\alpha:: p \wedge(\mathbf{1} \wedge r) \Rightarrow p \wedge r$.

$\delta_{R \rightarrow B}^{\prime}=\lambda x \lambda y \lambda z\left(\left(\delta_{B}^{\prime}\right)\left(\bar{\alpha}_{0}\right) x z\right)\left(\alpha^{\prime}\right) y$ with $\alpha^{\prime}:: p \Rightarrow p \wedge \mathbf{1}$.

Suppose that $\xi \| \mathrm{C}[p], \mathrm{R}[\vec{q}] \rightarrow B$ and let $\eta \in|\mathrm{R}[\vec{q}]|,(\pi, r) \in\|B\|$. We must show:

$\left(\delta_{R \rightarrow B} \xi, p\right) \star(\eta, \mathbf{1}) \cdot(\pi, r) \in \mathbb{\Perp}$, that is $\left(\delta_{R \rightarrow B} \xi \star \eta \cdot \pi, p \wedge(\mathbf{1} \wedge r)\right) \in \mathbb{\Perp}$.

Thus, let $\tau \in \mathrm{C}[p \wedge(\mathbf{1} \wedge r)]$; we must show $\delta_{R \rightarrow B} \xi \star \eta \bullet \pi^{\tau} \in \Perp \Perp \Perp l$. But, we have:

$\lambda z(\xi) z \eta \Vdash \mathrm{C}[p] \rightarrow B$, and thus $\left(\left(\delta_{B}\right) \lambda z(\xi) z \eta, p\right) \| \mapsto B$, by the recurrence hypothesis.

It follows that $\left(\left(\delta_{B}\right) \lambda z(\xi) z \eta, p\right) \star(\pi, r) \in \mathbb{\Perp}$, that is:

$\left(\left(\delta_{B}\right) \lambda z(\xi) z \eta \star \pi, p \wedge r\right) \in \mathbb{H}$. But we have $\alpha \tau \in \mathrm{C}[p \wedge r]$, and therefore:

$\left(\delta_{B}\right) \lambda z(\xi) z \eta \star \pi^{\alpha \tau} \in \mathbb{\Perp}$, thus $(\bar{\alpha})\left(\delta_{B}\right) \lambda z(\xi) z \eta \star \pi^{\tau} \in \mathbb{\Perp}$, therefore $\delta_{R \rightarrow B} \xi \star \eta \bullet \pi^{\tau} \in \mathbb{\Perp}$.

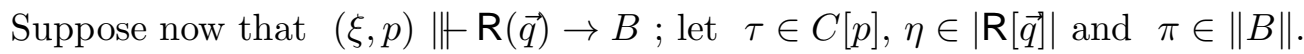

We must show $\delta_{R \rightarrow B}^{\prime} \xi \star \tau \cdot \eta \cdot \pi \in \mathbb{\Perp}$. But, we have $(\xi, p)(\eta, \mathbf{1}) \| \mapsto B$, that is:

$\left(\left(\bar{\alpha}_{0}\right) \xi \eta, p \wedge \mathbf{1}\right) \| \mapsto B$, thus $\left(\delta_{B}^{\prime}\right)\left(\bar{\alpha}_{0}\right) \xi \eta \Vdash \mathrm{C}[p \wedge \mathbf{1}] \rightarrow B$, by recurrence hypothesis.

But, we have $\alpha^{\prime} \tau \in \mathrm{C}[p \wedge \mathbf{1}]$, therefore $\left(\delta_{B}^{\prime}\right)\left(\bar{\alpha}_{0}\right) \xi \eta \star \alpha^{\prime} \tau \cdot \pi \in \Perp \Perp \Perp$, hence the result.

- If $F \equiv\left(p_{1}=p_{2} \mapsto B\right)$, we put $\delta_{F}=\delta_{B}$ and $\delta_{F}^{\prime}=\delta_{B}^{\prime}$.

Indeed, suppose that $\xi \Vdash \mathbf{C}[p] \rightarrow\left(p_{1}=p_{2} \mapsto B\right)$ and $(\pi, q) \in\left\|p_{1}=p_{2} \mapsto B\right\|$. We must show that $\left(\delta_{B} \xi, p\right) \star(\pi, q) \in \mathbb{H}$. Since $\left\|p_{1}=p_{2} \mapsto B\right\| \neq \emptyset$, we have $p_{1}=p_{2}$, thus $(\pi, q) \in\|B\|$ and $\xi \Vdash \mathrm{C}[p] \rightarrow B$. Hence the result, by the recurrence hypothesis.

Suppose now that $(\xi, p)\left\|-p_{1}=p_{2} \mapsto B, \tau\right\| \mathrm{C}[p]$ et $\pi \in\left\|p_{1}=p_{2} \mapsto B\right\|$. We must show $\delta_{B}^{\prime} \star \tau \cdot \pi \in \Perp$. Since $\left\|p_{1}=p_{2} \mapsto B\right\| \neq \emptyset$, we have $p_{1}=p_{2}$, therefore $\pi \in\|B\|$ and

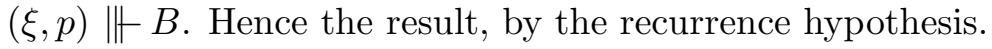

- If $F \equiv \forall x A$, we put $\delta_{F}=\delta_{A}$ and $\delta_{F}^{\prime}=\delta_{A}^{\prime}$. 
Indeed, if $\xi \Vdash \mathrm{C}[p] \rightarrow \forall x A$, we have $\xi \Vdash \mathrm{C}[p] \rightarrow A[a / x]$ for every $a \in P$. By the recurrence hypothesis, we have $\left(\delta_{A} \xi, p\right) \| \leftarrow A[a / x]$; thus $\left(\delta_{A} \xi, p\right) \| \forall \forall x A$.

If $(\xi, p) \| \Vdash \forall x A$, we have $(\xi, p) \| \models A[a / x]$ for every $a \in P$. By the recurrence hypothesis, we have $\delta_{A}^{\prime} \xi \Vdash \mathrm{C}[p] \rightarrow A[a / x]$; thus $\delta_{A}^{\prime} \xi \Vdash \mathrm{C}[p] \rightarrow \forall x A$.

The generic ideal. We define a unary predicate $\mathcal{J}: P \rightarrow \mathcal{P}(\boldsymbol{\Pi})$ in the model $\mathcal{N}$ (second order parameter of arity 1), by putting $\mathcal{J}(p)=\Pi \times\{p\}$; we call it the generic ideal.

Thus, the binary predicate $\mathcal{J}^{+}: P^{2} \rightarrow \mathcal{P}(\Pi)$ which corresponds to it in the model $\mathcal{M}$, is such that $\mathcal{J}^{+}(p, q)=\emptyset$ (resp. $\left.\Pi\right)$ if $p \neq q$ (resp. $p=q$ ). In other words:

$\mathcal{J}^{+}(p, q)$ is the predicate $p \neq q$.

The formula $p \nVdash \mathcal{J}(q)$ is $\forall r\left(\mathrm{C}[p \wedge r] \rightarrow \mathcal{J}^{+}(r, q)\right)$. Therefore, we have:

$\|p \Vdash \mathcal{J}(q)\|=\|\neg \mathrm{C}[p \wedge q]\|$; in other words:

$$
p \Vdash \mathcal{J}(q) \text { is exactly } \neg \mathrm{C}[p \wedge q] .
$$

\section{Notations.}

- We denote by $p \sqsubseteq q$ the formula $\forall r(\neg \mathrm{C}[q \wedge r] \rightarrow \neg \mathrm{C}[p \wedge r])$ and by $p \sim q$ the formula $p \sqsubseteq q \wedge q \sqsubseteq p$, that is $\forall r(\neg \mathrm{C}[q \wedge r] \leftrightarrow \neg \mathrm{C}[p \wedge r])$.

In the sequel, we shall often write $F \rightarrow \mathrm{C}[p]$ instead of $\neg \mathrm{C}[p] \rightarrow \neg F$;

Then $p \sqsubseteq q$ is written $\forall r(\mathrm{C}[p \wedge r] \rightarrow \mathrm{C}[q \wedge r])$ and $p \sim q$ is written $\forall r(\mathrm{C}[p \wedge r] \leftrightarrow \mathrm{C}[q \wedge r])$.

Remark. We recall that $\mathrm{C}[p]$ is not a formula, but a subset of $\Lambda$; in fact, in some realizability models which will be considered below, there will exist a formula $\mathbb{C}[p]$ such that:

$|\mathrm{C}[p]|=\left\{\tau \in \Lambda_{c} ; \tau \| \mathbb{C}[p]\right\}$. In such cases, we can identify $\mathbb{C}[p]$ with the formula $\mathbb{C}[p]$.

- If $F$ is a closed formula, we shall write $\| \models F$ to mean that there exists a proof-like term $\theta$ such that $(\theta, \mathbf{1}) \|-F$. From proposition 2.12(i), this is equivalent to say that there exists a proof-like term $\theta$ such that $(\theta, p) \| \mapsto F$ for every $p \in P$.

\section{Proposition 3.4.}

i) $\xi \Vdash \neg \mathrm{C}[p \wedge q] \Rightarrow(\chi \xi, p) \| \leftarrow \mathcal{J}(q)$; $(\xi, p) \|-\mathcal{J}(q) \Rightarrow \chi^{\prime} \xi \Vdash \neg \mathrm{C}[p \wedge q]$.

ii) $\xi \Vdash \forall r(\mathrm{C}[p \wedge(\mathbf{1} \wedge r)], \mathrm{C}[q] \rightarrow \perp) \Rightarrow(\chi \xi, p) \| \neg \mathrm{C}[q]$; $(\xi, p) \| \dashv \neg[q] \Rightarrow \chi^{\prime} \xi \Vdash \forall r(\mathrm{C}[p \wedge(\mathbf{1} \wedge r)], \mathrm{C}[q] \rightarrow \perp)$.

iii) If $\xi \Vdash \neg \mathrm{R}\left(a_{1}, \ldots, a_{k}\right)$ then $(\xi, p) \| \neg \mathrm{R}\left(a_{1}, \ldots, a_{k}\right)$ for all $p$

( $\mathrm{R}$ is a predicate symbol of arity $k$ ).

\section{Proof.}

i) If $\xi \Vdash \neg \mathrm{C}[p \wedge q]$, then $\xi \star \tau \cdot \pi \in \Perp$ and therefore $\chi \xi \star \pi^{\tau} \in \Perp$ for all $\tau \in \mathrm{C}[p \wedge q]$. Thus, we

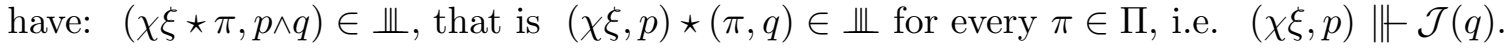
If $(\xi, p) \| \mathcal{J}[q]$, we have $(\xi, p) \star(\pi, q) \in \mathbb{\Perp}$, thus $(\xi \star \pi, p \wedge q) \in \mathbb{\Perp}$ for all $\pi \in \Pi$. Therefore, we have $\xi \star \pi^{\tau} \in \Perp$, that is $\chi^{\prime} \xi \star \tau \cdot \pi \in \Perp$ for each $\tau \in \mathbf{C}[p \wedge q]$. Therefore $\chi^{\prime} \xi \Vdash \neg \mathrm{C}[p \wedge q]$. ii) If $\xi \Vdash \forall r(\mathrm{C}[p \wedge(\mathbf{1} \wedge r)], \mathrm{C}[q] \rightarrow \perp)$, we have $\xi \star v \cdot \tau \cdot \pi \in \Perp$ if $v \in \mathrm{C}[p \wedge(\mathbf{1} \wedge r)]$ and $\tau \in \mathrm{C}[q]$. Therefore $\chi \xi \star \tau \cdot \pi^{v} \in \Perp \Perp \Perp$, thus $(\chi \xi \star \tau \cdot \pi, p \wedge(\mathbf{1} \wedge r)) \in \Perp \Perp \Perp$ that is:

$(\chi \xi, p) \star(\tau, \mathbf{1}) \cdot(\pi, r) \in \Perp$. But $(\tau, \mathbf{1})$ is arbitrary in $\mathbf{C}_{\mathcal{N}}[q]$, and therefore:

$(\chi \xi, p) \| \vdash \mathrm{C}[q] \rightarrow \perp$.

If $(\xi, p) \| \neg \mathrm{C}[q]$, we have $(\xi, p) \star(\tau, \mathbf{1}) \cdot(\pi, r) \in \mathbb{\Perp}$, and therefore $(\xi \star \tau \cdot \pi, p \wedge(\mathbf{1} \wedge r)) \in \mathbb{\Perp}$ for each $\tau \in \mathrm{C}[q]$. Thus, we have $\xi \star \tau \cdot \pi^{v} \in \Perp$ therefore $\chi^{\prime} \xi \star v \cdot \tau \cdot \pi \in \Perp$ for each $v \in \mathrm{C}[p \wedge(\mathbf{1} \wedge r)]$. It follows that $\chi^{\prime} \xi \Vdash \forall r(\mathrm{C}[p \wedge(\mathbf{1} \wedge r)], \mathrm{C}[q] \rightarrow \perp)$.

iii) Let $\tau \in\left|\mathrm{R}\left(a_{1}, \ldots, a_{k}\right)\right|$; we have $\xi \star \tau \cdot \pi \in \Perp \Perp \Perp$ for all $\pi \in \Pi$, thus $(\xi \star \tau \cdot \pi, a) \in \mathbb{\Perp}$ for all $a \in P$, and therefore $(\xi, p) \star(\tau, \mathbf{1}) \cdot(\pi, q) \in \mathbb{\Perp}$. 
Theorem 3.5 (Elementary properties of the generic ideal).

i) $(\bar{\alpha}, \mathbf{1}) \| \vdash \neg \mathcal{J}(\mathbf{1})$ with $\alpha:: \mathbf{1} \wedge(p \wedge q) \Rightarrow p \wedge \mathbf{1}$.

ii) $(\theta, \mathbf{1}) \| \forall \forall x(\neg \mathbf{C}[x] \rightarrow \mathcal{J}(x))$ where $\theta=\lambda x(\chi) \lambda y\left(\left(\chi^{\prime} x\right)(\beta) y\right)(\alpha) y$

with $\alpha:: \mathbf{1} \wedge(p \wedge q) \Rightarrow q$ and $\beta:: \mathbf{1} \wedge(p \wedge q) \Rightarrow p \wedge(\mathbf{1} \wedge \mathbf{1})$.

iii) $(\theta, \mathbf{1}) \| \mapsto \forall x \forall y(\mathcal{J}(x \wedge y), \neg \mathcal{J}(x) \rightarrow \mathcal{J}(y))$ where $\theta=\lambda x \lambda y(\bar{\alpha})(y)(\bar{\beta}) x \quad$ with $\quad \alpha \quad::$ $\mathbf{1} \wedge\left(p^{\prime} \wedge\left(q^{\prime} \wedge q\right)\right) \Rightarrow q^{\prime} \wedge\left(\left(q \wedge p^{\prime}\right) \wedge \mathbf{1}\right)$ and $\beta::\left(q \wedge p^{\prime}\right) \wedge p \Rightarrow p^{\prime} \wedge(p \wedge q)$.

iv) $(\theta, \mathbf{1}) \| \forall \forall x(\forall y(\neg \mathrm{C}[x \wedge y] \rightarrow \mathcal{J}(y)) \rightarrow \neg \mathcal{J}(x))$ where $\theta=\lambda x \lambda y(\bar{\gamma})(x) \lambda z\left(\chi^{\prime} y\right)(\beta) z$, with $\beta:: p \wedge q \Rightarrow q \wedge p$ and $\gamma:: \mathbf{1} \wedge\left(r \wedge\left(q \wedge r^{\prime}\right)\right) \Rightarrow r \wedge(\mathbf{1} \wedge p)$.

v) $(\theta, \mathbf{1}) \| \nVdash \forall x \forall y(\mathcal{J}(x), y \sqsubseteq x \rightarrow \mathcal{J}(y))$

where $\theta=\lambda x \lambda y\left((\chi) \lambda z\left(\left(\left(\chi^{\prime}\right)\left(\bar{\alpha}_{0} y\right) \lambda z^{\prime}\left(\chi^{\prime} x\right)(\beta) z^{\prime}\right)(\alpha) z\right)(\gamma) z\right.$, with

$\alpha:: \mathbf{1} \wedge\left(p^{\prime} \wedge(r \wedge q)\right) \Rightarrow(r \wedge \mathbf{1}) \wedge(\mathbf{1} \wedge \mathbf{1}) ; \quad \alpha^{\prime}:: \mathbf{1} \wedge\left(p^{\prime} \wedge\left(q^{\prime} \wedge q\right)\right) \Rightarrow q \wedge p^{\prime} ; \beta:: p \wedge q \Rightarrow q \wedge p$.

Proof.

i) Let $(\xi, p) \| \leftarrow \mathcal{J}(\mathbf{1})$; we must show that $(\bar{\alpha}, \mathbf{1}) \star(\xi, p) \cdot(\pi, q) \in \mathbb{\Perp}$, that is to say:

$(\bar{\alpha} \star \xi \cdot \pi, \mathbf{1} \wedge(p \wedge q)) \in \mathbb{I}$. But, from proposition 2.8 , we have:

$(\bar{\alpha} \star \xi \cdot \pi, \mathbf{1} \wedge(p \wedge q)) \succ(\xi \star \pi, p \wedge \mathbf{1}) \equiv(\xi, p) \star(\pi, \mathbf{1})$.

Now, we have $(\xi, p) \star(\pi, \mathbf{1}) \in \mathbb{\Perp}$ by hypothesis on $(\xi, p)$.

ii)Let $(\eta, p) \| \models \neg[q]$ and $(\pi, q) \in\|\mathcal{J}(q)\|$. We must show that $(\theta, \mathbf{1}) \star(\eta, p) \cdot(\pi, q) \in \mathbb{\Perp}$, i.e. $(\theta \star \eta \cdot \pi, \mathbf{1} \wedge(p \wedge q)) \in \mathbb{\Perp}$. Thus, let $\tau \in \mathbf{C}[\mathbf{1} \wedge(p \wedge q)]$; we must show that $\theta \star \eta \bullet \pi^{\tau} \in \Perp$.

From proposition 3.4, we have $\chi^{\prime} \eta \Vdash \mathrm{C}[p \wedge(\mathbf{1} \wedge \mathbf{1})], \mathrm{C}[q] \rightarrow \perp$.

Now, we have $\beta \tau \in \mathbf{C}[p \wedge(\mathbf{1} \wedge \mathbf{1})]$ and $\alpha \tau \in \mathbf{C}[q]$, therefore $\chi^{\prime} \eta \star \beta \tau \cdot \alpha \tau \cdot \pi \in \Perp$ thus

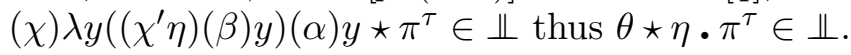

iii) Let $\left(\xi, p^{\prime}\right)\left\|-\mathcal{J}(p \wedge q), \quad\left(\eta, q^{\prime}\right)\right\| \Vdash \neg \mathcal{J}(p)$ and $(\pi, q) \in\|\mathcal{J}(q)\|$. We must show that:

$(\theta, \mathbf{1}) \star\left(\xi, p^{\prime}\right) \cdot\left(\eta, q^{\prime}\right) \cdot(\pi, q) \in \mathbb{\Perp}$, i.e. $\left(\theta \star \xi \cdot \eta \cdot \pi, \mathbf{1} \wedge\left(p^{\prime} \wedge\left(q^{\prime} \wedge q\right)\right)\right) \in \mathbb{\Perp}$.

From propositions 2.12(ii) and 2.8, it suffices to show:

$\left((\bar{\alpha})(\eta)(\bar{\beta}) \xi \star \pi, \mathbf{1} \wedge\left(p^{\prime} \wedge\left(q^{\prime} \wedge q\right)\right)\right) \in \mathbb{\Perp}$ then $\left(\eta \star \bar{\beta} \xi \cdot \pi, q^{\prime} \wedge\left(\left(q \wedge p^{\prime}\right) \wedge \mathbf{1}\right)\right) \in \mathbb{\Perp}$, that is:

$\left(\eta, q^{\prime}\right) \star\left(\bar{\beta} \xi, q \wedge p^{\prime}\right) \cdot(\pi, \mathbf{1}) \in \mathbb{H}$.

By hypothesis on $\left(\eta, q^{\prime}\right)$, we have now to show that $\left(\bar{\beta} \xi, q \wedge p^{\prime}\right) \| \mathcal{J}(p)$, i.e.:

$\left(\bar{\beta} \xi, q \wedge p^{\prime}\right) \star(\varpi, p) \in \mathbb{\Perp}$, or else $\left(\bar{\beta} \xi \star \varpi,\left(q \wedge p^{\prime}\right) \wedge p\right) \in \mathbb{\Perp}$ for all $\varpi \in \Pi$.

But, by proposition 2.8, we have:

$\left(\bar{\beta} \xi \star \varpi,\left(q \wedge p^{\prime}\right) \wedge p\right) \succ\left(\xi \star \varpi, p^{\prime} \wedge(p \wedge q)\right) \equiv\left(\xi, p^{\prime}\right) \star(\varpi, p \wedge q) \in \mathbb{\Perp}$ by hypothesis on $\left(\xi, p^{\prime}\right)$.

iv) Let $(\xi, q) \| \mathcal{J}(p)$ and $(\eta, r) \| \forall \forall q(\neg \mathrm{C}[p \wedge q] \rightarrow \mathcal{J}(q))$; we must show that:

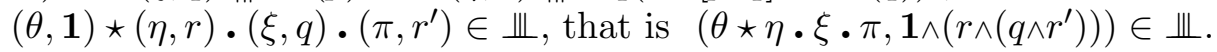

From proposition [3.4(i), we have $\chi^{\prime} \xi \Vdash \neg \mathbf{C}[q \wedge p]$. Let $\tau \in \mathbf{C}[p \wedge q]$, thus $\beta \tau \in \mathbf{C}[q \wedge p]$ therefore $\chi^{\prime} \xi \star \beta \tau, \rho \in \Perp$ for every $\rho \in \Pi$. Therefore, we have $\lambda x\left(\chi^{\prime} \xi\right)(\beta) x \star \tau \cdot \rho \in \Perp$, thus $\lambda z\left(\chi^{\prime} \xi\right)(\beta) z \Vdash \neg \mathrm{C}[p \wedge q]$. From proposition 3.4(iii), we have $\left(\lambda z\left(\chi^{\prime} \xi\right)(\beta) z, \mathbf{1}\right) \| \neg \mathrm{C}[p \wedge q]$.

By hypothesis on $(\eta, r)$, we thus have $(\eta, r) \star\left(\lambda z\left(\chi^{\prime} \xi\right)(\beta) z, \mathbf{1}\right) \cdot(\pi, q) \in \mathbb{\Perp}$, i.e.:

$\left(\eta \star \lambda z\left(\chi^{\prime} \xi\right)(\beta) z \cdot \pi, r \wedge(\mathbf{1} \wedge q)\right) \in \mathbb{\Perp}$, thus $\left((\bar{\gamma})(\eta) \lambda z\left(\chi^{\prime} \xi\right)(\beta) z \star \pi, \mathbf{1} \wedge\left(r \wedge\left(q \wedge r^{\prime}\right)\right)\right) \in \mathbb{\Perp}$ (proposition 2.8) and therefore $\left(\theta \star \eta \cdot \xi \bullet \pi, \mathbf{1} \wedge\left(r \wedge\left(q \wedge r^{\prime}\right)\right)\right) \in \mathbb{\Perp}$.

v) Let $\left(\xi, p^{\prime}\right) \| \vdash \mathcal{J}(p)$ and $(\eta, r) \| \leftarrow q \sqsubseteq p$; we must show that:

$(\theta, \mathbf{1}) \star\left(\xi, p^{\prime}\right) \cdot(\eta, r) \cdot(\pi, q) \in \mathbb{\Perp}$ for all $\pi \in \Pi$, that is $\left(\theta \star \xi \cdot \eta \cdot \pi, \mathbf{1} \wedge\left(p^{\prime} \wedge(r \wedge q)\right)\right) \in \mathbb{\Perp}$.

From proposition $3.4(\mathrm{i})$, we have $\chi^{\prime} \xi \nVdash \neg \mathrm{C}\left[p^{\prime} \wedge p\right]$, thus $\lambda z^{\prime}\left(\chi^{\prime} \xi\right)(\beta) z^{\prime} \Vdash \neg \mathrm{C}\left[p \wedge p^{\prime}\right]$ : indeed, if $\tau \in \mathrm{C}\left[p \wedge p^{\prime}\right]$ and $\rho \in \Pi$, we have $\lambda z^{\prime}\left(\chi^{\prime} \xi\right)(\beta) z^{\prime} \star \tau \cdot \rho \succ\left(\chi^{\prime} \xi\right)(\beta) \tau \star \rho \in \Perp$ since $\beta \tau \in \mathrm{C}\left[p^{\prime} \wedge p\right]$. Then, from proposition 3.4(iii), we have $\left(\lambda z^{\prime}\left(\chi^{\prime} \xi\right)(\beta) z^{\prime}, \mathbf{1}\right) \| \neg \neg\left[p \wedge p^{\prime}\right]$. But, by hypothesis on $(\eta, r)$, we have $(\eta, r) \| \vdash\left(\neg \mathrm{C}\left[p \wedge p^{\prime}\right] \rightarrow \neg \mathrm{C}\left[q \wedge p^{\prime}\right]\right)$. It follows that:

$(\eta, r)\left(\lambda z^{\prime}\left(\chi^{\prime} \xi\right)(\beta) z^{\prime}, \mathbf{1}\right) \| \neg-\mathcal{C}\left[q \wedge p^{\prime}\right]$, i.e. $\quad\left(\left(\bar{\alpha}_{0} \eta\right) \lambda z^{\prime}\left(\chi^{\prime} \xi\right)(\beta) z^{\prime}, r \wedge \mathbf{1}\right) \| \neg \mathbf{C}\left[q \wedge p^{\prime}\right]$.

From proposition 3.4(ii), we have $\left(\chi^{\prime}\right)\left(\bar{\alpha}_{0} \eta\right) \lambda z^{\prime}\left(\chi^{\prime} \xi\right)(\beta) z^{\prime} \Vdash \mathrm{C}[(r \wedge \mathbf{1}) \wedge(\mathbf{1} \wedge \mathbf{1})], \mathrm{C}\left[q \wedge p^{\prime}\right] \rightarrow \perp$. 
Let $\tau \in \mathbf{C}\left[\mathbf{1} \wedge\left(p^{\prime} \wedge(r \wedge q)\right)\right]$, therefore $\alpha \tau \in \mathbf{C}[(r \wedge \mathbf{1}) \wedge(\mathbf{1} \wedge \mathbf{1})]$ and $\alpha^{\prime} \tau \in \mathbf{C}\left[q \wedge p^{\prime}\right]$.

Thus, we have:

$\left(\left(\left(\chi^{\prime}\right)\left(\bar{\alpha}_{0} \eta\right) \lambda z^{\prime}\left(\chi^{\prime} \xi\right)(\beta) z^{\prime}\right)(\alpha) \tau\right)(\gamma) \tau \star \pi \in \Perp \Perp \Perp$, therefore:

$(\chi) \lambda z\left(\left(\left(\chi^{\prime}\right)\left(\bar{\alpha}_{0} \eta\right) \lambda z^{\prime}\left(\chi^{\prime} \xi\right)(\beta) z^{\prime}\right)(\alpha) z\right)\left(\alpha^{\prime}\right) z \star \pi^{\tau} \in \mathbb{\Perp}$. In other words:

$\left((\chi) \lambda z\left(\left(\left(\chi^{\prime}\right)\left(\bar{\alpha}_{0} \eta\right) \lambda z^{\prime}\left(\chi^{\prime} \xi\right)(\beta) z^{\prime}\right)(\alpha) z\right)\left(\alpha^{\prime}\right) z \star \pi, \mathbf{1} \wedge\left(p^{\prime} \wedge(r \wedge q)\right)\right) \in \mathbb{\Perp}$

or else, from proposition 2.12(ii): $\left(\theta \star \xi \bullet \eta \cdot \pi, \mathbf{1} \wedge\left(p^{\prime} \wedge(r \wedge q)\right)\right) \in \mathbb{\Perp}$.

Theorem 3.6 (Density).

For each function $\phi: P \rightarrow P$, we have:

$(\theta, \mathbf{1}) \| \Vdash \forall x(\neg \mathrm{C}[x \wedge \phi(x)] \rightarrow \mathcal{J}(x)), \forall x \mathcal{J}(x \wedge \phi(x)) \rightarrow \perp$

where $\theta=(\bar{\beta}) \lambda x \lambda y(x)(\vartheta) y, \quad \vartheta=(\chi) \lambda d \lambda x \lambda y\left(\chi^{\prime} x\right)(\alpha) y$;

with $\alpha:: q \wedge r \Rightarrow q \wedge(q \wedge r) ; \beta:: \mathbf{1} \wedge(p \wedge(q \wedge r)) \Rightarrow p \wedge(\mathbf{1} \wedge q)$.

Proof. Let $(\xi, p)\|\mapsto \forall x(\neg \mathrm{C}[x \wedge \phi(x)] \rightarrow \mathcal{J}(x)),(\eta, q)\| \vdash \forall x \mathcal{J}(x \wedge \phi(x))$ and $(\pi, r) \in \mathbf{\Pi}$.

we must show that $(\theta \star \xi \bullet \eta \bullet \pi, \mathbf{1} \wedge(p \wedge(q \wedge r))) \in \mathbb{\Perp}$; thus, let $\tau_{0} \in \mathbf{C}[\mathbf{1} \wedge(p \wedge(q \wedge r))]$. We must show $\theta \star \xi \cdot \eta \cdot \pi^{\tau_{0}} \in \mathbb{\Perp}$.

We first show that $(\vartheta \eta, \mathbf{1}) \| \vdash \neg[q \wedge \phi(q)]$.

Thus, let $\left(\varpi, r^{\prime}\right) \in \boldsymbol{\Pi}$ and $\tau \in \mathbf{C}[q \wedge \phi(q)]$; we must show $(\vartheta \eta, \mathbf{1}) \star(\tau, \mathbf{1}) \cdot\left(\varpi, r^{\prime}\right) \in \mathbb{\Perp}$ i.e. $\left(\vartheta \eta \star \tau \cdot \varpi, \mathbf{1} \wedge\left(\mathbf{1} \wedge r^{\prime}\right)\right) \in \mathbb{\Perp}$ or else $\vartheta \eta \star \tau \cdot \varpi^{\tau^{\prime}} \in \mathbb{\Perp}$ for each $\left.\tau^{\prime} \in \mathbf{C}\left[\mathbf{1} \wedge\left(\mathbf{1} \wedge r^{\prime}\right)\right]\right)$.

Now, $\vartheta \eta \star \tau \cdot \varpi^{\tau^{\prime}} \succ \eta \star \varpi^{\alpha \tau}$ and $\alpha \tau \in \mathrm{C}[q \wedge(q \wedge \phi(q))]$. Thus, it suffices to show:

$(\eta \star \varpi, q \wedge(q \wedge \phi(q))) \in \mathbb{\Perp}$ or else $(\eta, q) \star(\varpi, q \wedge \phi(q)) \in \mathbb{\Perp}$.

But this follows from the hypothesis on $(\eta, q)$, which implies $(\eta, q) \| \Vdash \mathcal{J}(q \wedge \phi(q))$.

By hypothesis on $\xi$, we have $(\xi, p) \| \vdash \neg[q \wedge \phi(q)] \rightarrow \mathcal{J}(q)$. It follows that:

$(\xi, p) \star(\vartheta \eta, \mathbf{1}) \cdot(\pi, q) \in \mathbb{\Perp}$, that is $(\xi \star \vartheta \eta \cdot \pi, p \wedge(\mathbf{1} \wedge q)) \in \mathbb{\Perp}$.

But we have $\left.\tau_{0} \in \mathrm{C}[\mathbf{1} \wedge(p \wedge(q \wedge r))]\right)$, thus $\beta \tau_{0} \in \mathrm{C}[p \wedge(\mathbf{1} \wedge q)]$. It follows that $\xi \star \vartheta \eta \bullet \pi^{\beta \tau_{0}} \in \Perp \Perp \Perp$.

This gives the desired result, since $\theta \star \xi \cdot \eta \cdot \pi^{\tau_{0}} \succ \xi \star \vartheta \eta \cdot \pi^{\beta \tau_{0}}$.

\section{Countable downward Chain COndition}

In this section, we consider a standard realizability algebra $\mathcal{A}$ and a $\mathcal{A}$-model $\mathcal{M}$. We suppose that the set $P$ (domain of variation of individual variables) has a power $\geq 2^{\aleph_{0}}$. We choose a surjection $\varepsilon: P \rightarrow \mathcal{P}(\Pi)^{\mathbb{N}}$ and we define a binary predicate in the model $\mathcal{M}$, which we denote also by $\varepsilon$, by putting:

$$
\|n \varepsilon p\|=\varepsilon(p)(n) \text { if } n \in \mathbb{N} ;\|n \varepsilon p\|=\emptyset \text { if } n \notin \mathbb{N}
$$

(we use, for the predicate $\varepsilon$, the notation $n \varepsilon p$ instead of $\varepsilon(n, p)$ ).

Therefore, the predicate $\varepsilon$ enables us to associate, with each individual, a set of integers which are its elements. Proposition 4.1 shows that the following axiom is realized:

For every set, there exists an individual which has the same integer elements.

This axiom will be called axiom of representation of predicates on $\mathbb{N}$ and denoted by RPN.

Proposition 4.1 (RPN).

$\lambda x(x) \underline{0} \underline{0} \Vdash \forall X \exists x \forall n^{e n t}(X n \leftrightarrow n \varepsilon x)$.

Proof. This formula is $\forall X(\forall x[\forall n(\operatorname{ent}(n), X n \rightarrow n \varepsilon x), \forall n(\operatorname{ent}(n), n \varepsilon x \rightarrow X n) \rightarrow \perp] \rightarrow \perp)$.

Thus, we consider a unary parameter $\mathcal{X}: P \rightarrow \mathcal{P}(\Pi)$ and a term $\xi \in \Lambda$ such that:

$\xi \Vdash \forall x[\forall n(\operatorname{ent}(n), \mathcal{X} n \rightarrow n \varepsilon x), \forall n(\operatorname{ent}(n), n \varepsilon x \rightarrow \mathcal{X} n) \rightarrow \perp]$.

We must show that $\lambda x(x) \underline{0} \underline{0} \star \xi \bullet \pi \in \mathbb{\Perp}$, or else $\xi \star \underline{0} \bullet \underline{0} \bullet \pi \in \Perp$ for every stack $\pi \in \Pi$.

By definition of $\varepsilon$, there exists $p_{0} \in P$ such that $\mathcal{X} n=\left\|n \varepsilon p_{0}\right\|$ for every integer $n$. 
But, we have: $\xi \Vdash \forall n\left(\operatorname{ent}(n), \mathcal{X} n \rightarrow n \varepsilon p_{0}\right), \forall n\left(\operatorname{ent}(n), n \varepsilon p_{0} \rightarrow \mathcal{X} n\right) \rightarrow \perp$.

Thus, it suffices to show that $\underline{0} \Vdash \forall n\left(\operatorname{ent}(n), \mathcal{X} n \rightarrow n \varepsilon p_{0}\right)$

and $\underline{0} \Vdash \forall n\left(\operatorname{ent}(n), n \varepsilon p_{0} \rightarrow \mathcal{X} n\right)$.

Recall that the predicate ent $(x)$ is defined as follows:

$|\operatorname{ent}(n)|=\{\underline{n}\} \quad$ if $n \in \mathbb{N}$ and $|\operatorname{ent}(n)|=\emptyset$ if $n \notin \mathbb{N}$.

Therefore, we have to show:

$\underline{0} \star \underline{n} \cdot \eta \cdot \rho \in \mathbb{\Perp}$ for all $n \in \mathbb{N}, \eta \nVdash \mathcal{X}(n)$ and $\rho \in\left\|n \varepsilon p_{0}\right\|$;

$\underline{0} \star \underline{n} \cdot \eta^{\prime} \cdot \rho^{\prime} \in \Perp$ for all $n \in \mathbb{N}, \eta^{\prime} \| n \varepsilon p_{0}$ and $\rho^{\prime} \in \mathcal{X}(n)$.

But this follows from $\eta \star \rho \in \Perp$ and $\eta^{\prime} \star \rho^{\prime} \in \Perp \Perp \Perp$, which is trivially true, since $\mathcal{X} n=\left\|n \varepsilon p_{0}\right\|$.

We suppose now that $\{\mathbf{C}, \wedge, \mathbf{1}\}$ is a forcing structure in $\mathcal{M}$. Then we define also the symbol $\varepsilon$ in the $\mathcal{B}$-model $\mathcal{N}$ by putting:

$\|n \varepsilon p\|=\|n \varepsilon p\| \times\{\mathbf{1}\}$ for $n, p \in P$. In other words

$\|n \varepsilon p\|=\{(\pi, \mathbf{1}) ; \pi \in \varepsilon(p)(n)\}$ if $n \in \mathbb{N} ;\|n \varepsilon p\|=\emptyset$ if $n \notin \mathbb{N}$.

Proposition 4.2. The predicate $\varepsilon^{+}(q, n, p)$ is $q=\mathbf{1} \mapsto n \varepsilon p$.

The formula $q \mathbb{t} \varepsilon p \quad$ is $\quad \mathrm{C}[q \wedge \mathbf{1}] \rightarrow n \varepsilon p$.

Proof. Immediate, by definition of $\|n \varepsilon p\| \|$.

\section{Proposition 4.3.}

i) $\xi \Vdash(\mathrm{C}[p] \rightarrow n \varepsilon q) \Rightarrow(\delta \xi, p) \| n n \varepsilon q$ where $\delta=\lambda x(\chi) \lambda y(x)(\alpha) y$ and $\alpha:: p \wedge \mathbf{1} \Rightarrow p$.

ii) $(\xi, p) \| n \varepsilon q \Rightarrow \delta^{\prime} \xi \Vdash(\mathrm{C}[p] \rightarrow n \varepsilon q)$ where $\delta^{\prime}=\lambda x \lambda y\left(\chi^{\prime} x\right)\left(\alpha^{\prime}\right) y$ and $\alpha^{\prime}:: p \Rightarrow p \wedge \mathbf{1}$.

Proof.

We have $(\xi, p) \| n \varepsilon p \Leftrightarrow(\xi, p) \star(\pi, \mathbf{1}) \in \mathbb{\Perp}$ for all $\pi \in\|n \varepsilon p\|$, or else:

$(\xi, p) \| n \varepsilon p \Leftrightarrow \xi \star \pi^{\tau} \in \Perp$ for each $\tau \in \mathrm{C}[p \wedge \mathbf{1}]$ and $\pi \in\|n \varepsilon p\|$.

i) Suppose that $\xi \Vdash(\mathrm{C}[p] \rightarrow n \varepsilon q), \tau \in \mathrm{C}[p \wedge \mathbf{1}]$ and $\pi \in\|n \varepsilon p\|$. Then,we have:

$\delta \xi \star \pi^{\tau} \succ \xi \star \alpha \tau \cdot \pi \in \Perp \Perp \Perp$, since $\alpha \tau \in \mathrm{C}[p]$.

ii) Suppose that $(\xi, p) \Vdash n \varepsilon q, \tau \in \mathrm{C}[p]$ and $\pi \in\|n \varepsilon p\|$. Then,we have:

$\delta^{\prime} \xi \star \tau \cdot \pi \succ \xi \star \pi^{\alpha^{\prime} \tau} \in \mathbb{\Perp}$, since $\alpha^{\prime} \tau \in \mathrm{C}[p \wedge \mathbf{1}]$.

The notion of first order formula has been defined previously (see theorem 3.3). We extend this definition with the following clause:

- $t \varepsilon u$ is first order, for all terms $t, u$.

Proposition 4.3 shows that theorem 3.3 remains true for this extended notion.

We say that the forcing structure $\{\mathbf{C}, \wedge, \mathbf{1}\}$ satisfies the countable downward chain condition (in abridged form c.d.c.) if there exists a proof-like term cdc such that:

$$
\begin{aligned}
\text { cdc } \Vdash & \forall X\left[\forall n^{\text {ent }} \exists p X(n, p), \forall n^{\text {ent }} \forall p \forall q(X(n, p), X(n, q) \rightarrow p=q),\right. \\
& \forall n^{\text {ent }} \forall p \forall q(X(n, p), X(s n, q) \rightarrow q \sqsubseteq p) \rightarrow \\
& \left.\exists p^{\prime}\left\{\forall n^{\text {ent }} \forall p\left(X(n, p) \rightarrow p^{\prime} \sqsubseteq p\right),\left(\forall n^{\text {ent }^{\prime}} \forall p(X(n, p) \rightarrow \mathrm{C}[p]) \rightarrow \mathrm{C}\left[p^{\prime}\right]\right)\right\}\right] .
\end{aligned}
$$

The intuitive meaning of this formula is:

If $X(n, p)$ is a decreasing sequence of conditions, then there exists a condition $p^{\prime}$ which is less than all of them ; moreover, if all these conditions are non trivial, then $p^{\prime}$ is non trivial.

We intend, in this section to prove the: 
Theorem 4.4 (Conservation of reals).

If the c.d.c. is verified, then there exists a proof-like term $\mathrm{crl}$ such that:

(crl, 1) $\| \Vdash \forall X \exists x \forall n^{e n t}(X n \leftrightarrow n \varepsilon x)$.

Proof. This means that the axiom RPN, which is realized in the $\mathcal{A}$-model $\mathcal{M}$ (see proposition 4.1) is also realized in the generic $\mathcal{B}$-model $\mathcal{N}$.

\section{Notation.}

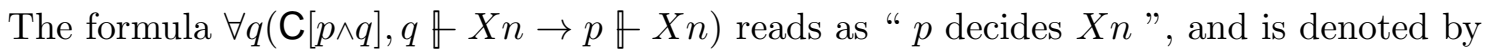

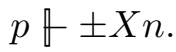

It can also be written as $\forall q \forall r\left(\mathrm{C}[p \wedge q], q \mathbb{R} X n, \mathrm{C}[p \wedge r] \rightarrow X^{+}(r, n)\right)$.

If $\mathcal{X}: P \rightarrow \mathcal{P}(\Pi \times P)$ is a unary predicate in the $\mathcal{B}$-model $\mathcal{N}$,

and $\mathcal{X}^{+}: P^{2} \rightarrow \mathcal{P}(\Pi)$ is the corresponding binary predicate in the standard $\mathcal{A}$-model $\mathcal{M}$,

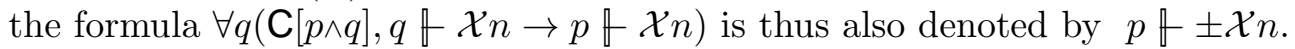

Theorem 4.5. If the c.d.c. is verified, there exists a proof-like term dec such that: dec $\Vdash \forall X \forall p_{0} \exists p^{\prime}\left\{\left(\mathbf{C}\left[p_{0}\right] \rightarrow \mathbf{C}\left[p^{\prime}\right]\right), p^{\prime} \sqsubseteq p_{0}, \forall n^{e n t}\left(p^{\prime} \mathbb{E} \pm X n\right)\right\}$.

Remark. This formula means that, for any predicate $X$, the set of conditions which decide $X n$ for all integers $n$ is dense.

We first show how theorem 4.4 can be deduced from this theorem 4.5 ,

From theorem 3.2, it is sufficient to find a proof-like term crl0 such that:

crlo $\Vdash \mathbf{1} \Vdash \forall X \exists x \forall n^{\mathrm{ent}}(X n \leftrightarrow n \varepsilon x)$

or else, since $\mathbf{1} \mathbb{A} \neg A \equiv p_{0}\left(\left(p_{0} \mathbb{t} A\right), \mathrm{C}\left[\mathbf{1} \wedge p_{0}\right] \rightarrow \perp\right)$ :

crl0 $\Vdash \forall X \forall p_{0}\left[\left(p_{0} \Vdash \forall q\left\{\forall n^{\mathrm{ent}}(X n \leftrightarrow n \varepsilon q) \rightarrow \perp\right\}\right), \mathrm{C}\left[\mathbf{1} \wedge p_{0}\right] \rightarrow \perp\right]$.

From theorem 4.5, it is sufficient to find a proof-like term crl1 such that:

crl1 $\Vdash \forall X \forall p_{0} \forall p^{\prime}\left\{\left(\mathbf{C}\left[p_{0}\right] \rightarrow \mathbf{C}\left[p^{\prime}\right]\right), p^{\prime} \sqsubseteq p_{0}, \forall n^{\text {ent }}\left(p^{\prime} \mathbb{E} \pm X n\right)\right.$,

$\left.\left(p_{0} \Vdash \forall q\left(\forall n^{\mathrm{ent}}(X n \leftrightarrow n \varepsilon q) \rightarrow \perp\right)\right), \mathrm{C}\left[\mathbf{1} \wedge p_{0}\right] \rightarrow \perp\right\}$.

It is sufficient to find a proof-like term crl2 such that:

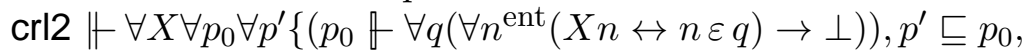
$\left.\forall n^{\text {ent }}\left(p^{\prime} \mathbb{R} \pm X n\right), \mathrm{C}\left[p^{\prime}\right] \rightarrow \perp\right\}$

Indeed, we take then $\mathrm{crl1}=\lambda x \lambda y \lambda z \lambda u \lambda v((x)(\operatorname{crl} 2) u y z)(\delta) v$ with $\delta:: \mathbf{1} \wedge p \Rightarrow p$;

(recall that the formula $\mathbf{C}\left[p_{0}\right] \rightarrow \mathbf{C}\left[p^{\prime}\right]$ is written, in fact, as $\neg \mathbf{C}\left[p^{\prime}\right] \rightarrow \neg \mathbf{C}\left[p_{0}\right]$ ).

We fix $\mathcal{X}^{+}: P^{2} \rightarrow \mathcal{P}(\Pi), p_{0}, p^{\prime} \in P, \xi \Vdash\left(p_{0} \mathbb{H} \forall q\left(\forall n^{\text {ent }}(\mathcal{X} n \leftrightarrow n \varepsilon q) \rightarrow \perp\right)\right), \eta \Vdash p^{\prime} \sqsubseteq p_{0}$,

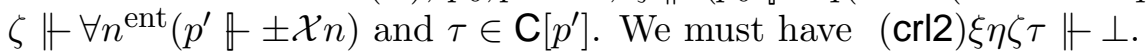

We choose $q_{0} \in P$ such that we have $\left\|n \varepsilon q_{0}\right\|=\left\|p^{\prime} \mathbb{E} \mathcal{X} n\right\|$ for all $n \in \mathbb{N}$, which is possible, by definition of $\varepsilon$.

We trivially have $\xi \Vdash\left(p_{0} \mathbb{t}\left(\forall n^{\mathrm{ent}}\left(n \varepsilon q_{0} \rightarrow \mathcal{X} n\right), \forall n^{\mathrm{ent}}\left(\mathcal{X}_{n} \rightarrow n \varepsilon q_{0}\right) \rightarrow \perp\right)\right)$.

But, the formula $p_{0} \mathbb{E}\left(\forall n^{\text {ent }}\left(n \varepsilon q_{0} \rightarrow \mathcal{X} n\right), \forall n^{\text {ent }}\left(\mathcal{X} n \rightarrow n \varepsilon q_{0}\right) \rightarrow \perp\right)$ is written as:

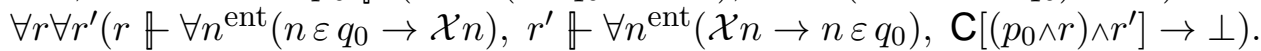

Replacing $r$ and $r^{\prime}$ with $p^{\prime}$, we obtain:

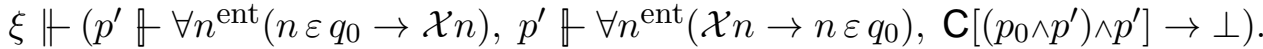

From $\tau \in \mathbf{C}\left[p^{\prime}\right]$ and $\eta \Vdash \forall r\left(\neg \mathbf{C}\left[p_{0} \wedge r\right] \rightarrow \neg \mathbf{C}\left[p^{\prime} \wedge r\right]\right)$, we deduce that:

$\lambda h((\eta) \lambda x(h)(\beta) x)(\alpha) \tau \Vdash \neg \neg \mathbf{C}\left[\left(p_{0} \wedge p^{\prime}\right) \wedge p^{\prime}\right]$

where $\alpha, \beta$ are $\mathrm{C}$-expressions such that $\alpha: p \Rightarrow p \wedge p ; \beta:: p \wedge q \Rightarrow(p \wedge q) \wedge q$.

Thus, we have:

(1) $\lambda y \lambda z((\eta) \lambda x(\xi y z)(\beta) x)(\alpha) \tau \Vdash$

$$
\left(p^{\prime} \mathbb{E} \forall n^{\mathrm{ent}}\left(n \varepsilon q_{0} \rightarrow \mathcal{X} n\right)\right),\left(p^{\prime} \mathbb{E} \forall n^{\mathrm{ent}}\left(\mathcal{X} n \rightarrow n \varepsilon q_{0}\right)\right) \rightarrow \perp .
$$




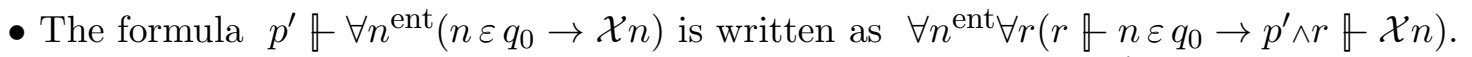

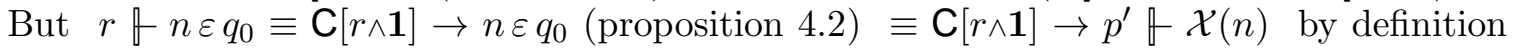

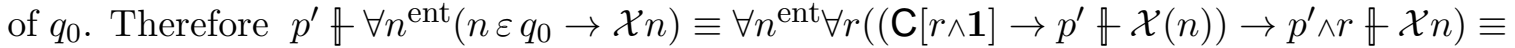
$\forall n^{\text {ent }} \forall r \forall q^{\prime}\left[\forall q\left(\mathrm{C}[r \wedge \mathbf{1}], \mathrm{C}\left[p^{\prime} \wedge q\right] \rightarrow \mathcal{X}^{+}(q, n)\right), \mathrm{C}\left[\left(p^{\prime} \wedge r\right) \wedge q^{\prime}\right] \rightarrow \mathcal{X}^{+}\left(q^{\prime}, n\right)\right]$.

Thus, we have:

(2) $\lambda d \lambda x \lambda y\left((x)\left(\alpha^{\prime}\right) y\right)\left(\beta^{\prime}\right) y \Vdash\left(p^{\prime} \mathbb{E} \forall n^{\text {ent }}\left(n \varepsilon q_{0} \rightarrow \mathcal{X} n\right)\right)$

with $\alpha^{\prime}::(p \wedge r) \wedge q \Rightarrow r \wedge \mathbf{1}$ and $\beta^{\prime}::(p \wedge r) \wedge q \Rightarrow p \wedge q$.

- The formula $p^{\prime} \mathbb{E} \forall n^{\text {ent }}\left(\mathcal{X} n \rightarrow n \varepsilon q_{0}\right)$ is written as $\forall n^{\text {ent }} \forall r\left(r \mathbb{X} n \rightarrow p^{\prime} \wedge r \mathbb{E} n \varepsilon q_{0}\right)$,

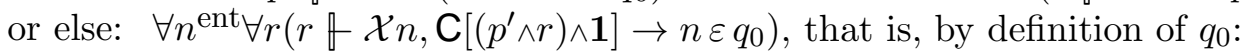

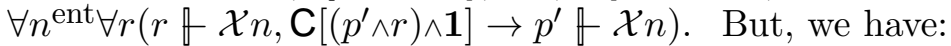

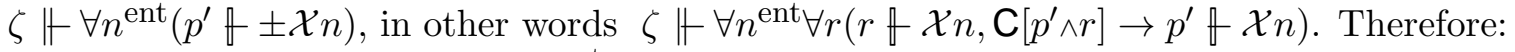
(3) $\lambda n \lambda x \lambda y(\zeta n x)\left(\alpha^{\prime \prime}\right) y \Vdash p^{\prime} \mathbb{H} \forall n^{\mathrm{ent}}\left(\mathcal{X} n \rightarrow n \varepsilon q_{0}\right)$ with $\alpha^{\prime \prime}::(p \wedge r) \wedge \mathbf{1} \Rightarrow p \wedge r$.

It follows from $(1,2,3)$ that:

$\left((\lambda y \lambda z((\eta) \lambda x(\xi y z)(\beta) x)(\alpha) \tau) \lambda d \lambda x \lambda y\left((x)\left(\alpha^{\prime}\right) y\right)\left(\beta^{\prime}\right) y\right) \lambda n \lambda x \lambda y(\zeta n x)\left(\alpha^{\prime \prime}\right) y \Vdash \perp$.

Therefore, we can put $\mathrm{crl} 2=$

$\lambda x_{0} \lambda y_{0} \lambda z_{0} \lambda u\left(\left(\lambda y \lambda z\left(\left(y_{0}\right) \lambda x\left(x_{0} y z\right)(\beta) x\right)(\alpha) u\right) \lambda d \lambda x \lambda y\left((x)\left(\alpha^{\prime}\right) y\right)\left(\beta^{\prime}\right) y\right) \lambda n \lambda x \lambda y\left(z_{0} n x\right)\left(\alpha^{\prime \prime}\right) y$.

The remaining of this section is devoted to the proof of theorem 4.5 .

Definition of a sequence by dependent choices. In this section, we are given a fixed element $p_{0} \in P$ and a finite sequence of formulas with parameters $\vec{F}\left(n, p, p^{\prime}\right)$. We are also given a proof-like term dse such that:

dse $\Vdash \forall n \forall p \exists p^{\prime} \vec{F}\left(n, p, p^{\prime}\right)$.

Remark. The aim of this section is to write down a formula $\Phi(x, y)$ which represents the graph of a function $\phi: \mathbb{N} \rightarrow P$ such that the formulas $\phi(0)=p_{0}$ and $\forall n^{\text {ent }} \vec{F}(n, \phi(n), \phi(n+1))$ are realized by proof-like terms. We shall only apply the results of this section to a particular sequence $\vec{F}$ of length 3.

From theorem 2.1(i) (axiom of choice for individuals), there exists a function $f: P^{3} \rightarrow P$ such that: $\quad \varsigma \| \forall n \forall p\left(\forall k^{\mathrm{ent}}(\vec{F}(n, p, f(n, p, k)) \rightarrow \perp) \rightarrow \forall p^{\prime}\left(\vec{F}\left(n, p, p^{\prime}\right) \rightarrow \perp\right)\right)$.

It follows that $\lambda x$ (dse) $(\varsigma) x \Vdash \forall n \forall p\left(\forall k^{\text {ent }}(\vec{F}(n, p, f(n, p, k)) \rightarrow \perp) \rightarrow \perp\right)$.

We define a function denoted by $\left(m_{\mathrm{i}} n\right)$, from $P^{2}$ into $P$, by putting, for $m, n \in P$ :

$\left(m_{\mathfrak{i}} n\right)=1$ if $m, n \in \mathbb{N}$ and $m<n ;\left(m_{\mathfrak{i}} n\right)=0$ otherwise.

Obviously, the relation $\left(m_{\mathfrak{j}} n\right)=1$ is well founded on $P$.

Thus, from theorem 1.11(ii), we have:

$\mathrm{Y} \Vdash \forall k\left(\forall l\left(\operatorname{ent}(l), \vec{F}(n, p, f(n, p, l)) \rightarrow\left(l_{i} k\right) \neq 1\right), \operatorname{ent}(k), \vec{F}(n, p, f(n, p, k)) \rightarrow \perp\right)$

$$
\rightarrow \forall k(\operatorname{ent}(k), \vec{F}(n, p, f(n, p, k)) \rightarrow \perp) \text {. }
$$

Therefore, if we set $\mathrm{Y}^{\prime}=\lambda x(\mathrm{Y}) \lambda y \lambda z(x) z y$, we have:

$$
\begin{aligned}
\mathrm{Y}^{\prime} \Vdash \forall k^{\mathrm{ent}}\left\{\forall l^{\mathrm{ent}}\left(\vec{F}[n, p, f(n, p, l)] \rightarrow\left(l_{\mathrm{i}} k\right) \neq 1\right), \vec{F}[n, p, f(n, p, k)] \rightarrow \underset{\perp}{\rightarrow}\right\} & \rightarrow \forall k^{\mathrm{ent}}(\vec{F}[n, p, f(n, p, k)] \rightarrow \perp) .
\end{aligned}
$$

Thus, we have:

$\lambda x($ dse $)(\varsigma)\left(\mathrm{Y}^{\prime}\right) x \Vdash \forall k^{\text {ent }}\left\{\forall l^{\text {ent }}\left(\vec{F}[n, p, f(n, p, l)] \rightarrow\left(l_{i} k\right) \neq 1\right), \vec{F}[n, p, f(n, p, k)] \rightarrow \perp\right\} \rightarrow \perp$. We define the formula $G(n, p, k) \equiv \forall l^{\text {ent }}\left(\vec{F}(n, p, f(n, p, l)) \rightarrow\left(l_{i} k\right) \neq 1\right)$ and the finite sequence of formulas $\vec{H}(n, p, k) \equiv\{G(n, p, k), \vec{F}(n, p, f(n, p, k))\}$. Then, we have shown: 
Lemma 4.6. dse $0 \Vdash \forall n \forall p \exists k^{e n t}\{\vec{H}(n, p, k)\}$, with dse $0=\lambda x$ (dse $)(\varsigma)\left(\mathrm{Y}^{\prime}\right) x$.

Remark. The meaning of $\vec{H}(n, p, k)$ is " $k$ is the least integer such that $\vec{F}(n, p, f(n, p, k))$ ".

Lemma 4.7. Let $c p$ be a proof-like term such that, for every $m, n \in \mathbb{N}$, we have:

$c p \star \underline{m} \cdot \underline{n} \cdot \xi \cdot \eta \cdot \zeta \cdot \pi \succ \xi \star \pi$ (resp. $\eta \star \pi, \zeta \star \pi)$ if $m<n$ (resp. $n<m, m=n$ ). Then:

i) $c p \| \forall m^{e n t} \forall n^{e n t}((m ; n) \neq 1,(n ; m) \neq 1, m \neq n \rightarrow \perp)$.

ii) dse1 $\Vdash \forall n \forall p \forall k^{e n t} \forall k^{\prime}$ ent $\left(\vec{H}(n, p, k), \vec{H}\left(n, p, k^{\prime}\right), k \neq k^{\prime} \rightarrow \perp\right)$

with dse $1=\lambda k \lambda k^{\prime} \lambda x \lambda \vec{y} \lambda x^{\prime} \lambda \vec{y}^{\prime}\left(\left(c p k^{\prime} k\right)(x) k^{\prime} \vec{y}^{\prime}\right)\left(x^{\prime}\right) k \vec{y}$, where $\vec{y}, \vec{y}^{\prime}$ are two sequences of distinct variables of the same length as the sequence $\vec{F}$.

Proof.

i) Trivial.

ii) Let $\xi \Vdash G(n, p, k), \vec{\eta} \Vdash \vec{F}(n, p, f(n, p, k)), \quad \xi^{\prime} \Vdash G\left(n, p, k^{\prime}\right), \vec{\eta}^{\prime} \Vdash \vec{F}\left(n, p, f\left(n, p, k^{\prime}\right)\right)$ and $\zeta \Vdash k \neq k^{\prime}$. We must show $\mathrm{cp} \star \underline{k}^{\prime} \cdot \underline{k} \cdot(\xi) \underline{k^{\prime}} \underline{\eta}^{\prime} \cdot\left(\xi^{\prime}\right) \underline{k} \vec{\eta} \cdot \zeta \cdot \pi \in \Perp$.

If $k=k^{\prime}$, it remains to prove $\zeta \star \pi \in \mathbb{\Perp}$; but this is true because we then have $\zeta \Vdash \perp$. If $k^{\prime}<k$, it remains to prove $\xi \star \underline{k}^{\prime} \cdot \vec{\eta}^{\prime} \cdot \pi \in \Perp \Perp$. This results immediately from:

$\xi \Vdash \forall k^{\prime}{ }^{\text {ent }}\left(\vec{F}\left(n, p, f\left(n, p, k^{\prime}\right)\right) \rightarrow\left(\bar{k}^{\prime} ; k\right) \neq 1\right)$ and thus:

$\xi \Vdash \operatorname{ent}\left(k^{\prime}\right), \vec{F}\left(n, p, f\left(n, p, k^{\prime}\right)\right) \rightarrow \perp$, since $k^{\prime}<k$.

We now define the binary predicate:

$\Phi(x, y) \equiv \forall X\left(\forall n \forall p \forall k^{\mathrm{ent}}(\vec{H}(n, p, k), X(n, p) \rightarrow X(s n, f(n, p, k))), X\left(0, p_{0}\right) \rightarrow X(x, y)\right)$

and we show that $\Phi(x, y)$ is a sequence of conditions (functional relation on $\mathbb{N}$ ) and also some other properties of $\Phi$.

Remark. Intuitively, the predicate $\Phi$ is the graph of the function $\phi$ of domain $\mathbb{N}$, recursively defined by the conditions: $\phi(0)=p_{0} ; \phi(n+1)=f^{\prime}(n, \phi(n))$

where $f^{\prime}(n, p)$ is $f(n, p, k)$ for the least $k$ such that $F(n, p, f(n, p, k))$. Unfortunately, we cannot introduce $f^{\prime}$ as a function symbol because, unlike $f$, it is not defined in the ground model.

\section{Lemma 4.8.}

i) $\lambda x \lambda y y \Vdash \Phi\left(0, p_{0}\right)$.

ii) $\lambda x(x) I I \Vdash \forall y\left(\Phi(0, y) \rightarrow y=p_{0}\right)$.

iii) rec $\Vdash \forall x \forall y \forall k^{e n t}(\vec{H}(x, y, k), \Phi(x, y) \rightarrow \Phi(s x, f(x, y, k)))$

where rec $=\lambda k \lambda x \lambda \vec{y} \lambda x^{\prime} \lambda z \lambda u(z k x \vec{y})\left(x^{\prime}\right) z u$

and $\vec{y}$ is a sequence of distinct variables of the same length as $\vec{F}$.

Proof.

i) Trivial.

ii) We define the binary predicate $\mathcal{X}: P^{2} \rightarrow \mathcal{P}(\Pi)$ by putting:

$\mathcal{X}(0, q)=\left\|q=p_{0}\right\|$ and $\mathcal{X}(p, q)=\emptyset$ for $p \neq 0$.

We replace $X$ with $\mathcal{X}$ in the definition of $\Phi(0, y)$. Since we have $s n \neq 0$ for all $n \in P$, we obtain $\|\Phi(0, y)\| \supset\left\|\top, p_{0}=p_{0} \rightarrow y=p_{0}\right\|$; hence the result.

iii) Let $\xi \Vdash G(x, y, k), \vec{\eta} \Vdash \vec{F}(x, y, f(x, y, k)), \quad \xi^{\prime} \Vdash \Phi(x, y)$,

$\zeta \Vdash \forall n \forall p \forall k^{\mathrm{ent}}(\vec{H}(n, p, k), X(n, p) \rightarrow X(s n, f(n, p, k)))$,

$v \| X\left(0, p_{0}\right)$ and $\pi \in\|X(s x, f(x, y, k))\|$.

Then $\xi^{\prime} \zeta v \Vdash X(x, y)$, therefore $\zeta \star \underline{k} \cdot \xi \cdot \vec{\eta} \cdot \xi^{\prime} \zeta v \cdot \pi \in \Perp$ i.e. $($ rec $) \underline{k} \xi \vec{\eta} \xi^{\prime} \zeta v \star \pi \in \Perp \Perp \Perp$. 
Lemma 4.9. cdc $1 \Vdash \forall n^{e n t} \exists p \Phi(n, p)$ where:

$\mathrm{cdc} 1=\lambda n((n) \lambda x \lambda y(x) \lambda z(\mathrm{~cd} 1) z y) \lambda x(x) \lambda x \lambda y y$

with $\mathrm{cd} 1=\lambda x \lambda y(\mathrm{dse} 0) \lambda l \lambda \vec{z}(y)(\mathrm{rec}) l \vec{z} x$;

$\vec{z}$ is a sequence of distinct variables of the same length as $\vec{H}$.

Proof by recurrence on $n$; we have $\lambda x \lambda y y \nVdash \Phi\left(0, p_{0}\right)$, therefore $\lambda x(x) \lambda x \lambda y y \Vdash \exists y \Phi(0, y)$.

We now show that $\mathrm{cd} 1 \nVdash \Phi(x, y) \rightarrow \exists y \Phi(s x, y)$.

Thus, we consider $\xi \Vdash \Phi(x, y), \eta \nVdash \forall y(\Phi(s x, y) \rightarrow \perp)$.

We have rec $\| \forall l^{\text {ent }}(\vec{H}(x, y, l), \Phi(x, y) \rightarrow \Phi(s x, f(x, y, l)))$ (lemma 4.8 ii),

$\eta \Vdash(\Phi(s x, f(x, y, l)) \rightarrow \perp)$, and therefore:

$\lambda l \lambda \vec{z}(\eta)$ (rec) $l \vec{z} \xi \Vdash \forall l^{\text {ent }}(\vec{H}(x, y, l) \rightarrow \perp)$, where $\vec{z}$ has the same length as $\vec{H}$.

Now, we have dse $\nVdash \exists k^{\text {ent }}\{\vec{H}(x, y, k)\}$ (lemma 4.6) ; therefore:

(dse 0$) \lambda l \lambda \vec{z}(\eta)($ rec $) l \vec{z} \xi \nVdash \perp$, that is (cd1) $\xi \eta \Vdash \perp$.

Thus, we have shown that $\operatorname{cd} 1 \Vdash \forall y(\Phi(x, y) \rightarrow \exists y \Phi(s x, y))$, and it follows that:

$\lambda x \lambda y(x) \lambda z(\mathrm{~cd} 1) z y \Vdash \exists y \Phi(x, y) \rightarrow \exists y \Phi(s x, y)$.

Lemma 4.10. There exists a proof-like term cdc2 such that:

cdc2 $\Vdash \forall n^{e n t} \forall p \forall q(\Phi(n, p), \Phi(n, q) \rightarrow p=q)$.

Proof. We give a detailed proof, by recurrence on $n$. It enables us to write explicitly the proof-like term cdc2.

For $n=0$, the lemma 4.8(ii) gives the result: $\Phi(0, p), \Phi(0, q) \rightarrow p=q$.

Let us fix $m$ and suppose that $\forall p \forall q(\Phi(m, p), \Phi(m, q) \rightarrow p=q)$.

We define the binary predicate:

$\Psi(n, q) \equiv \forall p \forall k^{\mathrm{ent}}(n=s m, \vec{H}(m, p, k), \Phi(m, p) \rightarrow q=f(m, p, k))$.

We show that $\Vdash \forall p \forall k^{\mathrm{ent}}(\vec{H}(n, p, k), \Phi(n, p) \rightarrow \Psi(s n, f(n, p, k)))$, that is to say: $\Vdash \forall p \forall q \forall k^{\text {ent }} \forall l^{\text {ent }}$

$$
\{\vec{H}(n, p, k), \Phi(n, p), s n=s m, \vec{H}(m, q, l), \Phi(m, q) \rightarrow f(n, p, k)=f(m, q, l)\} .
$$

But we have $\|s n=s m\|=\|n=m\|, \Phi(m, p), \Phi(m, q) \rightarrow p=q$ by hypothesis of recurrence ; $\vec{H}(m, p, k), \vec{H}(m, p, l) \rightarrow k=l$ (lemma 4.7(ii)), and it follows that $f(n, p, k)=f(m, q, l)$.

If we put $\Psi^{\prime}(x, y) \equiv \Phi(x, y) \wedge \Psi(x, y)$, we have:

$\nVdash \forall p \forall k^{\mathrm{ent}}\left(\vec{H}(n, p, k), \Psi^{\prime}(n, p) \rightarrow \Psi^{\prime}(s n, f(n, p, k))\right)$; we have also $\mathbb{H} \Psi^{\prime}\left(0, p_{0}\right)$. This shows that $\Vdash\left(\Phi(x, y) \rightarrow \Psi^{\prime}(x, y)\right)$ by making $X \equiv \Psi^{\prime}$ in the definition of $\Phi$.

Thus, we have $\Vdash \Phi(s m, q) \rightarrow \forall p \forall k^{\mathrm{ent}}(\vec{H}(m, p, k), \Phi(m, p) \rightarrow q=f(m, p, k))$.

It follows that:

$\Vdash \Phi(s m, q), \Phi\left(s m, q^{\prime}\right) \rightarrow$

$$
\forall p \forall k^{\mathrm{ent}}\left(\vec{H}(m, p, k), \Phi(m, p) \rightarrow(q=f(m, p, k)) \wedge\left(q^{\prime}=f(m, p, k)\right)\right)
$$

and therefore $\Vdash \Phi(s m, q), \Phi\left(s m, q^{\prime}\right) \rightarrow \forall p \forall k^{\mathrm{ent}}\left(\vec{H}(m, p, k), \Phi(m, p) \rightarrow q=q^{\prime}\right)$.

Thus, we obtain $\Vdash \Phi(s m, q), \Phi\left(s m, q^{\prime}\right) \rightarrow q=q^{\prime}$, since we have cdc1 $\Vdash \exists p \Phi(m, p)$ by lemma 4.9 and dse $0 \Vdash \forall p \exists k^{\text {ent }}\{\vec{H}(m, p, k)\}$ by lemma 4.6.

Resumption of the proof of theorem 4.5. In order to show theorem 4.5, we fix $p_{0} \in P$ and a binary predicate $\mathcal{X}: P^{2} \rightarrow \mathcal{P}(\Pi)$.

We have to find a proof-like term dec such that:

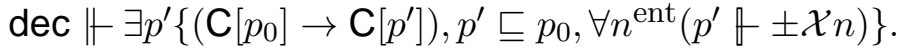


We apply the above results, taking for $\vec{F}\left(n, p, p^{\prime}\right)$ the sequence of three formulas: $\left\{\left(\mathrm{C}[p] \rightarrow \mathrm{C}\left[p^{\prime}\right]\right),\left(p^{\prime} \sqsubseteq p\right), p^{\prime} \mathbb{E} \pm \mathcal{X} n\right\}$.

Lemma 4.11 below gives a proof-like term dse such that dse $\Vdash \forall n \forall p \exists p^{\prime}\left\{\vec{F}\left(n, p, p^{\prime}\right)\right\}$.

Lemma 4.11. dse $\Vdash \forall p \exists p^{\prime}\left\{\vec{F}\left(n, p, p^{\prime}\right)\right\}$

where dse $=\lambda a(\lambda h(a I I) \lambda x \lambda y h) \lambda z(\mathrm{cc}) \lambda k\left((a \lambda x x z) \beta^{\prime}\right) \lambda x \lambda y(k)(y)(\alpha) x$

with $\beta^{\prime}=\lambda x \lambda y(x)(\beta) y, \quad \alpha::(p \wedge q) \wedge r \Rightarrow r \wedge q \quad$ and $\beta::(p \wedge q) \wedge r \Rightarrow p \wedge r$.

Proof. The formula we consider is written as $\forall p^{\prime}\left[\left(\mathrm{C}[p] \rightarrow \mathrm{C}\left[p^{\prime}\right]\right), p^{\prime} \sqsubseteq p,\left(p^{\prime} \mathbb{E} \pm \mathcal{X} n\right) \rightarrow\right.$ $\perp] \rightarrow \perp$.

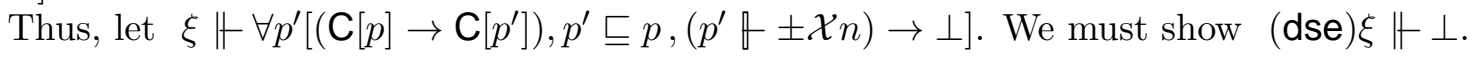

- We show that $\lambda h(\xi I I) \lambda x \lambda y h \Vdash \neg(p \Vdash \mathcal{X} n)$ :

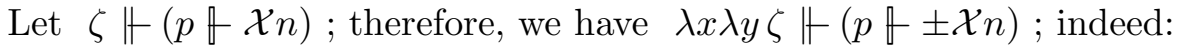

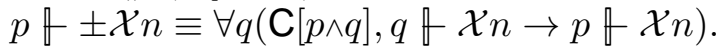

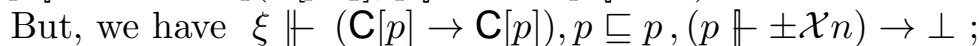

we have $I \Vdash \mathrm{C}[p] \rightarrow \mathrm{C}[p]$ and $I \Vdash p \sqsubseteq p \quad\left(\right.$ since $p^{\prime} \sqsubseteq p \equiv \forall q\left(\neg \mathrm{C}[p \wedge q] \rightarrow \neg \mathrm{C}\left[p^{\prime} \wedge q\right]\right)$ ).

Thus $(\xi I I) \lambda x \lambda y \zeta \Vdash \perp$, hence the result.

- We now show $\lambda z(\mathrm{cc}) \lambda k\left((\xi \lambda x x z) \beta^{\prime}\right) \lambda x \lambda y(k)(y)(\alpha) x \Vdash(p \Vdash \mathcal{X} n)$.

Thus, let $\tau \in \mathrm{C}[p \wedge q]$ and $\pi \in \mathcal{X}^{+}(q, n)$. We must show:

$\left((\xi \lambda x x \tau) \beta^{\prime}\right) \lambda x \lambda y\left(\mathrm{k}_{\pi}\right)(y)(\alpha) x \star \pi \in \Perp \Perp$. But, we have $\lambda x x \tau \Vdash \neg \neg \mathbf{C}[p \wedge q]$,

$\beta^{\prime} \Vdash p \wedge q \sqsubseteq p$ (lemma 4.12) and $\xi \Vdash(\neg \mathrm{C}[p \wedge q] \rightarrow \neg \mathrm{C}[p]), p \wedge q \sqsubseteq p,(p \wedge q \mathbb{\mathbb { X }} \pm \mathcal{X} n) \rightarrow \perp$; thus:

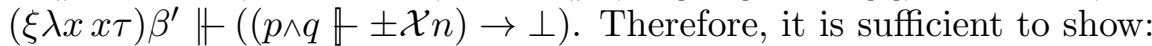

$\lambda x \lambda y\left(\mathrm{k}_{\pi}\right)(y)(\alpha) x \Vdash(p \wedge q \mathbb{X} \pm \mathcal{X} n)$, i.e.:

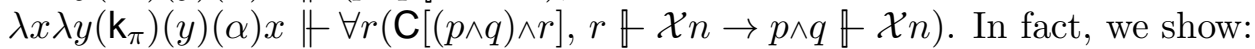

$\lambda x \lambda y\left(\mathrm{k}_{\pi}\right)(y)(\alpha) x \Vdash \forall r(\mathrm{C}[(p \wedge q) \wedge r], r \mathbb{X} n \rightarrow \perp)$.

Thus, let $v \in \mathrm{C}[(p \wedge q) \wedge r]$ and $\eta \Vdash(r \Vdash \mathcal{X} n)$. We must show:

$\left(\mathrm{k}_{\pi}\right)(\eta)(\alpha) v \star \rho \in \Perp$ for all $\rho \in \Pi$, i.e. $(\eta)(\alpha) v \star \pi \in \Perp \Perp$. But, we have $(\alpha) v \in \mathbf{C}[r \wedge q]$,

therefore $(\eta)(\alpha) v \Vdash \mathcal{X}^{+}(q, n)$, hence the result, since $\pi \in \mathcal{X}^{+}(q, n)$.

- It follows that $(\lambda h(\xi I I) \lambda x \lambda y h) \lambda z(\mathrm{cc}) \lambda k\left((\xi \lambda x x z) \beta^{\prime}\right) \lambda x \lambda y(k)(y)(\alpha) x \Vdash \perp$

i.e. (dse) $\xi \Vdash \perp$, which completes the proof.

Lemma 4.12. Let $\beta::(p \wedge q) \wedge r \Rightarrow p \wedge r$. Then $\lambda x \lambda y(x)(\beta) y \nVdash \forall p \forall q((p \wedge q) \sqsubseteq p)$.

Proof. This formula is written $\forall p \forall q \forall r(\neg \mathrm{C}[p \wedge r], \mathrm{C}[(p \wedge q) \wedge r] \rightarrow \perp)$.

Therefore, let $\xi \Vdash \neg \mathbf{C}[p \wedge r], \tau \in \mathbf{C}[(p \wedge q) \wedge r]$, thus $\beta \tau \in \mathbf{C}[p \wedge r]$ and $(\xi)(\beta) \tau \Vdash \perp$.

Thus, we obtain $\lambda x \lambda y(x)(\beta) y \star \xi \cdot \tau \cdot \pi \in \Perp$ for every $\pi \in \Pi$.

We propose now to apply the countable downward chain condition to the binary predicate $\Phi(x, y)$. Lemmas 4.9 and 4.10 show that the first two hypothesis of the c.d.c. are realized by cdc1 and cdc2. The third one is given by lemma 4.13 below.

Lemma 4.13. There exist two proof-like terms cdc3 and for such that:

i) cdc3 $\Vdash \forall n^{e n t} \forall p \forall q(\Phi(n, p), \Phi(s n, q) \rightarrow q \sqsubseteq p)$.

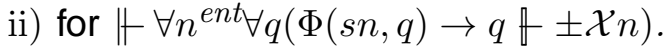

Proof. By lemma 4.8(iii), we have:

rec $\| \forall k^{\text {ent }}(\vec{H}(n, p, k), \Phi(n, p) \rightarrow \Phi(s n, f(n, p, k)))$. Using cdc2 (lemma 4.10), we get:

$\Vdash \forall k^{\mathrm{ent}}(\vec{H}(n, p, k), \Phi(n, p), \Phi(s n, q) \rightarrow q=f(n, p, k))$.

Now, $\vec{H}(n, p, k)$ is a sequence of four formulas, the last two of which are:

$f(n, p, k) \sqsubseteq p$ and $f(n, p, k) \mathbb{X} \mathcal{X}_{n}$. 
i) It follows first that $\nVdash \forall k^{\mathrm{ent}}(\vec{H}(n, p, k), \Phi(n, p), \Phi(s n, q) \rightarrow q \sqsubseteq p)$.

Hence the result, since we have dse $0 \Vdash \exists k^{\mathrm{ent}}\{\vec{H}(n, p, k)\}$ (lemma 4.6).

ii) It follows also that $\Vdash \forall k^{\text {ent }}(\vec{H}(n, p, k), \Phi(n, p), \Phi(s n, q) \rightarrow q \sharp \pm \mathcal{X} n)$.

Thus, we obtain $\Vdash \forall n^{\text {ent }} \forall q(\Phi(s n, q) \rightarrow q \mathbb{E} \pm \mathcal{X} n)$ since we have cdc1 $\Vdash \forall n^{\text {ent }} \exists p \Phi(n, p)$ (lemma 4.9) and dse $\Vdash \forall n \forall p \exists k^{\operatorname{ent}}\{\vec{H}(n, p, k)\}$ (lemma 4.6).

We can now apply the c.d.c. to the predicate $\Phi(x, y)$, which gives a proof-like term cdc0 such that cdc0 $\Vdash \exists p^{\prime}\left\{\vec{\Omega}\left(n, p, p^{\prime}\right)\right\}$ with :

$\vec{\Omega}\left(n, p, p^{\prime}\right) \equiv\left\{\forall n^{\text {ent }} \forall p\left(\Phi(n, p) \rightarrow p^{\prime} \sqsubseteq p\right), \forall n^{\text {ent }} \forall p(\Phi(n, p), \neg \mathbf{C}[p] \rightarrow \perp), \neg \mathbf{C}\left[p^{\prime}\right] \rightarrow \perp\right\}$.

Therefore, in order to complete the proof of theorem 4.5, it is sufficient to find proof-like terms dec0,dec1,dec2 such that:

$\operatorname{dec} 0 \Vdash \forall p^{\prime}\left(\vec{\Omega}\left(n, p, p^{\prime}\right), \neg \mathrm{C}\left[p_{0}\right], \mathrm{C}\left[p^{\prime}\right] \rightarrow \perp\right) ;$

$\operatorname{dec} 1 \Vdash \forall p^{\prime}\left(\vec{\Omega}\left(n, p, p^{\prime}\right) \rightarrow p^{\prime} \sqsubseteq p_{0}\right)$;

$\operatorname{dec} 2 \Vdash \forall p^{\prime}\left(\vec{\Omega}\left(n, p, p^{\prime}\right) \rightarrow \forall n^{\mathrm{ent}}\left(p^{\prime} \mathbb{E} \pm \mathcal{X} n\right)\right)$.

Thus, let $\omega_{0}, \omega_{1} \in \Lambda$ be such that:

$\omega_{0} \Vdash \forall n^{\mathrm{ent}} \forall p\left(\Phi(n, p) \rightarrow p^{\prime} \sqsubseteq p\right)$ and $\omega_{1} \Vdash \forall n^{\mathrm{ent}} \forall p(\Phi(n, p), \neg \mathbf{C}[p] \rightarrow \perp), \neg \mathbf{C}\left[p^{\prime}\right] \rightarrow \perp$

Applying lemma 4.8(i) with $n=0, p=p_{0}$, we obtain $\left(\omega_{0}\right) \lambda x \lambda y y \nVdash p^{\prime} \sqsubseteq p_{0}$.

Therefore, we can take $\operatorname{dec} 1=\lambda a \lambda b(a) \lambda x \lambda y y$.

Lemma 4.14. cdc4 $\Vdash\left(\mathrm{C}\left[p_{0}\right] \rightarrow \forall n^{e n t} \forall p(\Phi(n, p), \neg \mathrm{C}[p] \rightarrow \perp)\right)$

where cdc4 $=\lambda a \lambda b \lambda c\left(\left(b \lambda x_{0} \lambda x_{1} \lambda x_{2} \lambda x_{3} \lambda x \lambda y(x)\left(x_{1}\right) y\right) \lambda x x a\right) c$.

Proof. Let $\tau \in \mathrm{C}\left[p_{0}\right], \xi \nVdash \Phi(n, p)$ and $\eta \Vdash \neg \mathrm{C}[p]$.

Making $X(x, y) \equiv \neg \neg \mathrm{C}[y]$ in the definition de $\Phi$, we get:

$\xi \Vdash \forall n^{\prime} \forall p^{\prime} \forall k^{\text {ent }}\left(G\left[n^{\prime}, p^{\prime}, k\right], \vec{F}\left[n^{\prime}, p^{\prime}, f\left(n^{\prime}, p^{\prime}, k\right)\right], \neg \neg \mathrm{C}\left[p^{\prime}\right] \rightarrow \neg \neg \mathrm{C}\left[f\left(n^{\prime}, p^{\prime}, k\right)\right]\right)$,

We have $\lambda x(x) \tau \Vdash \neg \neg \mathbf{C}\left[p_{0}\right]$.

$$
\neg \neg \mathbf{C}\left[p_{0}\right], \neg \mathbf{C}[p] \rightarrow \perp \text {. }
$$

Moreover, since $\vec{F}\left[n^{\prime}, p^{\prime}, q\right] \equiv\left\{\left(\neg \mathrm{C}[q] \rightarrow \neg \mathbf{C}\left[p^{\prime}\right]\right),\left(q \sqsubseteq p^{\prime}\right), q \mathbb{Z} \pm \mathcal{X} n\right\}$, we easily get:

$\lambda x_{0} \lambda x_{1} \lambda x_{2} \lambda x_{3} \lambda x \lambda y(x)\left(x_{1}\right) y \nVdash$

$$
\forall n^{\prime} \forall p^{\prime} \forall k^{\text {ent }}\left(G\left[n^{\prime}, p^{\prime}, k\right], \vec{F}\left[n^{\prime}, p^{\prime}, f\left(n^{\prime}, p^{\prime}, k\right)\right], \neg \neg \mathrm{C}\left[p^{\prime}\right] \rightarrow \neg \neg \mathrm{C}\left[f\left(n^{\prime}, p^{\prime}, k\right)\right]\right) .
$$

It follows that $\left(\left(\xi \lambda x_{0} \lambda x_{1} \lambda x_{2} \lambda x_{3} \lambda x \lambda y(x)\left(x_{1}\right) y\right) \lambda x(x) \tau\right) \eta \nVdash \perp$, i.e. (cdc4) $\tau \xi \eta \nVdash \perp$.

From lemma 4.14, we immediately deduce $\lambda x\left(\omega_{1}\right)$ (cdc4) $x \Vdash \mathbf{C}\left[p_{0}\right], \neg \mathbf{C}\left[p^{\prime}\right] \rightarrow \perp$.

Therefore, we can put $\operatorname{dec} 0=\lambda a \lambda b \lambda x(b)(\operatorname{cdc} 4) x$.

\section{Lemma 4.15.}

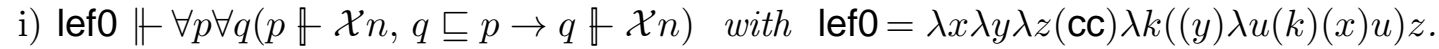

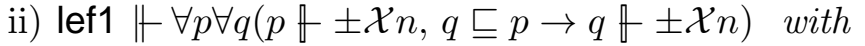

lef1 $=\lambda x \lambda y \lambda z \lambda u(($ lef0 $)(\mathrm{cc}) \lambda h((y) \lambda v(h)(x) v u) z$.

Proof.

i) This is immediate, if we write explicitly the formulas:

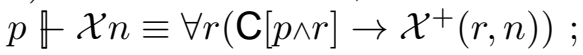

$q \sqsubseteq p \equiv \forall r(\neg \mathrm{C}[p \wedge r] \rightarrow \neg \mathrm{C}[q \wedge r]) ;$

$q \mathbb{E} \mathcal{X}_{n} \equiv \forall r\left(\mathrm{C}[q \wedge r] \rightarrow \mathcal{X}^{+}(r, n)\right)$.

We declare $x: p \Vdash \mathcal{X} n, y: q \sqsubseteq p, z: \mathrm{C}[q \wedge r], k: \neg \mathcal{X}^{+} n$.

ii) We write down the formulas:

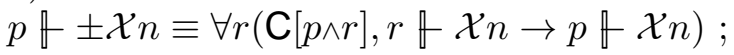


$q \sqsubseteq p \equiv \forall r(\neg \mathrm{C}[p \wedge r] \rightarrow \neg \mathrm{C}[q \wedge r]) ;$

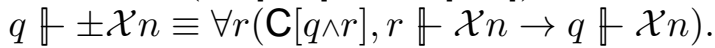

We declare $x: p \mathbb{Z} \pm \mathcal{X} n, y: q \sqsubseteq p, z: \mathrm{C}[q \wedge r], u: r \mathbb{X} n, v: \mathrm{C}[p \wedge r], h: \neg(p \Vdash \mathcal{X} n)$.

By means of lemmas 4.13)(ii) and 4.15 and also $\omega_{0} \Vdash \forall n^{\mathrm{ent}} \forall p\left(\Phi(n, p) \rightarrow p^{\prime} \sqsubseteq p\right)$, we obtain: $\lambda n \lambda x\left((\right.$ lef1)(for) $n x)\left(\omega_{0}\right) n x \Vdash \forall n^{\text {ent }} \forall q\left(\Phi(s n, q) \rightarrow p^{\prime} \mathbb{E} \pm \mathcal{X} n\right)$.

But, we have cdc1 $\Vdash \forall n^{\text {ent }} \exists p \Phi(n, p)$ (lemma 4.9) ; it follows that:

$\lambda n(\mathrm{cc}) \lambda k((\mathrm{cdc} 1)(s) n) \lambda x(k)(($ lef1 $)($ for $) n x)\left(\omega_{0}\right) n x \Vdash \forall n^{\mathrm{ent}}\left(p_{\mathcal{X}} \mathbb{E} \pm \mathcal{X} n\right)$.

Thus, we can put dec2 $=\lambda a \lambda b \lambda n(\mathrm{cc}) \lambda k((\mathrm{cdc} 1)(s) n) \lambda x(k)(($ lef1 $)($ for $) n x)(a) n x$.

This completes the proof of theorem 4.5.

\section{The Ultrafilter AXIOM ON $\mathbb{N}$}

Let us consider a standard realizability algebra $\mathcal{A}$ and a $\mathcal{A}$-model $\mathcal{M}$ in which the individual set (which is also the set of conditions) is $P=\mathcal{P}(\Pi)^{\mathbb{N}}$.

The binary relation $\varepsilon$ is defined by $\|n \varepsilon p\|=p(n)$ if $n \in \mathbb{N}$; otherwise $\|n \varepsilon p\|=\emptyset$.

$\mathbf{1}$ is defined by $\mathbf{1}(n)=\emptyset$ for every $n \in \mathbb{N}$;

$\wedge$ is defined by $\|n \varepsilon(p \wedge q)\|=\|n \varepsilon p \wedge n \varepsilon q\|$ for every $n \in \mathbb{N}$.

The axiom of representation of predicates on $\mathbb{N}(R P N)$. We define the following recursive function of arity $k$, denoted by $\left(n_{1}, \ldots, n_{k}\right)$ (coding of $k$-uples): $\left(n_{1}, n_{2}\right)=n_{1}+\left(n_{1}+\right.$ $\left.n_{2}\right)\left(n_{1}+n_{2}+1\right) / 2 ;\left(n_{1}, \ldots, n_{k+1}\right)=\left(\left(n_{1}, \ldots, n_{k}\right), n_{k+1}\right)$.

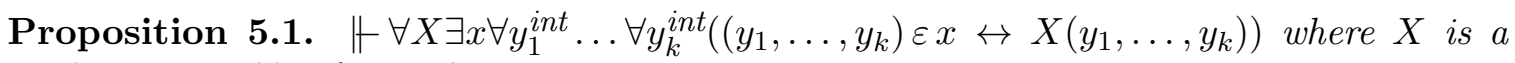
predicate variable of arity $k$.

Proof. Let $\mathcal{X}: P^{k} \rightarrow \mathcal{P}(\Pi)$ be a predicate of arity $k$. We define $a \in P$ by putting: $a(n)=\mathcal{X}\left(n_{1}, \ldots, n_{k}\right)$ for $n \in \mathbb{N}, n=\left(n_{1}, \ldots, n_{k}\right)$. Then, we have immediately:

$I \Vdash \forall y_{1}^{\text {ent }} \ldots \forall y_{k}^{\text {ent }}\left(\left(y_{1}, \ldots, y_{k}\right) \varepsilon a \rightarrow \mathcal{X}\left(y_{1}, \ldots, y_{k}\right)\right)$ and

$I \Vdash \forall y_{1}^{\text {ent }} \ldots \forall y_{k}^{\text {ent }}\left(\mathcal{X}\left(y_{1}, \ldots, y_{k}\right) \rightarrow\left(y_{1}, \ldots, y_{k}\right) \varepsilon a\right)$.

It follows that:

$\lambda x(x) I \Vdash \forall X \exists x \forall y_{1}^{\text {ent }} \ldots \forall y_{k}^{\text {ent }}\left(\left(y_{1}, \ldots, y_{k}\right) \varepsilon x \rightarrow X\left(y_{1}, \ldots, y_{k}\right)\right)$ and

$\lambda x(x) I \Vdash \forall X \exists x \forall y_{1}^{\text {ent }} \ldots \forall y_{k}^{\text {ent }}\left(X\left(y_{1}, \ldots, y_{k}\right) \rightarrow\left(y_{1}, \ldots, y_{k}\right) \varepsilon x\right)$.

Then, it suffices to apply theorem 1.13 .

The comprehension scheme for $\mathbb{N}(C S N)$. Let $F\left[y, x_{1}, \ldots, x_{k}\right]$ be a formula the free variables of which are taken among $y, x_{1}, \ldots, x_{k}$. We define a $k$-ary function $g_{F}: P^{k} \rightarrow P$, in other words $g_{F}: P^{k} \times \mathbb{N} \rightarrow \mathcal{P}(\Pi)$ by putting $g_{F}\left(p_{1}, \ldots, p_{k}\right)(n)=\left\|F\left[n, p_{1}, \ldots, p_{k}\right]\right\|$ for every $n \in \mathbb{N}$.

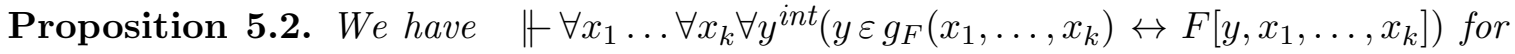
every formula $F\left[y, x_{1}, \ldots, x_{k}\right]$.

Proof. Indeed, we have trivially:

$I \Vdash \forall x_{1} \ldots \forall x_{k} \forall y^{\text {ent }}\left(y \varepsilon g_{F}\left(x_{1}, \ldots, x_{k}\right) \rightarrow F\left[y, x_{1}, \ldots, x_{k}\right]\right)$ and

$I \Vdash \forall x_{1} \ldots \forall x_{k} \forall y^{\text {ent }}\left(F\left[y, x_{1}, \ldots, x_{k}\right] \rightarrow y \varepsilon g_{F}\left(x_{1}, \ldots, x_{k}\right)\right)$.

Then, it suffices to apply theorem 1.13 . 


\section{Remark.}

The binary function symbol $\wedge$ is obtained by applying CSN to the formula $y \varepsilon x_{1} \wedge y \varepsilon x_{2}$.

The generic model. We denote by $\mathrm{C}[x]$ the formula $\forall m^{\text {int }} \exists n^{\text {int }}(m+n) \varepsilon x$, which says that the set $x$ of integers is infinite. The predicate $\mathrm{C}$ is defined by this formula: for every $p \in P,|\mathrm{C}[p]|$ is, by definition, the set $\{\tau \in \Lambda ; \tau \Vdash \mathbf{C}[p]\}$.

It follows that the condition $\gamma:: t\left(p_{1}, \ldots, p_{n}\right) \Rightarrow u\left(p_{1}, \ldots, p_{n}\right)$ is written as:

$\lambda x \gamma x \Vdash \forall p_{1} \ldots \forall p_{n}\left(\mathbf{C}\left[t\left(p_{1}, \ldots, p_{n}\right)\right] \rightarrow \mathbf{C}\left[u\left(p_{1}, \ldots, p_{n}\right)\right]\right)$.

Therefore, in order to complete the definition of the algebra $\mathcal{B}$ (and of the $\mathcal{B}$-model $\mathcal{N}$ ), it remains to find proof-like terms $\alpha_{0}, \alpha_{1}, \alpha_{2}, \beta_{0}, \beta_{1}, \beta_{2}$ such that:

$\alpha_{0} \Vdash \forall p \forall q \forall r(\mathbf{C}[(p \wedge q) \wedge r] \rightarrow \mathbf{C}[p \wedge(q \wedge r)]) ; \alpha_{1} \Vdash \forall p(\mathbf{C}[p] \rightarrow \mathbf{C}[p \wedge \mathbf{1}]) ;$

$\alpha_{2} \Vdash \forall p \forall q(\mathrm{C}[p \wedge q] \rightarrow \mathrm{C}[q]) ; \quad \beta_{0} \Vdash \forall p(\mathbf{C}[p] \rightarrow \mathrm{C}[p \wedge p]) ; \beta_{1} \Vdash \forall p \forall q(\mathrm{C}[p \wedge q] \rightarrow \mathrm{C}[q \wedge p]) ;$

$\beta_{2} \Vdash \forall p \forall q \forall r \forall s(\mathrm{C}[((p \wedge q) \wedge r) \wedge s] \rightarrow \mathrm{C}[(p \wedge(q \wedge r)) \wedge s])$.

Now, we easily have, in natural deduction:

$\vdash \theta: \forall n\left(n \varepsilon x \rightarrow n \varepsilon x^{\prime}\right) \rightarrow\left(\mathrm{C}[x] \rightarrow \mathrm{C}\left[x^{\prime}\right]\right)$ with $\theta=\lambda f \lambda u \lambda m \lambda h(u m) \lambda n \lambda x(h n)(f) x$.

Therefore, by theorem 1.3 (adequacy lemma), we can put $\alpha_{i}=\theta \alpha_{i}^{*}$ and $\beta_{i}=\theta \beta_{i}^{*}$, with proof-like terms $\alpha_{i}^{*}, \beta_{i}^{*}(0 \leq i \leq 2)$ such that:

$\vdash \alpha_{0}^{*}: \forall X \forall Y \forall Z\{(X \wedge Y) \wedge Z \rightarrow X \wedge(Y \wedge Z)\} ; \vdash \alpha_{1}^{*}: \forall X\{X \rightarrow X \wedge \top\} ; \vdash \alpha_{2}^{*}:$ $\forall X \forall Y\{X \wedge Y \rightarrow Y\} ; \vdash \beta_{0}^{*}: \forall X\{X \rightarrow X \wedge X\} ; \vdash \beta_{1}^{*}: \forall X \forall Y\{X \wedge Y \rightarrow Y \wedge X\} ;$

$\vdash \beta_{2}^{*}: \forall X \forall Y \forall Z \forall U\{((X \wedge Y) \wedge Z) \wedge U \rightarrow(X \wedge(Y \wedge Z)) \wedge U\}$.

The countable downward chain condition. In this section, we show the:

\section{Theorem 5.3.}

The forcing structure $\{\mathbf{C}, \wedge, \mathbf{1}\}$ satisfies the countable downward chain condition in $\mathcal{M}$.

Remark. The proof of this theorem is a formalization of the following simple result:

The set of infinite subsets of $\mathbb{N}$ with the preorder " $p \sqsubseteq q \Leftrightarrow p \backslash q$ is finite", satisfies the countable downward chain condition.

The proof is as follows: let $p_{n}$ be a decreasing sequence for this preorder ; put $h_{n}=\bigcap_{i \leq n} p_{i}, k_{n}=$ the first element of $h_{n}$ which is $\geq n$, and consider $\left\{k_{n} ; n \in \mathbb{N}\right\}$ which is an infinite subset of $\mathbb{N}$.

Proof. We have to find a proof-like term cdc such that:

cdc $\Vdash \forall X \exists x\left\{\forall n^{\text {ent }} \exists p X(n, p), \forall n^{\text {ent }} \forall p \forall q(X(n, p), X(n, q) \rightarrow p=q)\right.$, $\forall n^{\text {ent }} \forall p \forall q(X(n, p), X(s n, q) \rightarrow q \sqsubseteq p) \rightarrow$ $\left.\forall n^{\text {ent }} \forall p(X(n, p) \rightarrow x \sqsubseteq p) \wedge\left(\forall n^{\text {ent }} \forall p(X(n, p) \rightarrow \mathrm{C}[p]) \rightarrow \mathrm{C}[x]\right)\right\}$

where $p \sqsubseteq q$ is the formula $\forall r(\mathrm{C}[p \wedge r] \rightarrow \mathrm{C}[q \wedge r])$.

By theorem 1.13, this amounts to find a proof-like term cdc' such that:

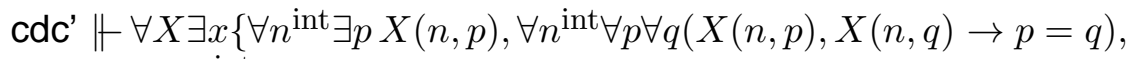
$\forall n^{\text {int }} \forall p \forall q(X(n, p), X(s n, q) \rightarrow q \sqsubseteq p) \rightarrow$ $\left.\forall n^{\mathrm{int}} \forall p(X(n, p) \rightarrow x \sqsubseteq p) \wedge\left(\forall n^{\mathrm{int}} \forall p(X(n, p) \rightarrow \mathrm{C}[p]) \rightarrow \mathrm{C}[x]\right)\right\}$.

By theorem 1.3 (adequacy lemma), given a formula $F$, we can use the following method to show $\| F$ :

First, show $\Vdash A_{1}, \ldots, \Vdash A_{k}$, then show $A_{1}, \ldots, A_{k} \vdash F$ by means of the rules of classical second order natural deduction (which contains the comprehension scheme), and of the following axioms which are realized by proof-like terms in the $\mathcal{A}$-model $\mathcal{M}$ : 
- $t \neq u$ for all closed terms $t, u$ which take distinct values in $\mathcal{M}$.

- $\forall x_{1}^{\text {int }} \ldots \forall x_{k}^{\text {int }}\left(t\left(x_{1}, \ldots, x_{k}\right)=u\left(x_{1}, \ldots, x_{k}\right)\right)$ for all the equations between terms which are true in $\mathbb{N}$.

- The foundation scheme (SCF, see theorem 1.11ii) which consists of the formulas:

$\forall X_{1} \ldots \forall X_{k}\left\{\forall x^{\text {int }}\left[\forall y^{\text {int }}\left(X_{1} y, \ldots, X_{k} y \rightarrow f(y, x) \neq 1\right), X_{1} x, \ldots, X_{k} x \rightarrow \perp\right]\right.$

$$
\left.\rightarrow \forall x^{\operatorname{int}}\left(X_{1} x, \ldots, X_{k} x \rightarrow \perp\right)\right\}
$$

where $f: P^{2} \rightarrow P$ is such that the relation $f(y, x)=1$ is well founded on $\mathbb{N}$.

- The axiom of choice scheme for individuals (ACI, see theorem 2.1) which consists of the formulas $\forall \vec{x}\left(\forall y{ }^{\text {int }} F\left(\vec{x}, f_{F}(\vec{x}, y)\right) \rightarrow \forall y F(\vec{x}, y)\right)$;

$\vec{x}=\left(x_{1}, \ldots, x_{k}\right)$ is a finite sequence of variables, $\forall \vec{x} \forall y^{\text {int }} F$ is an arbitrary closed formula, and $f_{F}$ is a function symbol of arity $k+1$.

- The axiom of representation of predicates on $\mathbb{N}(\mathrm{RPN}$, see proposition [5.1) which consists of the formulas $\forall X \exists x \forall \vec{y}^{\text {int }}\left(\left(y_{1}, \ldots, y_{k}\right) \varepsilon x \leftrightarrow X \vec{y}\right)$;

$\vec{y}=\left(y_{1}, \ldots, y_{k}\right)$ is a sequence of $k$ variables and $X$ is a predicate variable of arity $k$.

- The comprehension scheme for integers (CSN, see proposition 5.2), which consists of the formulas $\forall \vec{x} \forall y^{\text {int }}\left(y \in g_{F}(\vec{x}) \leftrightarrow F[y, \vec{x}]\right)$;

$\vec{x}=\left(x_{1}, \ldots, x_{k}\right)$ is a sequence of $k$ variables, $\forall \vec{x} \forall y^{\text {int }} F$ is an arbitrary closed formula, and $g_{F}$ is a function symbol of arity $k$.

Lemma 5.4. $\vdash \forall p \forall q\left(p \sqsubseteq q \leftrightarrow \exists m^{i n t} \forall n^{i n t}(n+m \varepsilon p \rightarrow n+m \varepsilon q)\right)$.

Proof. We apply the CSN to the formula $F[y, x] \equiv y \notin x$; thus, we obtain:

using the notation $\neg x$ for $g_{F}(x)$.

$$
\vdash \forall x \forall y^{\operatorname{int}}(y \varepsilon \neg x \leftrightarrow y \notin x)
$$

We have $p \sqsubseteq q \equiv \forall r(\mathrm{C}[p \wedge r] \rightarrow \mathrm{C}[q \wedge r])$ and therefore $p \sqsubseteq q \vdash \mathrm{C}[p \wedge \neg q] \rightarrow \mathrm{C}[q \wedge \neg q]$.

But, we have $\mathrm{C}[q \wedge \neg q] \vdash \forall m^{\text {int }} \exists n^{\text {int }}(m+n \varepsilon q \wedge m+n \notin q) \vdash \perp$, and thus:

$p \sqsubseteq q \vdash \neg \mathrm{C}[p \wedge \neg q]$, that is $\vdash p \sqsubseteq q \rightarrow \exists m^{\text {int }} \forall n^{\text {int }} \neg(m+n \varepsilon p \wedge \neg(m+n \varepsilon q))$.

Conversely, from the hypothesis:

$\forall n^{\prime \text { int }}\left(m^{\prime}+n^{\prime} \varepsilon p \rightarrow m^{\prime}+n^{\prime} \varepsilon q\right), \forall m^{\text {int }} \exists n^{\text {int }}(m+n \varepsilon p \wedge m+n \varepsilon r)$, we deduce:

$\forall m^{\operatorname{int}} \exists n^{\operatorname{int}}\left(\left(m^{\prime}+m\right)+n \varepsilon p \wedge\left(m^{\prime}+m\right)+n \varepsilon r\right)$, then:

$\forall m^{\text {int }} \exists n^{\text {int }}\left(m+\left(m^{\prime}+n\right) \varepsilon q \wedge m+\left(m^{\prime}+n\right) \varepsilon r\right)$ then:

$\forall m^{\text {int }} \exists n^{\text {int }}(m+n \varepsilon q \wedge m+n \varepsilon r)$. Therefore:

$\forall n^{\prime \text { int }}\left(m^{\prime}+n^{\prime} \varepsilon p \rightarrow m^{\prime}+n^{\prime} \varepsilon q\right) \vdash \mathrm{C}[p \wedge r] \rightarrow \mathrm{C}[q \wedge r]$ and thus:

$\exists m^{\prime} \forall n^{\prime \text { int }}\left(m^{\prime}+n^{\prime} \varepsilon p \rightarrow m^{\prime}+n^{\prime} \varepsilon q\right) \vdash \mathrm{C}[p \wedge r] \rightarrow \mathrm{C}[q \wedge r]$.

Applying RPN and the comprehension scheme, we obtain $\Vdash \forall X \exists h D(h, X)$ with: $D(h, X) \equiv \forall k^{\text {int }} \forall n^{\text {int }}\left((k, n) \varepsilon h \leftrightarrow \forall q \forall i^{\text {int }}(i \leq n, X(i, q) \rightarrow k \varepsilon q)\right)$.

Remark. The intuitive meaning of $D(h, X)$ is: $h$ is the individual associated with the decreasing sequence of conditions $X^{\prime}$, the $n$-th term of which is the intersection of the $n$ first terms of the sequence $X$.

We apply CSN to the formula $F(k, n, h) \equiv(k, n) \varepsilon h$. Thus, we obtain:

$\vdash \forall n \forall h \forall k^{\text {int }} \forall n\left(k \varepsilon g_{F}(n, h) \leftrightarrow(k, n) \varepsilon h\right)$.

We shall use the notation $h_{n}$ for $g_{F}(n, h)$. Therefore, we have:

$$
\vdash \forall n \forall h \forall k^{\text {int }}\left(k \varepsilon h_{n} \leftrightarrow(k, n) \varepsilon h\right) .
$$

and it follows that:

$$
D(h, X) \vdash \forall k^{\mathrm{int}} \forall n^{\mathrm{int}}\left(k \varepsilon h_{n} \leftrightarrow \forall q \forall i^{\mathrm{int}}(i \leq n, X(i, q) \rightarrow k \varepsilon q)\right)
$$


We put $\Phi(k, h, n) \equiv \exists i^{\operatorname{int}}\left\{\forall j^{\operatorname{int}}\left(j+n \varepsilon h_{n} \rightarrow(j<i) \neq 1\right), i+n \varepsilon h_{n}, k=i+n\right\}$.

Remark. The intuitive meaning of $\Phi(k, h, n)$ is: " $k$ is the first element of $h_{n}$ which is $\geq n$ ".

We apply CSN to the formula $F(k, h) \equiv \exists n^{\text {int }} \Phi(k, h, n)$. Thus, we obtain:

$\vdash \forall h \forall k^{\mathrm{int}}\left(k \varepsilon g_{F}(h) \leftrightarrow \exists n^{\mathrm{int}} \Phi(k, h, n)\right)$.

We shall use the notation $\inf (h)$ for $g_{F}(h)$. Therefore, we have:

$$
\vdash \forall h \forall k^{\operatorname{int}}\left(k \varepsilon \inf (h) \leftrightarrow \exists n^{\operatorname{int}} \Phi(k, h, n)\right) .
$$

The hypothesis of the c.d.c. are:

$H_{0}[X] \equiv \forall n^{\mathrm{int}} \exists p X(n, p)$;

$H_{1}[X] \equiv \forall n^{\text {int }} \forall p \forall q(X(n, p), X(n, q) \rightarrow p=q) ;$

$H_{2}[X] \equiv \forall n^{\operatorname{int}} \forall p \forall q(X(n, p), X(s n, q) \rightarrow q \sqsubseteq p)$;

$H_{3}[X] \equiv \forall n^{\text {int }} \forall p(X(n, p) \rightarrow \mathrm{C}[p])$.

We put $\vec{H}[X] \equiv\left\{H_{0}[X], H_{1}[X], H_{2}[X], H_{3}[X]\right\}$ and $\vec{H}_{*}[X]=\left\{H_{0}[X], H_{1}[X], H_{2}[X]\right\}$.

Thus, it is sufficient to show:

$D(h, X), \vec{H}_{*}[X] \vdash \forall n^{\text {int }} \forall p(X(n, p) \rightarrow \inf (h) \sqsubseteq p)$ and

$D(h, X), \vec{H}[X] \vdash \mathrm{C}[\inf (h)]$.

Notation. The formula $\forall n^{\text {int }}(n \varepsilon p \rightarrow n \varepsilon q)$ is denoted by $p \subseteq q$.

Lemma 5.5. $D(h, X) \vdash \forall m^{i n t} \forall n^{i n t}\left(h_{n+m} \subseteq h_{n}\right)$.

Proof. This formula is written $\forall m^{\text {int }} \forall n^{\text {int }} \forall k^{\text {int }}\left(k \varepsilon h_{n+m} \rightarrow k \varepsilon h_{n}\right)$. Now, we have:

$D(h, X) \vdash \forall m^{\text {int }} \forall n^{\text {int }} \forall k^{\text {int }}\left(k \varepsilon h_{n+m} \rightarrow \forall q \forall i^{\text {int }}(i \leq n+m, X(i, q) \rightarrow k \varepsilon q)\right) ;$

$\vdash \forall m^{\text {int }} \forall n^{\text {int }} \forall k^{\text {int }}\left[\forall q \forall i^{\text {int }}(i \leq n+m, X(i, q) \rightarrow k \varepsilon q) \rightarrow \forall q \forall i^{\text {int }}(i \leq n, X(i, q) \rightarrow k \varepsilon q)\right]$ :

$D(h, X) \vdash \forall m^{\text {int }} \forall n^{\text {int }} \forall k^{\text {int }}\left(\forall q \forall i^{\text {int }}(i \leq n, X(i, q) \rightarrow k \varepsilon q) \rightarrow k \varepsilon h_{n}\right)$.

Lemma 5.6. $D(h, X), H_{0}[X], H_{1}[X] \vdash \forall n^{i n t} \forall k^{i n t} \forall p\left(X(s n, p), k \varepsilon p, k \varepsilon h_{n} \rightarrow k \varepsilon h_{s n}\right)$.

Proof. We have $D(h, X), \operatorname{int}(k), \operatorname{int}(n) \vdash \forall p \forall i^{\operatorname{int}}(i \leq s n, X(i, p) \rightarrow k \varepsilon p) \rightarrow k \varepsilon h_{s n}$.

But, we have $\operatorname{int}(n), \operatorname{int}(i), i \leq s n \vdash i \leq n \vee i=s n$, and therefore:

$\operatorname{int}(n), \forall p \forall i^{\operatorname{int}}(i \leq n, X(i, p) \rightarrow k \varepsilon p), \forall p(X(s n, p) \rightarrow k \varepsilon p) \vdash$

$$
\forall p \forall i^{\mathrm{int}}(i \leq s n, X(i, p) \rightarrow k \varepsilon p) .
$$

It follows that:

$D(h, X), \operatorname{int}(k), \operatorname{int}(n) \vdash \forall p \forall i^{\operatorname{int}}(i \leq n, X(i, p) \rightarrow k \varepsilon p), \forall p(X(s n, p) \rightarrow k \varepsilon p) \rightarrow k \varepsilon h_{s n}$, i.e.:

$D(h, X), \operatorname{int}(k), \operatorname{int}(n) \vdash k \varepsilon h_{n}, \forall p(X(s n, p) \rightarrow k \varepsilon p) \rightarrow k \varepsilon h_{s n}$. Therefore:

$D(h, X), \operatorname{int}(k), \operatorname{int}(n), H_{0}[X], H_{1}[X] \vdash \forall p\left(k \varepsilon h_{n}, X(s n, p), k \varepsilon p \rightarrow k \varepsilon h_{s n}\right)$.

Lemma 5.7. $D(h, X), \vec{H}_{*}[X] \vdash \forall n^{i n t} \forall p\left(X(n, p) \rightarrow p \sqsubseteq h_{n}\right)$.

Proof. By recurrence on $n$. We must show:

$D(h, X), \vec{H}_{*}[X], \operatorname{int}(n) \vdash \forall p \exists m^{\operatorname{int}} \forall l^{\operatorname{int}}\left(X(n, p), l+m \varepsilon p \rightarrow l+m \varepsilon h_{n}\right)$.

For $n=0$, we have $D(h, X) \vdash \forall k^{\text {int }}\left(\forall q(X(0, q) \rightarrow k \varepsilon q) \rightarrow k \varepsilon h_{0}\right)$. Thus, it suffices to show:

$D(h, X), \vec{H}_{*}[X] \vdash \forall p \exists m^{\text {int }} \forall l^{\text {int }} \forall q(X(0, p), l+m \varepsilon p, X(0, q) \rightarrow l+m \varepsilon q)$,

which follows, in fact, from $H_{1}[X]$, that is $X(0, p), X(0, q) \rightarrow p=q$.

The recurrence hypothesis is $\forall p\left(X(n, p) \rightarrow p \sqsubseteq h_{n}\right)$;

$H_{2}[X]$ is $\forall p \forall q(X(n, p), X(s n, q) \rightarrow q \sqsubseteq p) ; H_{0}[X]$ is $\exists p X(n, p)$.

Moreover, we have easily $q \sqsubseteq p, p \sqsubseteq r \vdash q \sqsubseteq r$. Thus, it follows that:

$\forall p\left(X(s n, p) \rightarrow p \sqsubseteq h_{n}\right)$, i.e. $\forall p \exists m^{\text {int }} \forall l^{\text {int }}\left(X(s n, p), l+m \varepsilon p \rightarrow l+m \varepsilon h_{n}\right)$. 
Now, we have, by lemma 5.6 . $D(h, X), H_{0}[X], H_{1}[X] \vdash X(s n, p), l+m \varepsilon p, l+m \varepsilon h_{n} \rightarrow l+m \varepsilon h_{s n}$. Therefore, we have $\forall p \exists m^{\text {int }} \forall l^{\text {int }}\left(X(s n, p), l+m \varepsilon p \rightarrow l+m \varepsilon h_{s n}\right)$ that is: $\forall p\left(X(s n, p) \rightarrow p \sqsubseteq h_{s n}\right)$, which is the desired result.

Lemma 5.8. $D(h, X), \vec{H}(X) \vdash \forall n^{i n t} \mathrm{C}\left[h_{n}\right]$.

Proof. We have $\forall n^{\text {int }} \forall p(X(n, p) \rightarrow \mathrm{C}[p])$ from $H_{3}$. Moreover, we have easily: $\vdash \forall p \forall q(\mathrm{C}[p], p \sqsubseteq q \rightarrow \mathrm{C}[q])$. Thus, applying lemma 5.7, we obtain: $D(h, X), \vec{H}(X) \vdash \forall n^{\text {int }} \forall p\left(X(n, p) \rightarrow \mathrm{C}\left[h_{n}\right]\right)$. Hence the result, from $H_{0}[X]$.

Lemma 5.9. $D(h, X), \vec{H}[X] \vdash \forall n^{i n t} \exists k^{i n t} \Phi(k, h, n)$.

Proof. By the foundation scheme (SCF), we have:

$\vdash \forall i^{\text {int }}\left\{\forall j^{\text {int }}\left(j+n \varepsilon h_{n} \rightarrow(j i i) \neq 1\right), i+n \varepsilon h_{n} \rightarrow \perp\right\} \rightarrow \forall i^{\text {int }}\left(i+n \varepsilon h_{n} \rightarrow \perp\right)$.

But, we have $D(h, X), \vec{H}[X] \vdash \forall n^{\text {int }} \mathrm{C}\left[h_{n}\right]$ (lemma 5.8), therefore:

$D(h, X), \vec{H}[X] \vdash \forall n^{\text {int }} \exists i^{\text {int }} i+n \varepsilon h_{n}$. It follows that:

$D(h, X), \vec{H}[X] \vdash \forall n^{\text {int }} \exists i^{\text {int }}\left\{\forall j^{\text {int }}\left(j+n \varepsilon h_{n} \rightarrow\left(j_{i} i\right) \neq 1\right), i+n \varepsilon h_{n}\right\}$.

Lemma 5.10. $D(h, X), \vec{H}[X] \vdash \mathrm{C}[\inf (h)]$.

Proof. We have $\mathrm{C}[\inf (h)] \equiv \forall m^{\operatorname{int}} \exists i^{\operatorname{int}}(i+m \varepsilon \inf (h))$.

Now, by definition of the function symbol inf, we have:

$\vdash \forall h \forall k^{\operatorname{int}}\left(k \varepsilon \inf (h) \leftrightarrow \exists n^{\operatorname{int}} \Phi(k, h, n)\right)$.

Therefore $\vdash \mathrm{C}[\inf (h)] \leftrightarrow \forall m^{\operatorname{int}} \exists i^{\operatorname{int}} \exists n^{\operatorname{int}} \Phi(i+m, h, n)$.

By definition de $\Phi$, we have trivially $\vdash \forall n^{\text {int }} \forall k^{\text {int }}\left(\Phi(k, h, n) \rightarrow \exists i^{\text {int }}(k=i+n)\right)$.

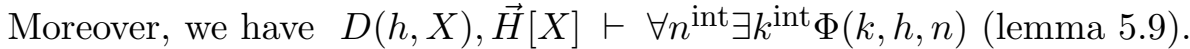

Therefore $D(h, X), \vec{H}[X] \vdash \forall n^{\text {int }} \exists i^{\text {int }} \Phi(i+n, h, n)$, thus $D(h, X), \vec{H}[X] \vdash \mathrm{C}[\inf (h)]$.

Lemma 5.11.

$D(h, X), \vec{H}_{*}[X] \vdash \forall h \forall k^{i n t} \forall k^{\prime i n t} \forall n^{i n t} \forall n^{\prime i n t}\left(\Phi(k, h, n), \Phi\left(k^{\prime}, h, n^{\prime}\right), k^{\prime}>k \rightarrow n^{\prime}>n\right)$.

Proof. We have $\Phi(k, h, n) \equiv \exists i^{\mathrm{int}} \vec{\Psi}(k, h, n, i)$, with :

$\vec{\Psi}(k, h, n, i) \equiv\left\{\forall j^{\mathrm{int}}\left(j+n \varepsilon h_{n} \rightarrow(j i i) \neq 1\right), i+n \varepsilon h_{n}, k=i+n\right\}$.

Thus, we have to show:

$D(h, X), \vec{H}_{*}[X], \operatorname{int}(k), \operatorname{int}\left(k^{\prime}\right), \operatorname{int}(n), \operatorname{int}\left(n^{\prime}\right), \operatorname{int}(i), \operatorname{int}\left(i^{\prime}\right) \vdash \vec{\Xi}\left(h, k, n, i, k^{\prime}, n^{\prime}, i^{\prime}\right) \rightarrow \perp$

with $\vec{\Xi}\left(h, k, n, i, k^{\prime}, n^{\prime}, i^{\prime}\right) \equiv\left\{\vec{\Psi}(k, h, n, i), \vec{\Psi}\left(k^{\prime}, h, n^{\prime}, i^{\prime}\right), k^{\prime}>k, n^{\prime} \leq n\right\}$ that is:

$\vec{\Xi}\left(h, k, n, i, k^{\prime}, n^{\prime}, i^{\prime}\right) \equiv$

$\left\{\forall j^{\operatorname{int}}\left(j+n \varepsilon h_{n} \rightarrow\left(j_{i} i\right) \neq 1\right), i+n \varepsilon h_{n}, k=i+n\right.$,

$\forall j^{\prime \operatorname{int}}\left(j^{\prime}+n^{\prime} \varepsilon h_{n^{\prime}} \rightarrow\left(j^{\prime} i i^{\prime}\right) \neq 1\right), i^{\prime}+n^{\prime} \varepsilon h_{n^{\prime}}, k^{\prime}=i^{\prime}+n^{\prime}$,

$\left.k^{\prime}>k, n^{\prime} \leq n\right\}$.

From $n^{\prime} \leq n$ and $k=i+n$, we deduce $n^{\prime} \leq k$, thus $k=j^{\prime}+n^{\prime}$.

From $k^{\prime}>k$, we deduce $i^{\prime}+n^{\prime}>k$, and thus $j^{\prime}<i^{\prime}$.

Therefore, we have $j^{\prime}+n^{\prime} \notin h_{n^{\prime}}$, i.e. $k \notin h_{n^{\prime}}$. But, from $n^{\prime} \leq n$, we deduce $h_{n} \subseteq h_{n^{\prime}}$ (lemma 5.5), thus $k \notin h_{n}$, which contradicts $i+n \varepsilon h_{n}, k=i+n$.

By definition of $\Phi$, we have trivially $\vdash \forall n^{\operatorname{int}} \forall k^{\text {int }}\left(\Phi(k, h, n) \rightarrow k \varepsilon h_{n}\right)$.

By lemmas 5.5 and 5.11, we get:

$D(h, X), \vec{H}_{*}[X] \vdash \forall h \forall k^{\text {int }} \forall k^{\prime \text { int }} \forall n^{\text {int }} \forall n^{\prime \text { int }}\left(\Phi(k, h, n), \Phi\left(k^{\prime}, h, n^{\prime}\right), k^{\prime}>k \rightarrow k^{\prime} \varepsilon h_{n}\right)$.

Lemma 5.9 gives $\forall n^{\text {int }} \exists k^{\text {int }} \Phi(k, h, n)$. It follows that: 
$D(h, X), \vec{H}_{*}[X] \vdash \forall n^{\text {int }} \exists k^{\text {int }} \forall n^{\prime \text { int }} \forall k^{\prime \text { int }}\left(\Phi\left(k^{\prime}, h, n^{\prime}\right), k^{\prime}>k \rightarrow k^{\prime} \varepsilon h_{n}\right)$,

and therefore $D(h, X), \vec{H}_{*}[X] \vdash \forall n^{\operatorname{int}}\left(\inf (h) \sqsubseteq h_{n}\right)$.

But, we have trivially $D(h, X) \vdash \forall n^{\text {int }} \forall k^{\text {int }} \forall p\left(k \varepsilon h_{n}, X(n, p) \rightarrow k \varepsilon p\right)$. Therefore, finally:

$D(h, X), \vec{H}_{*}[X] \vdash \forall n^{\text {int }} \forall p(X(n, p) \rightarrow \inf (h) \sqsubseteq p)$.

We have eventually obtained the desired proof-like term cdc', which completes the proof of theorem 5.3 .

The ultrafilter. In the model $\mathcal{N}$, we have defined the generic ideal $\mathcal{J}$, which is a unary predicate, by putting: $\mathcal{J}(p)=\Pi \times\{p\}$ for every $p \in P$.

By theorem 3.5, we have:

i) $\| \vdash \neg \mathcal{J}(\mathbf{1})$

ii) $\| \Vdash \forall x(\neg \mathrm{C}[x] \rightarrow \mathcal{J}(x))$

iii) $\| \models \forall x \forall y(\mathcal{J}(x \wedge y) \rightarrow \mathcal{J}(x) \vee \mathcal{J}(y))$

iv) $\| \leftarrow \forall x(\forall y(\neg \mathrm{C}[x \wedge y] \rightarrow \mathcal{J}(y)) \rightarrow \neg \mathcal{J}(x))$

v) $\| \leftarrow \forall x \forall y(\mathcal{J}(x), y \sqsubseteq x \rightarrow \mathcal{J}(y))$

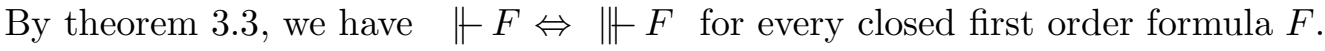

Remark. A "first order" formula contains quantifiers on the individuals which, by means of the symbol $\varepsilon$, represent the subsets of $\mathbb{N}$. Therefore, it is a second order formula from the point of view of Arithmetic. But it contains no quantifier on sets of individuals.

By theorems 1.13 and 2.13, we can use, in $F$, the quantifier $\forall x^{\text {int }}$, since the quantifier $\forall x^{\text {ent }}$ is first order.

Therefore, we have:

vi) $\|-\mathrm{C}[x] \leftrightarrow \forall m^{\text {int }} \exists n^{\text {int }}(m+n \varepsilon x)$

vii) $\| t y \sqsubseteq x \leftrightarrow \exists m^{\text {int }} \forall n^{\text {int }}(m+n \varepsilon y \rightarrow m+n \varepsilon x)$

viii) $\left\|\Vdash \forall n^{\text {int }} n \varepsilon \mathbf{1} ;\right\| \nVdash \forall x \forall y \forall n^{\text {int }}(n \varepsilon x \wedge y \leftrightarrow n \varepsilon x \wedge n \varepsilon y)$

since all these formulas are first order. Properties (i) to (viii) show that, in the $\mathcal{B}$-model $\mathcal{N}$, the following formula is realized:

$\mathcal{J}$ is a maximal non trivial ideal on the Boolean algebra of the subsets of $\mathbb{N}$ which are represented by individuals.

Now, by theorems 4.4 and 5.3 , the following formula is realized in $\mathcal{N}$ :

Every subset of $\mathbb{N}$ is represented by an individual.

Thus the following formula is realized in $\mathcal{N}$ :

$\mathcal{J}$ is a maximal non trivial ideal on the Boolean algebra of the subsets of $\mathbb{N}$.

Programs obtained from proofs. Let $F$ be a formula of second order arithmetic, that is to say a second order formula every individual quantifier of which is restricted to $\mathbb{N}$ and every second order quantifier of which is restricted to $\mathcal{P}(\mathbb{N})$.

We associate with $F$, a first order formula $F^{\dagger}$, defined by recurrence on $F$ :

- If $F$ is $t=u, F^{\dagger} \equiv F$.

- If $F$ is $X t, F^{\dagger}$ is $t \varepsilon X^{-}$, where $X^{-}$is an individual variable associated with the unary predicate variable $X$.

- If $F$ is $A \rightarrow B, F^{\dagger}$ is $A^{\dagger} \rightarrow B^{\dagger}$. 
- If $F$ is $\forall x A, F^{\dagger}$ is $\forall x^{\text {int }} A^{\dagger}$.

- If $F$ is $\forall X A, F^{\dagger}$ is $\forall X^{-} A^{\dagger}$.

We note that, if $F$ is a formula of first order arithmetic, then $F^{\dagger}$ is simply the restriction $F^{\text {int }}$ of $F$ to the predicate $\operatorname{int}(x)$.

Let $F$ be a closed formula of second order arithmetic and let us consider a proof of $F$, which uses the axiom of dependent choice DC and the axiom UA of ultrafilter on $\mathbb{N}$, written in the following form, with a constant $\mathcal{J}$ of predicate: " $\mathcal{J}$ is a maximal non trivial ideal on $\mathcal{P}(\mathbb{N})$ ".

We can transform it immediately into a proof of $F^{\dagger}$ if we add the axiom RPN of representation of predicates on $\mathbb{N}: \forall X \exists x \forall y(y \varepsilon x \leftrightarrow X y)$. Thus, we obtain:

$x: \mathrm{UA}, y: \mathrm{RPN}, z: \mathrm{DC}^{\dagger} \vdash t[x, y, z]: F^{\dagger}$.

Therefore, we have $\vdash u: \mathrm{UA}, \mathrm{RPN} \rightarrow G$ with $u=\lambda x \lambda y \lambda z t[x, y, z]$ and $G \equiv \mathrm{DC}^{\dagger} \rightarrow F^{\dagger}$.

Thus, $G$ is a first order formula.

In the previous section, we obtained proof-like terms $\theta, \theta^{\prime}$ such that $(\theta, \mathbf{1}) \| \in U A$ and $\left(\theta^{\prime}, \mathbf{1}\right) \| \leftarrow$ RPN (theorems 4.4 and 5.31$)$.

Therefore, theorem 2.11 (adequacy lemma) gives $\left(u^{*}, \mathbf{1}_{u}\right)(\theta, \mathbf{1})\left(\theta^{\prime}, \mathbf{1}\right) \|-G$, that is to say: $\left(v,\left(\mathbf{1}_{u} \wedge \mathbf{1}\right) \wedge \mathbf{1}\right) \|-G$ with $v=\left(\left(\bar{\alpha}_{0}\right)\left(\bar{\alpha}_{0}\right) u^{*} \theta\right) \theta^{\prime}$.

By theorem $\left[3.3\right.$, we thus have $\delta_{G}^{\prime} v \Vdash \mathrm{C}\left[\left(\mathbf{1}_{u} \wedge \mathbf{1}\right) \wedge \mathbf{1}\right] \rightarrow G$, that is:

$\delta_{G}^{\prime} v \Vdash \mathrm{C}\left[\left(\mathbf{1}_{u} \wedge \mathbf{1}\right) \wedge \mathbf{1}\right], \mathrm{DC}^{\dagger} \rightarrow F$.

The axiom $\mathrm{DC}^{\dagger}$ is consequence of ACI (axiom of choice for individuals). Therefore, by theorem 2.1, we have a proof-like term $\eta_{0} \Vdash \mathrm{DC}^{\dagger}$.

Moreover, we have obviously a proof-like term $\xi_{0} \Vdash \mathrm{C}\left[\left(\mathbf{1}_{u} \wedge \mathbf{1}\right) \wedge \mathbf{1}\right]$.

Thus, finally, we have $\delta_{G}^{\prime} v \xi_{0} \eta_{0} \Vdash F$.

Then, we can apply to the program $\zeta=\delta_{G}^{\prime} v \xi_{0} \eta_{0}$ all the results obtained in the framework of usual classical realizability. The case when $F$ is an arithmetical (resp. $\Pi_{1}^{1}$ ) formula is considered in [13] (resp. [14]).

Let us take two very simple examples:

If $F \equiv \forall X(X 1, X 0 \rightarrow X 1)$, we have $\zeta \star \kappa \bullet \kappa^{\prime} \cdot \pi \succ \kappa \star \pi$ for all terms $\kappa, \kappa^{\prime} \in \Lambda$ and every stack $\pi \in \Pi$.

If $F \equiv \forall m^{\text {int }} \exists n^{\operatorname{int}}(\phi(m, n)=0)$, where $\phi$ is a function symbol, then for every $m \in \mathbb{N}$, there exists $n \in \mathbb{N}$ such that $\phi(m, n)=0$ and $\zeta \star \underline{m} \cdot T \kappa \cdot \pi \succ \kappa \star \underline{n} \cdot \pi^{\prime}$.

$T$ is the proof-like term for integer storage, given in theorem 1.13(i).

$\pi, \kappa$ are arbitrary ; therefore, by taking a constant for $\kappa$, we obtain a program which computes $n$ from $m$.

\section{WELL ORDERING ON $\mathbb{R}$}

The $\mathcal{A}$-model $\mathcal{M}$ is the same as in the previous section: the set of individuals is $P=\mathcal{P}(\Pi)^{\mathbb{N}}$. Recall that an element of $P$ is called sometimes an individual, sometimes a condition, depending on the context.

We put $(m, n)=m+(m+n)(m+n+1) / 2$ (bijection of $\mathbb{N}^{2}$ onto $\left.\mathbb{N}\right)$. We define a binary function $\gamma: P^{2} \rightarrow P$ by putting: $\gamma(n, p)(i)=p(i, n)$ if $n \in \mathbb{N} ; \gamma(n, p)$ is arbitrary (for instance 0 ) if $n \notin \mathbb{N}$.

Notation. In the sequel, we shall write $p_{n}$ instead of $\gamma(n, p)$. Thus, it is the same to give an individual $p$ or a sequence of individuals $p_{n}(n \in \mathbb{N})$.

If $i, n \in \mathbb{N}$, we have $\|(i, n) \varepsilon p\|=\left\|i \varepsilon p_{n}\right\|$. 
We fix a well ordering $\triangleleft$ on $P=\mathcal{P}(\Pi)^{\mathbb{N}}$, which is strict (i.e. $\forall x \neg(x \triangleleft x)$ ) and isomorphic to the cardinal $2^{\aleph_{0}}$ : every proper initial segment of $\triangleleft$ is therefore of power $<2^{\aleph_{0}}$. We define a binary function, denoted by $(p \triangleleft q)$ by putting $(p \triangleleft q)=1$ if $p \triangleleft q ; \quad(p \triangleleft q)=0$ otherwise. Since the relation $(p \triangleleft q)=1$ is well founded on $P$, we have (theorem 1.11): $\mathrm{Y} \Vdash \forall X[\forall x(\forall y((y \triangleleft x)=1 \mapsto X y) \rightarrow X x) \rightarrow \forall x X x]$ in the $\mathcal{A}$-model $\mathcal{M}$, but also in every $\mathcal{B}$-model $\mathcal{N}$.

We shall write, in abridged form, $y \triangleleft x$ for $(y \triangleleft x)=1$.

Thus, in $\mathcal{M}$ and $\mathcal{N}$, the relation $\triangleleft$ is well founded but, in general, not total.

It is a strict order relation, in both models ; indeed we have immediately, in the model $\mathcal{M}$ : $I \Vdash \forall x((x \triangleleft x) \neq 1) ; I \Vdash \forall x \forall y \forall z((x \triangleleft y)=1 \mapsto((y \triangleleft z)=1 \mapsto(x \triangleleft z)=1))$.

Since all these formulas are first order, by theorem 3.3, we have also, in the model $\mathcal{N}$ : $\|\leftarrow \forall x((x \triangleleft x) \neq 1) ; \quad\| \Vdash \forall x \forall y \forall z((x \triangleleft y)=1 \mapsto((y \triangleleft z)=1 \mapsto(x \triangleleft z)=1))$.

A condition $p \in P$ is also a sequence of individuals $p_{k}$. Intuitively, we shall consider it, as " the set of individuals $p_{k+1}$ for $k \varepsilon p_{0}$ " ; we define accordingly the condition $\mathbf{1}$, the formula $\mathrm{C}[p]$ which says that $p$ is a non trivial condition, and the binary operation $\wedge$.

$\mathbf{1}$ is the empty set, in other words $i \varepsilon \mathbf{1}_{0}$ (i.e. $\left.(i, 0) \varepsilon \mathbf{1}\right)$ must be false. Therefore, we put: $\mathbf{1}(n)=\Pi$ for every $n \in \mathbb{N}$.

A condition is non trivial if the set of individuals, which is associated with it, is totally ordered by $\triangleleft$. Therefore, we put:

$\mathrm{C}[p] \equiv \forall i^{\text {ent }} \forall j{ }^{\text {ent }}\left(i \varepsilon p_{0}, j \varepsilon p_{0} \rightarrow E\left[p_{i+1}, p_{j+1}\right]\right)$ with :

$E[x, y] \equiv(x=y \vee x \triangleleft y \vee y \triangleleft x)$ that is $E[x, y] \equiv(x \neq y,(x \triangleleft y) \neq 1,(y \triangleleft x) \neq 1 \rightarrow \perp)$.

The set associated with $p \wedge q$ is the union of the sets associated with $p$ and with $q$; therefore, we put:

$p \wedge q=r$ where $r_{0}$ is defined by: $\left\|2 i \varepsilon r_{0}\right\|=\left\|i \varepsilon p_{0}\right\| ;\left\|2 i+1 \varepsilon r_{0}\right\|=\left\|i \varepsilon q_{0}\right\|$;

$r_{j+1}$ is defined by: $r_{2 i+1}=p_{i+1} ; r_{2 i+2}=q_{i+1}$.

The notation $p \subset q$ means that the set associated with $q$ contains the one associated with $p$.

Therefore, we put:

$p \subset q \equiv \forall i^{\mathrm{ent}}\left(i \varepsilon p_{0} \rightarrow \exists j^{\mathrm{ent}}\left\{j \varepsilon q_{0}, p_{i+1}=q_{j+1}\right\}\right)$.

\section{Lemma 6.1.}

i) $\theta \Vdash \forall p \forall q \forall r(p \subset q, q \subset r \rightarrow p \subset r)$ with $\theta=\lambda f \lambda g \lambda i \lambda x \lambda h(f i x) \lambda j \lambda y(g) j y h$.

ii) $\theta^{\prime} \Vdash \forall p \forall q \forall r(p \subset q \rightarrow p \wedge r \subset q \wedge r)$ with:

$\theta^{\prime}=\lambda f \lambda i \lambda y \lambda u((e i)(u) i y)\left(\left((f)\left(d_{2}\right) i y\right) \lambda j(u)\left(d_{0}\right) j\right.$

where $d_{0}, d_{1}, d_{2}$, e are proof-like terms representing respectively the recursive functions:

$n \mapsto 2 n, n \mapsto 2 n+1, n \mapsto[n / 2], n \mapsto$ parity of $n$ (e returns boolean values).

Proof.

i) We suppose:

$f \nVdash \forall i\left(\operatorname{ent}(i), i \varepsilon p_{0}, \forall j\left(\operatorname{ent}(j), j \varepsilon q_{0} \rightarrow p_{i+1} \neq q_{j+1}\right) \rightarrow \perp\right)$;

$g \Vdash \forall j\left(\operatorname{ent}(j), j \varepsilon q_{0}, \forall k\left(\operatorname{ent}(k), k \varepsilon r_{0} \rightarrow q_{j+1} \neq r_{k+1}\right) \rightarrow \perp\right)$;

$x \Vdash i \varepsilon p_{0} ; h \| \forall k\left(\operatorname{ent}(k), k \varepsilon r_{0} \rightarrow p_{i+1} \neq r_{k+1}\right) ;$ and we have $\underline{i} \in|\operatorname{ent}(i)|$.

It follows that $f \underline{i} x \Vdash \forall j\left(\operatorname{ent}(j), j \varepsilon q_{0} \rightarrow p_{i+1} \neq q_{j+1}\right) \rightarrow \perp$.

Suppose that $y \nVdash j \varepsilon q_{0}$ and let $\underline{j} \in|\operatorname{ent}(j)|$.

If $p_{i+1}=q_{j+1}$, then $g j y h \Vdash \perp$; therefore $g j y h \Vdash p_{i+1} \neq q_{j+1}$. We have shown:

$\lambda j \lambda y(g) j y h \Vdash \forall j\left(\operatorname{ent}(\bar{j}), j \varepsilon q_{0} \rightarrow p_{i+1} \neq q_{j+1}\right)$. Therefore $(f \underline{i} x) \lambda j \lambda y(g) j y h \Vdash \perp$. 
ii) We suppose:

$f \nVdash \forall i\left(\operatorname{ent}(i), i \varepsilon p_{0}, \forall j\left(\operatorname{ent}(j), j \varepsilon q_{0} \rightarrow p_{i+1} \neq q_{j+1}\right) \rightarrow \perp\right)$;

$y \nVdash i^{\prime} \varepsilon(p \wedge r)_{0} ; u \Vdash \forall j^{\prime}\left(\operatorname{ent}\left(j^{\prime}\right), j^{\prime} \varepsilon(q \wedge r)_{0} \rightarrow(p \wedge r)_{i^{\prime}+1} \neq(q \wedge r)_{j^{\prime}+1}\right)$.

If we replace $j^{\prime}$ with $2 j^{\prime \prime}$, and then with $2 j^{\prime \prime}+1$, we obtain, by definition of $\wedge$ :

(1) $\quad(u)\left(d_{0}\right) \underline{j}^{\prime \prime} \Vdash j^{\prime \prime} \varepsilon q_{0} \rightarrow(p \wedge r)_{i^{\prime}+1} \neq q_{j^{\prime \prime}+1} ;$

(2) $\quad(u)\left(d_{1}\right) \bar{j}^{\prime \prime} \Vdash j^{\prime \prime} \varepsilon r_{0} \rightarrow(p \wedge r)_{i^{\prime}+1} \neq r_{j^{\prime \prime}+1}$.

Then, there are two cases:

- If $i^{\prime}=2 i^{\prime \prime}$, we have $y \nVdash i^{\prime \prime} \varepsilon p_{0} \quad$ and, by $(1), \quad(u)\left(d_{0}\right) \underline{j}^{\prime \prime} \Vdash j^{\prime \prime} \varepsilon q_{0} \rightarrow p_{i^{\prime \prime}+1} \neq q_{j^{\prime \prime}+1}$. Therefore:

$\lambda j(u)\left(d_{0}\right) j \nVdash \forall j\left(\operatorname{ent}(j), j \varepsilon q_{0} \rightarrow p_{i^{\prime \prime}+1} \neq q_{j+1}\right)$ and it follows that:

$\left(\left((f)\left(d_{2}\right) \underline{i}^{\prime}\right) y\right) \lambda j(u)\left(d_{0}\right) j \Vdash \perp$.

- If $i^{\prime}=2 i^{\prime \prime}+1$, we have $y \nVdash i^{\prime \prime} \varepsilon r_{0}$ and, by $(2),(u)\left(d_{1}\right) j^{\prime \prime} \Vdash j^{\prime \prime} \varepsilon r_{0} \rightarrow r_{i^{\prime \prime}+1} \neq r_{j^{\prime \prime}+1}$.

By making $j^{\prime \prime}=i^{\prime \prime}$, we obtain $(u)\left(d_{1}\right) \underline{i}^{\prime \prime} \Vdash i^{\prime \prime} \varepsilon r_{0} \rightarrow \perp$ and therefore:

(u) $\underline{i}^{\prime} y \Vdash \perp$.

Thus, in both cases, we get: $\quad\left(\left(e \underline{i}^{\prime}\right)(u) \underline{i}^{\prime} y\right)\left(\left((f)\left(d_{2}\right) \underline{i}^{\prime}\right) y\right) \lambda j(u)\left(d_{0}\right) j \Vdash \perp$.

Lemma 6.2.

i) $\theta \Vdash \forall p \forall q(p \subset q, \mathbf{C}[q] \rightarrow \mathbf{C}[p])$ with

$\theta=\lambda f \lambda g \lambda i \lambda i^{\prime} \lambda x \lambda x^{\prime} \lambda u \lambda v \lambda w\left(f i^{\prime} x^{\prime}\right) \lambda j^{\prime} \lambda y^{\prime}(f i x) \lambda j \lambda y(g) j j^{\prime} y y^{\prime} u v w$.

ii) $\| \forall p \forall q \forall r(p \subset q, \mathrm{C}[q \wedge r] \rightarrow \mathrm{C}[p \wedge r])$ in other words $\Vdash \forall p \forall q(p \subset q \rightarrow q \sqsubseteq p)$.

Proof.

i) Let $f \nVdash p \subset q, g \nVdash \mathrm{C}[q]$, that is:

$f \nVdash \forall i\left(\operatorname{ent}(i), i \varepsilon p_{0}, \forall j\left(\operatorname{ent}(j), j \varepsilon q_{0} \rightarrow p_{i+1} \neq q_{j+1}\right) \rightarrow \perp\right)$;

$g \Vdash \forall j \forall j^{\prime}\left(\operatorname{ent}(j), \operatorname{ent}\left(j^{\prime}\right), j \varepsilon q_{0}, j^{\prime} \varepsilon q_{0} \rightarrow E\left[q_{j+1}, q_{j^{\prime}+1}\right]\right)$ with :

$E[x, y] \equiv(x \neq y,(x \triangleleft y) \neq 1,(y \triangleleft x) \neq 1 \rightarrow \perp)$.

Let $x \Vdash i \varepsilon p_{0}, x^{\prime} \Vdash i^{\prime} \varepsilon p_{0}, u \Vdash p_{i+1} \neq p_{i^{\prime}+1}, v \nVdash\left(p_{i+1} \triangleleft p_{i^{\prime}+1}\right) \neq 1, w \nVdash\left(p_{i^{\prime}+1} \triangleleft p_{i+1}\right) \neq 1$.

Let $y \nVdash j \varepsilon q_{0}, y^{\prime} \Vdash j^{\prime} \varepsilon q_{0}$.

We have $g j j^{\prime} y y^{\prime} \| E\left[q_{j+1}, q_{j^{\prime}+1}\right]$; if $p_{i+1}=q_{j+1}$ and $p_{i^{\prime}+1}=q_{j^{\prime}+1}$, then:

$g \underline{j} \underline{j}^{\prime} y y^{\prime} \Vdash \bar{E}\left[p_{i+1}, p_{i^{\prime}+1}\right]$, and therefore $g \underline{j} \underline{j} \underline{j}^{\prime} y y^{\prime} u v w \nVdash \perp$.

Thus, we have $\lambda j \lambda y(g) j j^{\prime} y y^{\prime} u v w \nVdash \operatorname{ent}(\bar{j}), j \varepsilon q_{0} \rightarrow \perp$ if $p_{i+1}=q_{j+1}$ and $p_{i^{\prime}+1}=q_{j^{\prime}+1}$.

Therefore, $\lambda j \lambda y(g) j j^{\prime} y y^{\prime} u v w \nVdash \forall j\left(\operatorname{ent}(j), j \varepsilon q_{0} \rightarrow p_{i+1} \neq q_{j+1}\right)$ if $p_{i^{\prime}+1}=q_{j^{\prime}+1}$, thus:

$(f \underline{i} x) \lambda j \lambda y(g) j j^{\prime} y y^{\prime} u v w \| \perp$ if $p_{i^{\prime}+1}=q_{j^{\prime}+1}$, thus:

$\lambda j^{\prime} \lambda y^{\prime}(f \underline{i} x) \lambda j \lambda y(g) j j^{\prime} y y^{\prime} u v w \Vdash \forall j^{\prime}\left(\operatorname{ent}\left(j^{\prime}\right), j^{\prime} \varepsilon q_{0} \rightarrow p_{i^{\prime}+1} \neq q_{j^{\prime}+1}\right)$. Therefore: $\left(f \underline{i}^{\prime} x^{\prime}\right) \lambda j^{\prime} \lambda y^{\prime}(f \underline{i} x) \lambda j \lambda y(g) j j^{\prime} y y^{\prime} u v w \| \perp$.

ii) Follows immediately from (i) and $\Vdash \forall p \forall q \forall r(p \subset q \rightarrow p \wedge r \subset q \wedge r)$ (lemma 6.1).

The following lemma shows that we can build the algebra $\mathcal{B}$ and the $\mathcal{B}$-model $\mathcal{N}$.

Lemma 6.3. There exist six proof-like terms $\alpha_{0}, \alpha_{1}, \alpha_{2}, \beta_{0}, \beta_{1}, \beta_{2}$ such that:

$\alpha_{0} \Vdash \forall p \forall q \forall r(\mathbf{C}[(p \wedge q) \wedge r] \rightarrow \mathbf{C}[p \wedge(q \wedge r)]) ; \alpha_{1} \Vdash \forall p(\mathbf{C}[p] \rightarrow \mathbf{C}[p \wedge \mathbf{1}]) ;$

$\alpha_{2} \Vdash \forall p \forall q(\mathbf{C}[p \wedge q] \rightarrow \mathbf{C}[q]) ; \beta_{0} \Vdash \forall p(\mathbf{C}[p] \rightarrow \mathbf{C}[p \wedge p]) ; \beta_{1} \Vdash \forall p \forall q(\mathbf{C}[p \wedge q] \rightarrow \mathbf{C}[q \wedge p]) ;$

$\beta_{2} \Vdash \forall p \forall q \forall r \forall s(\mathrm{C}[((p \wedge q) \wedge r) \wedge s] \rightarrow \mathrm{C}[(p \wedge(q \wedge r)) \wedge s])$.

Proof. We only show the first case. By lemma 6.2(i), it suffices to find a proof-like term:

$\theta \Vdash \forall p \forall q \forall r(p \wedge(q \wedge r) \subset(p \wedge q) \wedge r)$. Thus, we suppose:

$y \nVdash i \varepsilon(p \wedge(q \wedge r))_{0} ; u \nVdash \forall j\left(\operatorname{ent}(j), j \varepsilon((p \wedge q) \wedge r)_{0} \rightarrow(p \wedge(q \wedge r))_{i+1} \neq((p \wedge q) \wedge r)_{j+1}\right)$.

There are three cases: 
- $i=2 i^{\prime}$; then, we have $y \nVdash i^{\prime} \varepsilon p_{0}$. We make $j=2 i=4 i^{\prime}$, therefore:

$u \Vdash \operatorname{ent}(2 i), i^{\prime} \varepsilon p_{0} \rightarrow p_{i^{\prime}+1} \neq p_{i^{\prime}+1}$. Thus, we have: $(u)\left(d_{0}\right) \underline{i} y \nVdash \perp$.

- $i=4 i^{\prime}+1$; then, we have $y \nVdash i^{\prime} \varepsilon q_{0}$. We make $j=i+2=4 i^{\prime}+3$, thus:

$u \Vdash \operatorname{ent}(i+2), i^{\prime} \varepsilon q_{0} \rightarrow q_{i^{\prime}+1} \neq q_{i^{\prime}+1}$. Thus, we have: $\left((u)(\sigma)^{2} \underline{i}\right) y \nVdash \perp$.

- $i=4 i^{\prime}+3$; then, we have $y \nVdash i^{\prime} \varepsilon r_{0}$. We make $j=i-3=4 i^{\prime}$, thus:

$u \Vdash \operatorname{ent}(i-3), i^{\prime} \varepsilon r_{0} \rightarrow r_{i^{\prime}+1} \neq r_{i^{\prime}+1}$. Therefore, we have: $\left((u)(\mathrm{p})^{3} \underline{i}\right) y \nVdash \perp$

( $\mathrm{p}$ is the program for the predecessor).

Thus, we put $\theta=\lambda i \lambda y \lambda u\left(\left(\left(e_{4} i\right)(u)\left(d_{0}\right) i y\right)\left((u)(\sigma)^{2} i\right) y\right)\left((u)(\mathrm{p})^{3} i\right) y$, where $e_{4}$ is defined by its execution rule: $e_{4} \star \underline{i} \cdot \xi \cdot \eta \cdot \zeta \cdot \pi \succ \xi \cdot \pi(\operatorname{resp} . \eta \cdot \pi, \zeta \cdot \pi)$ if $i=4 i^{\prime}\left(\right.$ resp. $\left.4 i^{\prime}+1,4 i^{\prime}+3\right)$.

We now show the:

\section{Theorem 6.4.}

The forcing structure $\{\mathbf{C}, \wedge, \mathbf{1}\}$ satisfies the countable downward chain condition in $\mathcal{M}$.

Proof. The hypothesis of the c.d.c. are:

$H_{0} \equiv \forall n \exists p \mathcal{X}(n, p)$;

$H_{1} \equiv \forall n^{\mathrm{ent}} \forall p \forall q\{\mathcal{X}(n, p), \mathcal{X}(n, q) \rightarrow p=q\}$;

$H_{2} \equiv \forall n^{\text {ent }} \forall p \forall q(\mathcal{X}(n, p), \mathcal{X}(s n, q) \rightarrow q \sqsubseteq p) ;$

$H_{3} \equiv \forall n^{\text {ent }} \forall p(\mathcal{X}(n, p) \rightarrow \mathrm{C}[p])$.

Moreover, by theorem 2.1, we have a binary function $f: P^{2} \rightarrow P$ such that:

$\varsigma \Vdash \forall n^{\mathrm{ent}}\left(\exists p \mathcal{X}(n, p) \rightarrow \exists k^{\mathrm{ent}} \mathcal{X}(n, f(n, k))\right)$.

Therefore, by $H_{0}$, we can also use the hypothesis:

$H_{0}^{\prime} \equiv \forall n^{\text {ent }} \exists k^{\text {ent }} \mathcal{X}(n, f(n, k))$.

Let us put $\vec{H}=\left\{H_{0}, H_{0}^{\prime}, H_{1}, H_{2}, H_{3}\right\}$ and $\vec{H}_{*}=\left\{H_{0}, H_{0}^{\prime}, H_{1}, H_{2}\right\}$.

Lemma 6.5. $\vec{H} \vdash \forall p \forall q \forall m^{e n t} \forall n^{e n t}(\mathcal{X}(m, p), \mathcal{X}(n, q) \rightarrow \mathrm{C}[p \wedge q])$.

Proof. We show $\forall m^{\text {int }} \forall n^{\text {int }}(\mathcal{X}(m, p), \mathcal{X}(m+n, q) \rightarrow q \sqsubseteq p)$ by recurrence on $n$.

For $n=0$, this follows from $H_{1}, H_{3}$. For the recurrence step, we use $H_{2}$.

Thus, we have $\forall p \forall q \forall m^{\text {ent }} \forall n^{\text {ent }}(\mathcal{X}(m, p), \mathcal{X}(n, q) \rightarrow p \sqsubseteq q \vee q \sqsubseteq p)$.

From $p \sqsubseteq q$, we deduce $\mathrm{C}[p \wedge p] \rightarrow \mathrm{C}[q \wedge p]$, and the result follows, by $H_{3}$ and $\mathrm{C}[p] \rightarrow \mathrm{C}[p \wedge p]$.

We define the wanted limit $h$ by defining $h_{0}$ and $h_{m+1}$ for each $m \in \mathbb{N}$.

For $m=(i, n, k)$ (that is $(i,(n, k)))$, we put $\left\|m \varepsilon h_{0}\right\|=\left\|\mathcal{X}(n, f(n, k)) \wedge i \varepsilon(f(n, k))_{0}\right\|$;

then $h_{m+1}=(f(n, k))_{i+1}$.

Intuitively, $\mathcal{X}$ defines a sequence of countable sets, and $h$ is the union of these sets.

- Proof of $\vec{H}_{*} \vdash \mathcal{X}(n, p) \rightarrow h \sqsubseteq p$.

By lemma 6.2(ii), it suffices to show $\mathcal{X}(n, p) \rightarrow p \subset h$, that is:

$\mathcal{X}(n, p), i \varepsilon p_{0}, \forall m^{\text {ent }}\left(m \varepsilon h_{0}, \rightarrow h_{m+1} \neq p_{i+1}\right) \rightarrow \perp$, for $n, i \in \mathbb{N}$.

We fix $k \in \mathbb{N}$ and we put $m=(i, n, k)$. By definition of $h$, it suffices to show:

$\mathcal{X}(n, p), i \varepsilon p_{0}, \forall k^{\mathrm{ent}}\left(\mathcal{X}(n, f(n, k)), i \varepsilon(f(n, k))_{0}, \rightarrow(f(n, k))_{i+1} \neq p_{i+1}\right) \rightarrow \perp$.

Now, from $H_{1}, \mathcal{X}(n, p), \mathcal{X}(n, f(n, k))$, we deduce $f(n, k)=p$ and therefore:

$(f(n, k))_{0}=p_{0}$ and $(f(n, k))_{i+1}=p_{i+1}$. Thus, it remains to show:

$\mathcal{X}(n, p), i \varepsilon p_{0}, \forall k^{\mathrm{ent}}\left(\mathcal{X}(n, f(n, k)), i \varepsilon p_{0} \rightarrow p_{i+1} \neq p_{i+1}\right) \rightarrow \perp$.

But this formula follows immediately from $H_{0}^{\prime}$. 
- Proof of $\vec{H} \vdash \mathrm{C}[h]$.

We must show $\mathrm{C}[h]$, that is $m \varepsilon h_{0}, m^{\prime} \varepsilon h_{0} \rightarrow E\left[h_{m+1}, h_{m^{\prime}+1}\right]$. Now, we have:

$m=(i, n, k) ;\left\|m \varepsilon h_{0}\right\|=\left\|\mathcal{X}(n, f(n, k)) \wedge i \varepsilon(f(n, k))_{0}\right\| ; h_{m+1}=(f(n, k))_{i+1} ;$

$m^{\prime}=\left(i^{\prime}, n^{\prime}, k^{\prime}\right) ;\left\|m^{\prime} \varepsilon h_{0}\right\|=\left\|\mathcal{X}\left(n^{\prime}, f\left(n^{\prime}, k^{\prime}\right)\right) \wedge i^{\prime} \varepsilon\left(f\left(n^{\prime}, k^{\prime}\right)\right)_{0}\right\| ; h_{m^{\prime}+1}=\left(f\left(n^{\prime}, k^{\prime}\right)\right)_{i^{\prime}+1}$.

From $\mathcal{X}(n, f(n, k)), \mathcal{X}\left(n^{\prime}, f\left(n^{\prime}, k^{\prime}\right)\right)$, it follows that:

$\mathrm{C}[u]$ with $u=f(n, k) \wedge f\left(n^{\prime}, k^{\prime}\right)$ (lemma 6.5). Therefore, we have:

$\left\|i \varepsilon(f(n, k))_{0}\right\|=\|2 i \varepsilon u\| ;\left\|i^{\prime} \varepsilon\left(f\left(n^{\prime}, k^{\prime}\right)\right)_{0}\right\|=\left\|2 i^{\prime}+1 \varepsilon u\right\|$;

$h_{m+1}=u_{2 i+1} ; h_{m^{\prime}+1}=u_{2 i^{\prime}+2}$.

From C $[u]$, we deduce $E\left[u_{2 i+1}, u_{2 i^{\prime}+2}\right]$, that is $E\left[h_{m+1}, h_{m^{\prime}+1}\right]$.

This completes the proof of theorem 6.4.

The well ordering on $\mathcal{P}(\mathbb{N})$. In the model $\mathcal{N}$, we define the unary predicate:

$\mathcal{G}(x) \equiv \exists p \exists i^{\text {ent }}\left\{\neg \mathcal{J}(p), i \varepsilon p_{0}, x=p_{i+1}\right\}$.

Lemma 6.6. $\| \Vdash \mathcal{G}(x), \mathcal{G}(y) \rightarrow E[x, y]$.

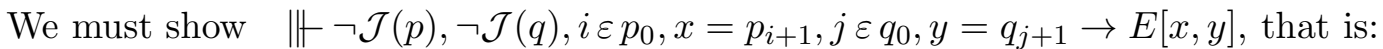

$\| \models \neg \mathcal{J}(p), \neg \mathcal{J}(q), i \varepsilon p_{0}, j \varepsilon q_{0} \rightarrow E\left[p_{i+1}, q_{j+1}\right]$.

By theorem 3.5 (ii) and (iii), we have $\| t \neg \mathcal{J}(p), \neg \mathcal{J}(q) \rightarrow \mathrm{C}[p \wedge q]$.

Therefore, it is sufficient to show that $\| \vdash \mathrm{C}[p \wedge q], i \varepsilon p_{0}, j \varepsilon q_{0} \rightarrow E\left[p_{i+1}, q_{j+1}\right]$.

We show below that we have $I \Vdash \mathrm{C}[p \wedge q], i \varepsilon p_{0}, j \varepsilon q_{0} \rightarrow E\left[p_{i+1}, q_{j+1}\right]$. Since this is a first order formula, this gives the desired result, by theorem 3.3 .

Indeed, we have: $p_{i+1}=(p \wedge q)_{2 i+1} ; q_{j+1}=(p \wedge q)_{2 j+2}$;

$\left\|i \varepsilon p_{0}\right\|=\left\|2 i \varepsilon(p \wedge q)_{0}\right\| ;\left\|j \varepsilon q_{0}\right\|=\left\|2 j+1 \varepsilon(p \wedge q)_{0}\right\|$.

Therefore, it remains to show:

$I \Vdash \mathrm{C}[p \wedge q], 2 i \varepsilon(p \wedge q)_{0}, 2 j+1 \varepsilon(p \wedge q)_{0} \rightarrow E\left[(p \wedge q)_{2 i+1},(p \wedge q)_{2 j+2}\right]$

which is obvious, by definition of $\mathrm{C}[p \wedge q]$.

Lemma 6.6 shows that $\triangleleft$ is a total relation on $\mathcal{G}$. But, moreover, $\triangleleft$ is a well founded relation in $\mathcal{N}$. Therefore, we have:

$\| \vdash \mathcal{G}$ is well ordered by $\triangleleft$.

We define now two functions on $P$ :

- a unary function $\delta: P \rightarrow P$ by putting $\left\|i \varepsilon \delta(p)_{0}\right\|=\left\|i+1 \varepsilon p_{0}\right\| ; \delta(p)_{i+1}=p_{i+2}$.

- a binary function $\phi: P^{2} \rightarrow P$ by putting:

$\left\|0 \varepsilon \phi(p, q)_{0}\right\|=\emptyset ;\left\|i+1 \varepsilon \phi(p, q)_{0}\right\|=\left\|i \varepsilon p_{0}\right\| ;$

$\phi(p, q)_{1}=q ; \phi(p, q)_{i+2}=p_{i+1}$ for every $i \in \mathbb{N}$.

Therefore, we have $\delta(\phi(p, q))=p$ and $\phi(p, q)_{1}=q$ for all $p, q \in P$ and thus:

$I \Vdash \forall p \forall q(\delta(\phi(p, q))=p) ; \quad \mathbf{I} \| \vdash \forall p \forall q(\delta(\phi(p, q))=p)$;

$I \Vdash \forall p \forall q\left(\phi(p, q)_{1}=q\right) ; \quad \mathbf{I} \| \forall \forall p \forall q\left(\phi(p, q)_{1}=q\right)$.

Intuitively, $\delta(p)$ defines the set we obtain by removing $p_{1}$ from the set associated with $p$; $\phi(p, q)$ defines the set we obtain by adding $q$ to the set associated with $p$.

Lemma 6.7. If $p, q \in P$, there exists $q^{\prime} \in P$ such that $\delta\left(q^{\prime}\right)=q$ and $p_{i} \triangleleft q^{\prime}$ for every $i \in \mathbb{N}$.

For each $a \in P$, we have $\delta(\phi(q, a))=q$. But the application $a \mapsto \phi(q, a)$ is obviously injective, since $\phi(q, a)_{1}=a$. Thus, the set $\{\phi(q, a) ; a \in P\}$ is of cardinal $2^{\aleph_{0}}$. Now, by hypothesis on $\triangleleft$, every proper initial segment of $P$, for the well ordering $\triangleleft$, is of cardinal 
$<2^{\aleph_{0}}$. Thus, there exists some $a_{0} \in P$ such that $p_{i} \triangleleft \phi\left(q, a_{0}\right)$ for every $i \in \mathbb{N}$. Then, it suffices to put $q^{\prime}=\phi\left(q, a_{0}\right)$.

Therefore, we can define a binary function $\psi: P^{2} \rightarrow P$ such that we have:

$\delta(\psi(p, q))=q$ and $\left(p_{i} \triangleleft \psi(p, q)\right)=1$ for all $p, q \in P$ and $i \in \mathbb{N}$. Thus, we have:

$I \Vdash \forall p \forall q(\delta(\psi(p, q))=q) ; \mathbf{I} \| \forall \forall p \forall q(\delta(\psi(p, q))=q)$.

$K I \Vdash \forall p \forall q \forall i^{\mathrm{ent}}\left(p_{i} \triangleleft \psi(p, q)\right) ; \mathbf{K I} \| \forall p \forall q \forall i{ }^{\mathrm{ent}}\left(p_{i} \triangleleft \psi(p, q)\right)$.

Lemma 6.8. We have $\|-\forall q \exists x\{\mathcal{G}(x), \delta(x)=q\}$.

Proof. This is written as $\| \Vdash \forall q\left[\forall x \forall p \forall i^{\operatorname{ent}}\left(\delta(x)=q, i \varepsilon p_{0}, x=p_{i+1} \rightarrow \mathcal{J}(p)\right) \rightarrow \perp\right]$ or else:

$\| \vdash \forall q\left[\forall p \forall i^{\text {ent }}\left(i \varepsilon p_{0}, \delta\left(p_{i+1}\right)=q \rightarrow \mathcal{J}(p)\right) \rightarrow \perp\right]$.

By making $i=0$, it is sufficient to show:

$$
\| \vdash \forall q\left[\forall p\left(0 \varepsilon p_{0}, \delta\left(p_{1}\right)=q \rightarrow \mathcal{J}(p)\right) \rightarrow \perp\right] .
$$

By replacing $p$ with $\phi(p, \psi(p, q))$ in (1), we see that it remains to show:

$$
\| \vdash \forall q \neg \forall p \mathcal{J}(\phi(p, \psi(p, q))) \text {. }
$$

Lemma 6.9. $\Vdash \forall p \forall q(\mathrm{C}[p] \rightarrow \mathrm{C}[\phi(p, \psi(p, q))])$.

Proof. We have $\mathrm{C}[r] \equiv \forall i^{\text {ent }} \forall j{ }^{\text {ent }}\left(i \varepsilon r_{0}, j \varepsilon r_{0} \rightarrow E\left[r_{i+1}, r_{j+1}\right]\right)$. Therefore, in order to show that $\nVdash \mathrm{C}[p] \rightarrow \mathrm{C}[r]$, it suffices to show:

(1) $\quad \nVdash \mathrm{C}[p] \rightarrow \forall i^{\mathrm{ent}} \forall j^{\mathrm{ent}}\left(i+1 \varepsilon r_{0}, j+1 \varepsilon r_{0} \rightarrow E\left[r_{i+2}, r_{j+2}\right]\right)$ and

(2) $\quad \nVdash \mathrm{C}[p] \rightarrow \forall j^{\mathrm{ent}}\left(0 \varepsilon r_{0}, j+1 \varepsilon r_{0} \rightarrow E\left[r_{1}, r_{j+2}\right]\right)$.

We apply this remark by putting $r=\phi(p, \psi(p, q))$. Then $(1)$ is written as $\Vdash \mathbf{C}[p] \rightarrow \mathbf{C}[p]$ since $\left\|i+1 \varepsilon r_{0}\right\|=\left\|i \varepsilon p_{0}\right\|$ and $r_{i+2}=p_{i+1}$ and the same for $j$.

Thus, it suffices to show (2), that is:

$$
\Vdash \mathrm{C}[p] \rightarrow \forall j^{\mathrm{ent}}\left(0 \varepsilon \phi(p, \psi(p, q))_{0}, j+1 \varepsilon \phi(p, \psi(p, q))_{0} \rightarrow E\left[\phi(p, \psi(p, q))_{1}, \phi(p, \psi(p, q))_{j+2}\right]\right) .
$$

But, we have $I \Vdash \forall p \forall q\left(0 \varepsilon \phi(p, q)_{0}\right) ; I \Vdash \forall p \forall q\left(j \varepsilon p_{0} \rightarrow j+1 \varepsilon \phi(p, \psi(p, q))_{0}\right)$;

$I \Vdash \forall p \forall q\left(\phi(p, \psi(p, q))_{1}=\psi(p, q)\right) ; I \Vdash \forall p \forall q\left(\phi(p, \psi(p, q))_{j+2}=p_{j+1}\right)$.

Therefore, it remains to show:

$\Vdash \mathrm{C}[p] \rightarrow \forall j^{\mathrm{ent}}\left(j \varepsilon p_{0} \rightarrow E\left[\psi(p, q), p_{j+1}\right]\right)$

which is trivial, since we have $K I \Vdash \forall p \forall q \forall j^{\text {ent }}\left(p_{j+1} \triangleleft \psi(p, q)\right)$.

Lemma 6.10. $\lambda i \lambda x \lambda y((y)(\sigma) i) x \Vdash \forall p \forall q(p \subset \phi(p, q))$.

Proof. This is written as:

$\lambda i \lambda x \lambda y((y)(\sigma) i) x \Vdash \forall i\left(\operatorname{ent}(i), i \varepsilon p_{0}, \forall j\left(\operatorname{ent}(j), j \varepsilon \phi(p, q)_{0} \rightarrow \phi(p, q)_{j+1} \neq p_{i+1}\right) \rightarrow \perp\right)$

which is immediate, by making $j=i+1$.

We have $\| p \subset \phi(p, \psi(p, q))$ (lemma 6.10), and it follows that:

$\nVdash \phi(p, \psi(p, q)) \sqsubseteq p$ (lemma 6.2] $\mathrm{i})$, and thus $\nVdash \mathrm{C}[\phi(p, \psi(p, q))] \rightarrow \mathrm{C}[p \wedge \phi(p, \psi(p, q))]$.

Therefore, by lemma 6.9, we have:

$\Vdash \forall p \forall q(\mathbf{C}[p] \rightarrow \mathbf{C}[p \wedge \phi(p, \psi(p, q))])$. Since this is a first order formula, we have, by theo$\operatorname{rem}[3.3$. $\quad \|+\forall p \forall q(\mathbf{C}[p] \rightarrow \mathbf{C}[p \wedge \phi(p, \psi(p, q))])$

and therefore, by theorem 3.5(ii): $\| \nVdash \forall p \forall q(\neg \mathrm{C}[p \wedge \phi(p, \psi(p, q))] \rightarrow \mathcal{J}(p))$.

Then, we apply theorem 3.6, which gives: $\| \Vdash \forall q \neg \forall p \mathcal{J}(\phi(p, \psi(p, q)))$

which is the desired result. 
Theorem 6.11. The following formulas are realized in $\mathcal{N}$ :

i) There exists a well ordering on the set of individuals.

ii) There exists a well ordering on the power set of $\mathbb{N}$.

Proof.

i) Lemma 6.8 shows that, in $\mathcal{N}$, the function $\delta$ is a surjection from $\mathcal{G}$ onto the set $P$ of individuals. But, we have seen that the formula: " $\mathcal{G}$ is well ordered by $\triangleleft$ " is realized in $\mathcal{N}$.

ii) By theorems 4.4 and 6.4, the following formula is realized in $\mathcal{N}$ : " Every subset of $\mathbb{N}$ is represented by an individual ". Hence the result, by (i).

Theorem 6.11(ii) enables us to transform into a program any proof of a formula of second order arithmetic, which uses the existence of a well ordering on $\mathbb{R}$. The method is the same as the one explained above for the ultrafilter axiom.

\section{REFERENCES}

[1] S. Berardi, M. Bezem, T. Coquand. On the computational content of the axiom of choice. J. Symb. Log. 63 (1998), p. 600-622.

[2] H.B. Curry, R. Feys. Combinatory Logic. North-Holland (1958).

[3] W. Easton. Powers of regular cardinals. Ann. Math. Logic 1 (1970), p. 139-178.

[4] J.Y. Girard. Une extension de l'interprétation fonctionnelle de Gödel à l'analyse. Proc. 2nd Scand. Log. Symp. (North-Holland) (1971) p. 63-92.

[5] T. Griffin. A formula-as-type notion of control. Conf. record 17th A.C.M. Symp. on Principles of Progr. Languages (1990).

[6] S. Grigorieff. Combinatorics on ideals and forcing. Ann. Math. Logic 3(4) (1971), p. 363-394.

[7] W. Howard. The formulas-as-types notion of construction. Essays on combinatory logic, $\lambda$-calculus, and formalism, J.P. Seldin and J.R. Hindley ed., Acad. Press (1980) p. 479-490.

[8] J. M. E. Hyland. The effective topos.

The L.E.J. Brouwer Centenary Symposium (Noordwijkerhout, 1981), 165-216, Stud. Logic Foundations Math., 110, North-Holland, Amsterdam-New York, 1982.

[9] G. Kreisel. On the interpretation of non-finitist proofs $I$. J. Symb. Log. 16 (1951) p. 248-26.

[10] G. Kreisel. On the interpretation of non-finitist proofs II. J. Symb. Log. 17 (1952), p. 43-58.

[11] J.-L. Krivine. Typed lambda-calculus in classical Zermelo-Fraenkel set theory. Arch. Math. Log., 40, 3, p. 189-205 (2001). http://www.pps.jussieu.fr/ krivine/articles/zf_epsi.pdf

[12] J.-L. Krivine. Dependent choice, 'quote' and the clock. Th. Comp. Sc., 308, p. 259-276 (2003). http://hal.archives-ouvertes.fr/hal-00154478 http://www.pps.jussieu.fr/ krivine/articles/quote.pdf

[13] J.-L. Krivine. Realizability in classical logic. In Interactive models of computation and program behaviour. Panoramas et synthèses, Société Mathématique de France, 27 (2009). http://hal.archives-ouvertes.fr/hal-00154500 Updated version at: http://www.pps.jussieu.fr/ krivine/articles/Luminy04.pdf

[14] J.-L. Krivine. Realizability : a machine for Analysis and set theory. Geocal'06 (fevrier 2006 - Marseille); Mathlogaps'07 (juin 2007 - Aussois). http://cel.archives-ouvertes.fr/cel-00154509 Updated version at: http://www.pps.jussieu.fr/ krivine/articles/Mathlog07.pdf 
[15] J.-L. Krivine. Structures de réalisabilité, RAM et ultrafiltre sur $\mathbb{N}$. (2008) http://hal.archives-ouvertes.fr/hal-00321410 http://www.pps.jussieu.fr/ krivine/articles/Ultrafiltre.pdf

$* * * * * * * * * * *$

This work is licensed under the Creative Commons Attribution-NoDerivs License. To view a copy of this license, visit http://creativecommons.org/licenses/by-nd/2.0/ or send a letter to Creative Commons, 171 Second St, Suite 300, San Francisco, CA 94105, USA, or Eisenacher Strasse 2, 10777 Berlin, Germany 